\title{
6-Substituted Hexamethylene Amiloride (HMA) Derivatives as Potent and Selective Inhibitors of the Human Urokinase Plasminogen Activator for Use in Cancer
}

\author{
Benjamin J. Buckley ${ }^{\dagger, \ddagger}$, Ashraf Aboelela ${ }^{\dagger, \ddagger}$, Elahe Minaei ${ }^{\dagger, \ddagger}$, Longguang X. Jiang ${ }^{\|}$Zhihong \\ $\mathrm{Xu}^{\perp}$, Umar Ali ${ }^{\dagger} \ddagger$, Karen Fildes ${ }^{\ddagger}, \S$, Chen-Yi Cheung ${ }^{\#}$, Simon M. Cook ${ }^{\ddagger}$, Darren C. Johnson ${ }^{\nabla}$, \\ Daniel A. Bachovchin $\nabla, \bigcirc$, Gregory M. Cook", Minoti Apte ${ }^{\perp}$, Mingdong Huang", Marie \\ Ranson ${ }^{*},+\ddagger$, and Michael J. Kelso ${ }^{*}, \dagger, \ddagger$
}

†Molecular Horizons and School of Chemistry \& Molecular Bioscience, University of Wollongong, Wollongong, NSW 2522, Australia $\neq$ Illawarra Health \& Medical Research Institute, Wollongong, NSW 2522, Australia §Graduate School of Medicine, University of Wollongong, Wollongong, NSW 2522, Australia "National Joint Biomedical Engineering Research Centre on Photodynamic Technologies, Fuzhou University, Fujian 350116, China ${ }^{\perp}$ Pancreatic Research Group, South Western Sydney Clinical School, University of New South Wales, and Ingham Institute for Applied Medical Research, Liverpool, NSW 2170, Australia \#Department of Microbiology and Immunology, University of Otago, Otago 9016, New Zealand ${ }^{\nabla}$ Tri-Institutional PhD Program in Chemical Biology, Memorial Sloan Kettering Cancer Center, New York, New York 10065, United States ${ }^{\circ}$ Chemical Biology Program, Memorial Sloan Kettering Cancer Center, New York, New York 10065, United States

\section{Abstract}

\begin{abstract}
Metastasis is the cause of death in the majority ( $~ 90 \%)$ of malignant cancers. The oral potassiumsparing diuretic amiloride and its 5-substituted derivative 5-N,N-(hexamethylene)amiloride (HMA) reportedly show robust antitumor/ metastasis effects in multiple in vitro and animal models. These effects are likely due, at least in part, to inhibition of the urokinase plasminogen activator (uPA), a key protease determinant of cell invasiveness and metastasis. This study reports the discovery of 6-substituted HMA analogs that show nanomolar potency against uPA, high selectivity over related trypsin-like serine proteases, and minimal inhibitory effects against epithelial sodium channels (ENaC), the diuretic and antikaliuretic target of amiloride. Reductions in lung metastases were demonstrated for two analogs in a late-stage experimental mouse metastasis model, and one analog completely inhibited formation of liver metastases in an
\end{abstract}

\footnotetext{
*Corresponding Authors M.R.: mranson@uow.edu.au; phone, +61 24221 3291. M.J.K.: mkelso@ uow.edu.au; phone, +61 24221 5085.

The authors declare no competing financial interest.

ASSOCIATED CONTENT

Supporting Information

The Supporting Information is available free of charge on the ACS Publications website at DOI: 10.1021/acs.jmedchem.8b00838. X-ray crystallography refinement data and statistics, HT-1080 uPA and uPAR expression data, experimental late-stage metastasis model clinical score sheet (PDF) Molecular formula strings of all compounds (CSV)
} 
orthotopic xenograft mouse model of pancreatic cancer. The results support further evaluation of 6-substituted HMA derivatives as uPA-targeting anticancer drugs.

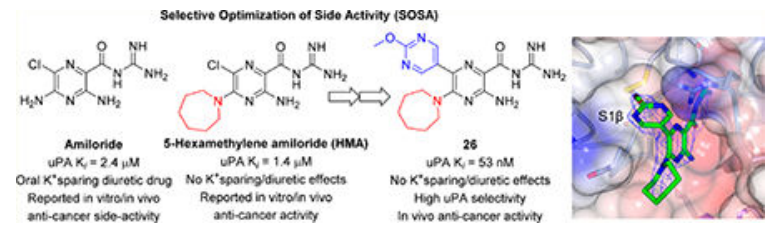

\section{INTRODUCTION}

The urokinase plasminogen activator (UPA) is a trypsin-like serine protease (TLSP) that has been implicated in the invasive spread and metastatic dissemination of transformed cells in several aggressive cancers, including triple-negative breast, pancreatic, gastric, colorectal, and prostate cancers. ${ }^{1-5}$ Clinically, uPA expression provides the highest level of evidence (level 1) ${ }^{6}$ for predicting poor patient prognosis in breast cancer and it is one of the strongest predictors of progression-free survival and overall survival. ${ }^{7-9}$

uPA binds to cell surfaces via a specific interaction with its cognate receptor urokinase plasminogen activator receptor (uPAR), which is expressed on cancer cells and the supporting stroma, often at the invasive front of tumors. ${ }^{10-12}$ uPAR-bound UPA activates colocalized plasminogen to yield the broad spectrum TLSP plasmin, ${ }^{13}$ a key mediator of directed pericellular proteolysis that activates multiple downstream proteases (e.g., matrix metalloproteinases and cathepsins), ultimately leading to degradation of the extracellular matrix and basement membrane components that confine primary tumors. ${ }^{3,14,15}$ The uPAinitiated proteolytic cascade is thus a key determinant of local invasion and metastatic spread via the lymphatic and circulatory systems. ${ }^{16,17}$

uPA attracted much interest in the 1990s and early 2000s as an anticancer target in academic and industry laboratories (reviewed by Ngo et al. ${ }^{18}$ and Tyndall et al. ${ }^{19}$ ). For example, efforts from Abbott Laboratories led to highly potent naphthamidine-based inhibitors, e.g., 1. ${ }^{20-23} \mathrm{X}$-ray cocrystal structures of these and other inhibitors uncovered a novel subsite, termed $\mathrm{S} 1 \beta$, adjacent to the $\mathrm{S} 1$ binding pocket in uPA. Targeting of this site afforded potency gains in a variety of arylamidine-based inhibitors. ${ }^{20,24}$ The poor drug properties of arylamidines ultimately proved to be a major stumbling block, however, and compounds were not advanced to the clinic. ${ }^{25}$ Astex Therapeutics were able to repurpose the oral antiarrhythmic drug mexiletine into a potent, orally active non-amidine-containing uPA inhibitor $2,{ }^{26}$ but no clinical studies have been reported to date.

3a and its oral hydroxyamidine prodrug $3 b$ (Heidelberg Pharma AG; formerly WILEX AG) are, to the best of our knowledge, the only uPA inhibitors to have undergone clinical evaluation in cancer. ${ }^{27}$ Phase II studies of oral $\mathbf{3 b}$ in combination with gemcitabine showed a $17 \%$ increase in 1-year survival in patients with locally advanced, nonresectable pancreatic cancers compared to patients treated with gemcitabine alone. ${ }^{28}$ In a separate phase II study, patients with HER2-negative metastatic breast cancers who had received adjuvant chemotherapy following primary diagnosis and were subsequently treated with $\mathbf{3 b}$ in 
combination with capecitabine showed an increase in median progression-free survival ( 8.3 months) compared to patients receiving capecitabine alone (4.3 months). ${ }^{29}$

Amiloride 4 is an oral potassium-sparing diuretic that has been used for over 4 decades in the treatment of hypokalemia, hypertension, and cirrhosis. ${ }^{30,31}$ The diuretic and antikaliuretic properties of amiloride arise through inhibition of renal epithelial sodium channels (ENaCs). ${ }^{32,33}$ Several groups have reported that the compound also shows anticancer side-activities in multiple in vitro and rodent models (reviewed by Matthews et al.). ${ }^{16}$ In one study, high doses of amiloride ( $200 \mathrm{mg} / \mathrm{kg}$ in drinking water ad libitum) caused complete remission in a prostate xenograft mouse model. ${ }^{34}$ It has been suggested that the anticancer effects of amiloride observed at the high doses used in this and other animal studies likely arise, at least in part, from inhibition of uPA proteolytic activity $\left(\mathrm{IC}_{50}=7 \mu \mathrm{M}\right)$. 35 Amiloride is unsuitable for use clinically as an anticancer drug, however, due to its low maximum daily dose ceiling ( $20 \mathrm{mg} / \mathrm{day}$ ), where overdose risks hyperkalemia and associated cardiac events. ${ }^{36}$ Nevertheless, the demonstrable in vivo anticancer side-activities of amiloride, combined with excellent drug-like properties (inferred from decades of oral clinical use) and low micromolar potency against uPA, suggest it as an attractive starting scaffold for repurposing using the selective optimization of side-activity (SOSA) approach ${ }^{37}$ into a high potency uPA inhibitor for treating uPA-driven cancers. ${ }^{16}$ Moreover, the reduced basicity of the acyl guanidine group relative to arylamidines potentially offers a solution to the poor drug properties of earlier inhibitors. ${ }^{25}$ We propose that effective dosing of an analog suitably optimized for high uPA potency that lacks the diuretic and antikaliuretic effects of amiloride may safely and effectively recapitulate in humans the anticancer activities observed with high doses of amiloride in animals.

Our preliminary structure-activity exploration of amiloride analogs as uPA inhibitors focused on the effects of varying substituents at the $5-\mathrm{NH}_{2}$ group. ${ }^{38}$ Relatively flat SAR trends were observed, with multiple analogs carrying diverse 5-substituents returning $\mathrm{IC}_{50}$ values in the range $1-50 \mu \mathrm{M}$. Large increases in potency relative to amiloride were not realized, with the most active compound (5- $N, N^{\prime}$-dimethylethylenediamine amiloride) showing only a $\sim 2$-fold increase $\left(\mathrm{IC}_{50}=3 \mu \mathrm{M}\right)$. A key finding, however, was that 5-N,N(hexamethylene)amiloride 5 (HMA, Figure 1) retained potency against uPA ( $\mathrm{IC}_{50}=6 \mu \mathrm{M}$ ).

HMA has a long history of use as a pharmacological tool ${ }^{39}$ and, like amiloride, reportedly shows in vitro and in vivo anticancer activities. ${ }^{40-45}$ For example, HMA has been shown to delay primary tumor growth and increase thermosensitivity of SCK mammary carcinoma cells in a mouse allograft model, ${ }^{40,46}$ and it was recently shown to suppress sorafenibresistant engraftment of FLT3-ITD+ acute myeloid leukemia primary cells in mice, with total suppression observed in combination with sorafenib. ${ }^{43}$ Importantly, alkyl substitution of the 5- $\mathrm{NH}_{2}$ group of amiloride (as in HMA) appears to reduce inhibitory effects against ENaCs. ${ }^{46,47}$ On the basis of this, HMA 5 was selected as the starting point for SOSA optimization.

There have been many structure-activity studies exploring the effects of substitution at various positions around the pyrazine core of amiloride, ${ }^{46}$ but there is a surprising lack of reports on analogs carrying variations at the 6-position of amiloride, HMA, or any other 5- 
substituted amilorides. ${ }^{48}$ This study reports the synthesis, uPA structure-activity relationships, and in vivo anticancer properties of HMA analogs carrying (hetero)aryl substituents at the 6-position.

\section{RESULTS AND DISCUSSION}

\section{Chemistry.}

The reported X-ray cocrystal structure of amiloride bound to $\mathrm{uPA}^{49}$ indicated that the 6-Cl group projects toward the $\mathrm{S} 1 \beta$ subsite, suggesting that other groups attached here might better occupy the site and increase potency. A diverse series of 6-(hetero)aryl substituted HMA analogs was produced from the common 5- $N, N$-(hexamethylene)amiloride methyl ester intermediate 7 (Scheme 1). Ester 7 was obtained via nucleophilic aromatic substitution of the commercially available 3-amino-5,6-dichloropyrazine-2-methyl ester 6 with hexamethyleneimine. The reported method for preparing $7,{ }^{38}$ which involves heating 6 with hexamethyleneimine in DMF at $100{ }^{\circ} \mathrm{C}$ for $1 \mathrm{~h}$, requires multiple column chromatography steps to obtain pure 7 (65\% yield). We found that when the reaction was instead performed at reflux in 2-propanol in the presence of $N, N$-diisopropylethylamine (DIPEA), an excellent yield of $7(86 \%)$ was obtained after only $2 \mathrm{~h}$. The improved procedure could be performed on multigram scale in an open flask with the pure $\mathbf{7}$ crystallizing from the reaction mixture and isolated by simple filtration.

Direct attachment of (hetero)aryl substituents to the 6-position of 7 was achieved using standard Suzuki-Miyaura cross-coupling conditions with commercial arylboronic acids (or pinacol boronates), $\mathrm{Pd}\left(\mathrm{PPh}_{3}\right)_{4}$, and $\mathrm{K}_{2} \mathrm{CO}_{3}$ in toluene/MeOH (4:1) at reflux. A total of twenty-three 6-(hetero)aryl methyl esters 9a-32a were produced in yields ranging from 34 to $93 \%$. The methyl esters were converted to their respective acylguanidines 9-32 in 2-92\% by treatment with guanidine. Low guanidinylation yields were initially obtained using the reported method ${ }^{38}$ leading us to search for an improved procedure. Treating the methyl esters in DMF with a $2 \mathrm{M}$ stock solution of guanidine in $\mathrm{MeOH}$ was found to give much higher yields, with most reactions proceeding to completion at room temperature and no major side products forming. The revised procedure was simple to carry out and allowed multiple guanidinylation reactions to be performed in parallel, and the $2 \mathrm{M}$ guanidine solution could be stored under $\mathrm{N}_{2}$ in a refrigerator for up to 2 weeks. ${ }^{50}$

6-(2-(Alkylamino)pyrimidin-5-yl) HMA analogs 33-35 (Scheme 2) were obtained from 6(2-chloropyrimidin-5-yl) methyl ester intermediate 8. Compound 8 was synthesized in $64 \%$ yield by Suzuki-Miyaura coupling of 7 with (2-chloropyrimidin-5-yl)boronic acid. Nucleophilic aromatic substitution of the 2-chloro group with the requisite amines yielded esters 33a-35a in $83-87 \%$ yields, which were converted to acyl guanidines 33-35 in $85-89 \%$ yields using the new guanidinylation procedure.

Previous reports describing napthamidine-based inhibitors ${ }^{20,21}$ identified that the 2aminopyrimidine group shows favorable binding in the uPA $\mathrm{S}_{1} \beta$ pocket. On the basis of this, 2 -aminopyrimidinyl derivative $\mathbf{4 1}$ was synthesized using the route outlined in Scheme 3. The $3-\mathrm{NH}_{2}$ group of methyl ester 7 was di-Boc protected using Boc-anhydride to give 36 in $85 \%$ yield. Halogen exchange from the 6-chloro group to 6-bromo in preparation for Buchwald 
-Hartwig amination was carried out over two steps. Hydrodechlorination using 1 atm of hydrogen in the presence of $\mathrm{Pd} / \mathrm{C}$ and $\mathrm{MgO}$ was successful, although it was accompanied by loss of one Boc-group giving mono-Boc derivative $\mathbf{3 7}$ in $92 \%$ yield. Introduction of the 6bromo substituent using $N$-bromosuccinimide (NBS) gave $\mathbf{3 8}$ ( $60 \%$ yield), and Buchwald -Hartwig amination of $\mathbf{3 8}$ with 2-aminopyrimidine using $\mathrm{Pd}_{2}(\mathrm{dba})_{3} /$ Xantphos afforded the 6-substituted pyrazine methyl ester $\mathbf{3 9}$ in 30\% yield. Removal of the remaining Boc-group with trifluoroacetic acid (98\% yield) followed by guanidinylation delivered $\mathbf{4 1}$ (35\% yield).

\section{Inhibition of uPA and Structure-Activity Relationships.}

Inhibition of catalytically active, low molecular weight human uPA by 9-35 and 41 was determined using a fluorometric enzyme activity assay. ${ }^{51}$ Concentration-response curves for representative analogs $\mathbf{1 8}$ and $\mathbf{2 7}$ are provided in Figure 2, and calculated $K_{\mathrm{i}}$ values are presented in Table 1.

Amiloride 4 and HMA 5 returned $K_{\mathrm{i}}$ values of $2433 \mathrm{nM}$ and $1356 \mathrm{nM}$, respectively, under the assay conditions. Replacing the 6-Cl group of HMA with a phenyl substituent 9 led to $\sim 3.7$-fold decrease in potency. Substitution of the phenyl ring at the para-position (10 and 11) did not improve potency, while conversion to the 3,4-methylenedioxy derivative $\mathbf{1 2}$ provided a slight increase relative to HMA $\left(K_{\mathrm{i}}=934 \mathrm{nM}\right)$. Appending an isoquinolyl group $\mathbf{1 3}$ reduced activity nearly 3 -fold relative to HMA. $N$-Methylpyrazole derivative $\mathbf{1 4}$ showed a $\sim 2.7$-fold increase $\left(K_{\mathrm{i}}=508 \mathrm{nM}\right)$. Extension of the $N$-methyl group of 14 to $N$ ethylmorpholino 15 had only a minor effect $\left(K_{\mathrm{i}}=658 \mathrm{nM}\right)$. Surprisingly, while 2-indolyl 16 and 2-benzothiophenyl substituents $\mathbf{1 7}$ showed reduced activity, the single atom change to 2benzofuranyl derivative 18 afforded a significant boost in potency $\left(K_{\mathrm{i}}=183 \mathrm{nM}\right)$, which increased 2-fold upon addition of a fluorine atom at the benzofuran 4-position (19, $K_{\mathrm{i}}=88$ $\mathrm{nM})$. The preference for oxygen in the heteroaryl ring was also seen with the 2- and 3furanyl derivatives $\mathbf{2 2}$ and $\mathbf{2 3}$, respectively, which both showed nM potency, whereas 2- and 3-thiophene derivatives $\mathbf{2 0}$ and $\mathbf{2 1}$ were micromolar inhibitors.

2-Fluoropyridine substitution ( $\left.24 K_{\mathrm{i}}=1778 \mathrm{nM}\right)$ afforded similar activity to HMA 5, while addition of a 5-pyrimidinyl group 25 ( $K_{\mathrm{i}}=108 \mathrm{nM}$ ) gave a $\sim 12.6$-fold increase. Addition of a methoxy group to the pyrimidine 2-position yielded the first sub-100 nM inhibitor $\mathbf{2 6}\left(K_{\mathrm{i}}=\right.$ $53 \mathrm{nM})$, and adding a second methoxy group to the pyrimidine 4-position $27\left(K_{\mathrm{i}}=42 \mathrm{nM}\right)$ gave a further increase. The favorable binding of the pyrimidine group was evident from the significant losses in potency observed with dimethoxypyridine $\mathbf{2 8}$ and dimethoxyphenyl derivatives $\mathbf{2 9}$ and $\mathbf{3 0}$. Loss of potency with the dimethoxypyridazine $\mathbf{3 1}$ was less dramatic $\left(K_{\mathrm{i}}=315 \mathrm{nM}\right)$. An additional 2-fold gain in potency was achieved upon addition of an amine to the pyrimidine 2-position, yielding the most potent inhibitor in the study $\left(32 K_{\mathrm{i}}=21 \mathrm{nM}\right)$. Further gains in potency were not obtained through $N$-alkyl substitution, with $N$-methyl 33, $\mathrm{N}$-isopropyl 34, and $\mathrm{N}$-hydroxyethyl $\mathbf{3 5}$ derivatives showing activity similar to $\mathbf{2 6}$. The 2aminopyrimidine derivative $\mathbf{4 1}$ obtained using Buchwald-Hartwig chemistry showed lower potency $\left(K_{\mathrm{i}}=228 \mathrm{nM}\right)$. 


\section{X-ray Crystallography.}

An X-ray cocrystal structure was obtained for HMA 5 bound to the catalytic domain of human uPA (Figure 3). The binding orientation and interactions observed in the HMA:uPA structure mirrored those previously observed with amiloride $4 .{ }^{49}$ The key salt bridge interaction was present between the acylguanidine of HMA and the Asp189 side chain carboxylate located at the base of the $\mathrm{S} 1$ binding pocket. H-bonds were present between the backbone carbonyl of Gly219 and the terminal NH and amide NH groups of the acyl guanidine. The second terminal guanidine- $\mathrm{NH}$ formed an $\mathrm{H}$-bond to the side chain hydroxyl of Ser190, and the exocyclic $\mathrm{NH}_{2}$ group at $\mathrm{C} 3$ formed an $\mathrm{H}$-bond to the side chain hydroxyl of Ser195. A bridging H-bond network was observed between a surface-bound water molecule, the acyl guanidine carbonyl oxygen, Ser190 side chain hydroxyl, and a guanidine $\mathrm{NH}$ group. A sulfate ion from the crystallization buffer was observed in the oxyanion hole of both structures.

X-ray cocrystal structures were similarly obtained for benzofuran $(\mathbf{1 8}, \mathbf{1 9})$, furan $(\mathbf{2 2}, \mathbf{2 3})$, pyrimidine 25, 2-methoxypyrimidine 26, 2,4-dimethoxypyrimidine 27 , and $N$ methylpyrazole 14 analogs (Figures 4 and 5. X-ray refinement statistics are provided in Supporting Information, Table S1). Hbonding networks reminiscent of those seen in the HMA:uPA structure were observed in all complexes. The seven-membered hexamethylene ring appeared to sample multiple conformations, as evidenced by low electron density in all structures. Replacement of the 6- $\mathrm{Cl}$ group with (hetero)aryl substituents did not significantly alter the binding positions or orientation of the pyrazine core or acylguanidines relative to HMA 5. The 6-substituent occupied S1 $\beta$ in all cases, and sulfate ion occupied the oxyanion hole in some complexes.

The structure obtained with 2-furanyl analog 22 (Figure 4 a) revealed that the $\sim 8.7$-fold increase in potency relative to HMA 5 may arise from new H-bonds formed between the furanyl O, a bridging water molecule, and the amide $\mathrm{NH}$ of Gly219. Favorable van der Waals contacts between the furan ring and Gly219, the Cys191-Cys220 disulfide, Ser146 backbone carbonyl, and the side chain $\mathrm{C} \beta$ and $\mathrm{C} \gamma$ of $\mathrm{Gln} 192$ were also apparent. Changing to the 3-furanyl substituent $\mathbf{2 3}$ had little effect on uPA activity despite the furanyl oxygen no longer making the water-bridged H-bond interaction with Gly219 (Figure 4b). Flexibility of the $\operatorname{Arg} 217$ side chain was noted in this structure, where it had shifted toward $\mathrm{S} 1 \beta$ to participate in the Gly219-bridging water H-bond network. Relative to 22, the plane of the furan ring in $\mathbf{2 3}$ was tilted $11^{\circ}$, a pose that promoted van der Waals interactions with Cys220. Interactions with $\mathrm{S} 1 \beta$ residues Cys191-Cys220, Ser146, and the Gln192 side chain were preserved, and there appeared to be a dipolar interaction between the side chain carbonyl of Gln192 and the polarized furan 2-CH group.

Ring fusion to give benzofuran 18 led to a slight decrease in potency $\left(K_{\mathrm{i}}=183 \mathrm{nM}\right)$ relative to 22 despite this group extending further into $S 1 \beta$ and almost completely occupying the subsite (Figure $4 \mathrm{c}$ ). The plane of the benzofuran ring was essentially perpendicular to the pyrazine core, but the ring oxygen had flipped $180^{\circ}$ relative to the furanyl oxygen of $\mathbf{2 2}$. This orientation allowed the benzofuran 7-CH group to fill a small, hydrophobic dimple formed by Lys143, Gln192, and Ser146. Additional hydrophobic contacts were seen 
between the benzofuran 3- and 4-position $\mathrm{CH}$ groups and Gly219. Similar to what was observed in the HMA 5 and $\mathbf{2 2}$ structures, the side chain of Arg217 adopted a conformation well away from $S 1 \beta$. The structure with 19 suggested that the 2-fold increase in potency obtained after fluorine substitution at the benzofuran 4-position $\left(19 K_{\mathrm{i}}=88 \mathrm{nM}\right)$ arose from favorable interactions between the 4-fluoro group and the side chain guanidine of $\operatorname{Arg} 217$ (possibly an H-bond), which had shifted back toward S1 $\beta$ in this complex. The interaction with $\operatorname{Arg} 217$ also appeared to cause a slight twisting about the benzofuran-pyrazine axis away from perpendicular, with the $78^{\circ}$ dihedral angle in 18 reducing to $68^{\circ}$ in 19 .

The 5-pyrimidine substituent of $\mathbf{2 5}$ was found to partially occupy S1 $\beta$ (Figure 5a), making van der Waals interactions with the Cys191-Cys220 disulfide, Ser146, and Gln192 residues. A dipole interaction between the polarized 4-CH group of the pyrimidine and the Gln192 side chain carbonyl appeared to be present, along with an $\mathrm{H}$-bond between the amide $\mathrm{NH}$ of Gly219 and the lone pair of electrons on a pyrimidine nitrogen. The polarized 6- $\mathrm{CH}$ on the pyrimidine appeared to interact with the Gly219 carbonyl group.

Addition of a methoxy group to the pyrimidine 2-position 26 led to a $\sim 2$-fold increase in potency, but it did not appear to make any specific interactions with the protein, instead orienting toward bulk solvent (Figure 5b). The side chain of Arg217 had flipped back toward $\mathrm{S} 1 \beta$ driven by a polar interaction with the pyrimidine nitrogen (possibly an H-bond).

Introduction of a second methoxy substituent onto the pyrimidine ring $\mathbf{2 7}$ resulted in a small increase in potency relative to $\mathbf{2 6}$. The pyrimidine and pyrazine rings were almost perpendicular (dihedral angle $80^{\circ}$ ), allowing for increased occupancy of $S 1 \beta$ and projection of a methoxy group toward the small hydrophobic dimple formed by Lys143, Gln192, and Ser146 (Figure 5c). The second methoxy substituent was projected toward bulk solvent. In contrast to $\mathbf{2 5}$ and $\mathbf{2 6}$, the Arg217 side chain was positioned well away from $\mathrm{S} 1 \beta$, apparently to avoid a steric clash with the pyrimidine $2-\mathrm{OCH}_{3}$ group of 27 .

The $N$-methylpyrazole substituent of $\mathbf{1 4}$ adopted a coplanar arrangement with the pyrazine reducing its penetration into $\mathrm{S} 1 \beta$ (Figure $5 \mathrm{~d}$ ). Interactions were observed between the pyrazole 3-CH and 5-CH groups and the backbone of Gly219 and the side chain of Gln192, respectively. The surface water molecule bound to Gly219 in the furanyl structures $\mathbf{2 2}$ and $\mathbf{2 3}$ was observed but did not appear to interact with the pyrazole.

\section{Inhibition of Trypsin-like Serine Proteases.}

HMA 5 and 6-substituted analogs 18, 19, and 26 were screened against a panel of trypsinlike serine proteases (TLSPs) using chromogenic assays (Table 2). In agreement with earlier reports showing that occupancy of uPA's $\mathrm{S} 1 \beta$ subsite confers high selectivity over other TLSPs, ${ }^{20,24,53}$ each of the compounds showed $20-100+$ fold selectivity for uPA.

To further probe uPA selectivity, superfamily wide screening against 85 serine hydrolases was performed in situ using the recently described EnPlex activity-based protein profiling (ABPP) assay. ${ }^{54}$ In this approach, individual serine hydrolases are conjugated to differently colored Luminex beads and the ability of inhibitory compounds to outcompete a biotinylated serine-reactive/streptavidin-phycoerythrin probe at the active site of each enzyme provides a 
rapid and semiquantitative readout of enzyme inhibition. Results from the Enplex screen with amiloride 4, HMA 5, and 6-substituted analogs 18, 25, and 26 are shown in Figure 6.

Amiloride showed slight activity against only a few serine hydrolases (C1r, C1s, DDHD1, factor VIIa, and LACTB), consistent with its reported high selectivity for uPA. ${ }^{35}$ HMA 5 showed activity against the highly homologous $\mathrm{S} 28$ protease family members dipeptidyl peptidase 2 (DPP7) and prolylcarboxypeptidase (PRCP). ${ }^{55}$ Surprisingly, inhibitory activity against uPA was not observed for HMA under these assay conditions. 2-Benzofuranyl analog 18 showed the least selectivity among the tested compounds, inhibiting plasma kallikrein (KLKb1) and trypsin. 5-Pyrimidinyl analog 25 showed remarkable selectivity for uPA, with slight inhibition of trypsin being the only off-target TLSP activity observed. 2Methoxypyrimidinyl analog $\mathbf{2 6}$ showed slight activity against C1s, plasma kallikrein, plasmin, prolylcarboxy peptidase, trypsin, and transmembrane protease serine 11D (TMPRSS11d).

\section{Human Cell Cytotoxicity.}

HMA 5 and representative analogs $18,19,25$, and 26 were tested for cytotoxicity against four human cell lines (Table 3). HMA $\mathbf{5}$ showed toxicity in the low micromolar range against all cell lines, with HEK-293 embryonic kidney and HT-1080 fibrosarcoma cells being most sensitive. In general, the cell lines were more sensitive to 18, suggesting the benzofuran group may contribute to cytotoxic effects. MDA-MB-231 cells were $\sim 3$-fold more sensitive to the 4-fluorinated benzofuran 19, but the compound was less toxic than $\mathbf{1 8}$ toward the other three cell types. Cytotoxicity was not a general feature of 6-substituted HMA analogs since pyrimidine analogs $\mathbf{2 5}$ and $\mathbf{2 6}$ showed much lower activity against all cells. The lower $\log D_{7.4}$ of the pyrimidines (i.e., 2.8 and 3.4, respectively) relative to benzofuran 18 (i.e., $\log$ $\left.D_{7.4}=5.1\right)$ and HMA ( $\left.\log D_{7.4}=3.8\right)$ suggests lipophilicity may be a contributor to cytotoxicity.

\section{Off-Target Activity against GPCRs.}

Amiloride and related analogs are known to bind to G-protein-coupled receptors (GPCRs). 56,57 For example, amiloride is a known antagonist of the adenosine $2 \mathrm{~A}$ receptor $\left(\mathrm{A}_{2 \mathrm{~A}}\right) .{ }^{58-60}$ Recent work describing the effects of substitution at the amiloride 5-position on $\mathrm{A}_{2 \mathrm{~A}}$ antagonism identified analogs with increased potency, including HMA $5 .{ }^{61}$ In the current study, a representative selection of 6-substituted HMA analogs were evaluated alongside HMA 5 for $\mathrm{A}_{2 \mathrm{~A}}$ antagonism using a radiolabeled ligand displacement assay (Figure 7). In all cases, the presence of the 6-substituent diminished antagonist effects relative to HMA, with the majority of compounds showing poor $\mathrm{A}_{2 \mathrm{~A}}$ binding.

Development of active site-directed uPA inhibitors has been complicated by the observation that compounds optimized for high affinity against human uPA often show reduced potency against the homologous mouse enzyme. ${ }^{62}$ This species selectivity can confound interpretation of data from mouse models as the decreased affinity for mouse uPA may lead to an underestimation of compound efficacy. To explore this issue in the 6-substituted HMA class, we measured the activities of $\mathbf{4}, \mathbf{5}, \mathbf{1 8}$, and $\mathbf{2 6}$ against mouse uPA (Table 4). 
Amiloride 4 was essentially equipotent against mouse and human uPA. Addition of the 5hexamethylene ring in $\mathbf{5}$ decreased affinity for mouse uPA by almost 7-fold, while compounds $\mathbf{1 8}$ and $\mathbf{2 6}$ showed larger (10- to 30-fold) reductions. These findings align with reported trends for compounds bearing substituents that target uPA's S1 $\beta$ subsite. ${ }^{62}$ The lower potency against mouse uPA was a key consideration in dose selection for in vivo studies with $\mathbf{1 8}$ and $\mathbf{2 6}$ (see below).

\section{Diuretic and Antikaliuretic Effects.}

The potassium-sparing activity of amiloride arises from inhibition of epithelial sodium channels $(\mathrm{ENaCs})$ in the kidneys, leading to reduced urinary excretion of $\mathrm{K}^{+} .32$ In cases of overdose, the potassium-sparing effects can lead to hyperkalemia and cardiac arrhythmias. ${ }^{63}$ As such, removal of ENaC activity is essential for development of a 6-substituted HMA analog suitable for use in cancer. Early work from the Cragoe group at Merck established that alkyl substitution of the 5- $\mathrm{NH}_{2}$ group of amiloride reduces $\mathrm{ENaC}$ activity ${ }^{47}$ and showed that HMA produces $>80$-fold lower inhibition than amiloride in a deoxycorticosterone acetate (DOCA) induced $\mathrm{K}^{+}$excretion model in adrenalectomized rats. ${ }^{47} 6$-Substituted HMA analogs were thus expected to show similarly low activity against ENaCs. To confirm this, a representative selection of analogs was tested against recombinant HEK-293 cells that overexpress the $a, \beta$, and $\gamma$ subunits of human $\mathrm{ENaCs}$ (Figure 8). In line with expectations, amiloride 4 produced a strong effect ( $68 \%$ inhibition of $\mathrm{ENaC}$ at $10 \mu \mathrm{M}$ ), whereas HMA 5 showed greatly reduced activity and 6-substituted analogs 11, 18, 25, and 26 showed similar or lower inhibition than HMA.

Amiloride 4, HMA 5, and analogs 18 and 26 were next tested in a rat model of diuresis to confirm that loss of ENaC inhibitory potency corresponded to an absence of diuretic and antikaliuretic effects in vivo (Figure 9). With the exception of vehicle controls, all animals were dosed with $25 \mathrm{mg} / \mathrm{kg}$ acetazolamide to induce diuresis ${ }^{64}$ prior to receiving $1.5 \mathrm{mg} / \mathrm{kg}$ of amiloride 4, HMA 5, 18, or 26. Urine was collected over a $6 \mathrm{~h}$ period following dosing. Consistent with previous reports, amiloride 4 showed potent diuretic and antikaliuretic effects, increasing urine volume and sodium excretion by $34 \%$ and $159 \%$, respectively, while decreasing potassium excretion by $92 \%$ relative to acetazolamide-treated controls $\left(\mathrm{Na}^{+} / \mathrm{K}^{+}\right.$ excretion ratio $=63, p<0.0001$ for all measurements) ${ }^{64}$ Compound 18 did not significantly decrease $\mathrm{K}^{+}$excretion, while HMA 5 and 2-methoxypyrimidine analog 26 showed a very slight potassium-sparing effect ( $24 \%$ reduction, $p<0.001$ ). HMA 5, 18, and 26 did not alter $\mathrm{Na}^{+}$excretion or urine volume, and the $\mathrm{Na}^{+} / \mathrm{K}^{+}$excretion ratios for the three compounds were no different to acetazolamide treated controls (range $=2.44-2.68$ ).

\section{Experimental Lung Metastasis Model.}

Two analogs were advanced to anticancer pilot studies to establish efficacy in vivo. Compounds 18 and 26 were selected as representative examples showing different potency and polarity $\left(18 \log D_{7.4}=5.1,26 \log D_{7.4}=3.4\right)$. In the first study, amiloride 4 and 6substituted HMA analogs 18 and 26 were examined for antimetastasis effects in an experimental mouse model of late-stage lung metastasis. Luciferase-tagged HT-1080 human fibrosarcoma cells were used in the model after confirming cell surface expression of uPA/ uPAR (Supporting Information Figure S1). Assays of lung homogenates following organ 
harvest at end point were used to quantitate lung tumor burden and response to treatment. Animals received $2.5 \times 10^{5}$ HT-1080 cells via lateral tail vein injection on day 0 and were weighed and scored for clinical signs (scoresheet Supporting Information Figure S2) over 21 days. Animals received $7.5 \mathrm{mg} \mathrm{kg}^{-1} \mathrm{day}^{-1}$ of compound (or vehicle) via ip injection from day -1 to day 20 . This dose was selected based on extensive in vivo pharmacokinetic profiling and represented a compromise between tolerability and attempting to demonstrate efficacy with compounds that show reduced potency against murine uPA. ${ }^{65}$

Amiloride 4 did not significantly inhibit metastasis in this model at the concentration tested. Benzofuranyl analog 18 decreased metastasis by $21 \%(p=0.0297)$ relative to control, but there was no statistically significant difference between amiloride and 18 (Figure 10). 2Methoxypyrimidine analog $\mathbf{2 6}$ significantly decreased lung metastasis $(33 \%, p=0.0007)$.

\section{Orthotopic Model of Pancreatic Cancer.}

The in vivo antitumor and antimetastasis effects of $\mathbf{1 8}$ and $\mathbf{2 6}$ were evaluated alongside the standard of care drug gemcitabine in an orthotopic xenograft mouse model of pancreatic cancer (Figure 11). Briefly, female athymic nude mice (BALBc nu/nu, 8-11 weeks of age) were anesthetized, an incision was made in the left flank, and the spleen and tail of the pancreas were externalized. A mixture of human pancreatic cancer cells (AsPC-1) $1 \times 10^{6}$ and human pancreatic stellate cells (hPSCs) $1 \times 10^{6}$ in $50 \mu \mathrm{L}$ of PBS was injected into the tail of the pancreas. uPA expression was confirmed in both cell lines by Western blot analysis (Supporting Information Figure S3). ${ }^{66}$ The pancreas and spleen were then reinternalized, and the wound was closed. Seven days after cell implantation, mice were randomized to receive treatment with gemcitabine $(75 \mathrm{mg} / \mathrm{kg}$ po twice weekly), 18, or $\mathbf{2 6}$ $(10 \mathrm{mg} / \mathrm{kg}$ ip daily) for 28 days ( $n=4$ mice/group). The higher dose (relative to the lung metastasis model) was chosen to increase the likelihood of observing efficacy. In line with previous observations in this model, gemcitabine showed only modest inhibitory effects on the primary tumor. ${ }^{67}$ Measurement of tumor volume at end point revealed that 18 and 26 produced similar reductions to gemcitabine on the primary mass (Figure 11A). Notably, liver metastases (key site in this model) were reduced in mice treated with compounds $\mathbf{1 8}$ and $\mathbf{2 6}$ compared to those treated with gemcitabine, with no macroscopic metastatic lesions observed in any mice that received 26 (Figure 11B). The absence of liver metastases in all animals treated with $\mathbf{2 6}$ was confirmed by histology (Supporting Information Figure S4).

\section{CONCLUSIONS}

This study reveals that 6-substituted HMA derivatives are potent inhibitors of human uPA with potential for development into a new class of anticancer drugs. Access to a diverse series of 6-(hetero)aryl analogs was afforded via a simple two step Suzuki-Miyaura/acyl guanidinylation approach using commercial boronic acids/esters and a common 6chloropyrazine intermediate 7 . The key intermediate was easily synthesized on multigram scale in a single step from commercial reagents. Buchwald-Hartwig chemistry was established on a related 6-bromopyrazine scaffold to access 6-aminopyrimidine HMA derivative 41 . Given the general reactivity of halopyrazines in transition-metal-catalyzed cross-coupling reactions, ${ }^{68}$ it is likely that a great many other substituents could be 
appended at the 6-position of HMA using this chemistry. Screening of such a library against proteins that reportedly bind to HMA (e.g., $\mathrm{P} 2 \mathrm{X}_{7},{ }^{69} \mathrm{NHE} 1,{ }^{70} \mathrm{HIV}-1 \mathrm{Vpu},{ }^{71}$ influenza A M2, ${ }^{72}$ GnRHR, ${ }^{73}$ GABA-A $\rho-1,{ }^{74}$ ASIC1a, ${ }^{75}$ and GIRK $)^{76}$ could help to identify more potent and selective probes for these targets, and possibly therapeutic leads.

Leading compounds from the study showed uPA inhibitory potencies more than 10-fold higher than HMA, with X-ray crystallography revealing that the gains arose from interactions formed between the appended 6-substituents and uPA's S1 $\beta$ pocket. High target selectivity was demonstrated across the serine hydrolase superfamily, and selected analogs showed no activity against ENaCs in vitro or $\mathrm{K}^{+}$-sparing/diuretic effects in vivo. Analogs carrying 2-benzofuranyl and 5-pyrimidinyl substituents emerged as distinct lead classes with differing physicochemical properties and human cell cytotoxicity profiles. Examples from each class were found to inhibit formation of lung metastases in a mouse experimental metastasis model, and 2-methoxypyrimidine analog 26 completely inhibited formation of liver macrometastases in a mouse pancreatic cancer model. In summary, this work reports the successful repurposing of the diuretic amiloride into potent uPA inhibitors with attractive pharmacological properties for further evaluation as a novel class as anticancer agents for treating uPA-driven malignancies.

\section{EXPERIMENTAL SECTION}

\section{Chemistry: General.}

All solvents and chemicals were purchased and used without further purification except ${ }^{i} \mathrm{PrOH}$, which was distilled from $\mathrm{BaO}$ and stored over $4 \AA$ molecular sieves under Ar. Progress of reactions was monitored using Merck silica gel $60 \mathrm{~F}_{254}$ TLC plates with EtOAc/ pet. spirit mixtures as eluent. Compound spots on TLC plates were visualized under UV light at $254 \mathrm{~nm}$. Column chromatography was performed using Merck silica gel 60 (230-400 mesh). Melting points (mp) were measured in open capillaries using a Griffin analog melting point apparatus (Thermo-Fisher Scientific, Waltham, MA, USA) and are uncorrected. ${ }^{1} \mathrm{H}$ and ${ }^{13} \mathrm{C}$ NMR spectra were recorded using a Varian Inova 500 or Varian Premium Shielded 500 (499.74 MHz for ${ }^{1} \mathrm{H} ; 125.66 \mathrm{MHz}$ for ${ }^{13} \mathrm{C}$ ) in deuterated solvents. Chemical shifts are reported in parts per million ( $\delta \mathrm{ppm})$ relative to TMS for ${ }^{1} \mathrm{H}$ or the solvent peak for ${ }^{13} \mathrm{C}$. High-resolution mass spectrometry (HRMS) analysis was performed using a Waters XEVO QToF mass spectrometer (Waters Corporation, Milford, MA, USA) with leucine encephalin as internal standard. Purity of all final compounds was confirmed at $>95 \%$ using high performance liquid chromatography (HPLC). HPLC analyses were performed using a Shimadzu CLASS-VP LC10 analytical HPLC system (Shimadzu Corporation, Kyoto, Japan) with detection at $254 \mathrm{~nm}$. Gradient elutions were performed using mixtures of solvent $100 \% \mathrm{H}_{2} \mathrm{O} / 0.1 \%$ TFA and $100 \% \mathrm{CH}_{3} \mathrm{CN} / 0.1 \%$ TFA on a Waters VisionHT $3 \mu \mathrm{m} \mathrm{C18} \mathrm{column}(150 \mathrm{~mm} \times 4.6 \mathrm{~mm})$ at a flow rate of $0.5 \mathrm{~mL} \cdot \mathrm{min}^{-1}$. Column temperature was not controlled. Only one previous 6-substituted HMA analog (6-phenyl HMA) has been reported, but no characterization or uPA inhibition data were described. ${ }^{77}$ 


\section{Chemistry: Experimental Procedures and Compound Characterization. Synthesis of Methyl 3-Amino-6-chloro-5-hexamethyleneimine-2-pyrazinecarboxylate (7).}

To a suspension of methyl 3-amino-5,6-dichloro-2-pyrazinecarboxylate $(5.55 \mathrm{~g}, 25.0 \mathrm{mmol})$ in 2-propanol $(50 \mathrm{~mL})$ was added hexamethyleneimine $(2.73 \mathrm{~g}, 3.10 \mathrm{~mL}, 27.5 \mathrm{mmol})$. DIPEA ( $3.55 \mathrm{~g}, 4.79 \mathrm{~mL}, 27.5 \mathrm{mmol})$ was added, and the reaction mixture was heated at reflux for $2 \mathrm{~h}$. The reaction mixture was allowed to cool to room temperature, which caused the product to crystallize. The solid product was collected by vacuum filtration and washed with cold 2-propanol $(2 \times 10 \mathrm{~mL})$ and then diethyl ether $(10 \mathrm{~mL})$. After drying, the pure product was obtained as pink crystals $(6.1 \mathrm{~g}, 86 \%)$. Mp 109-111 ${ }^{\circ} \mathrm{C} .{ }^{1} \mathrm{H} \mathrm{NMR}\left(\mathrm{CDCl}_{3}\right): \delta$ 6.13 (br s, 2H), $3.89(\mathrm{~s}, 3 \mathrm{H}), 3.82(\mathrm{t}, J=6.0 \mathrm{~Hz}, 4 \mathrm{H}), 1.84(\mathrm{~s}, 4 \mathrm{H}), 1.58(\mathrm{~s}, 4 \mathrm{H}) .{ }^{13} \mathrm{C} \mathrm{NMR}$ $\left(\mathrm{CDCl}_{3}\right): \delta 166.4,153.7,153.1,120.3,111.3,52.0,51.1,28.4,26.7$. MS (ESI): $\mathrm{m} / z 285$ (M $+\mathrm{H})^{+}, 307(\mathrm{M}+\mathrm{Na})^{+}$. HRMS $\left(\mathrm{ESI}^{+}\right)$Anal. For $\mathrm{C}_{12} \mathrm{H}_{17} \mathrm{~N}_{4} \mathrm{NaO}_{2}{ }^{+}$: calcd mass 307.0932, found 307.0938 .

\section{General Method A: Suzuki-Miyaura Coupling Reactions.}

Methyl 3-amino-6-chloro-5-hexamethyleneimine-2-pyrazine-carboxylate 7 (1 equiv) was combined with $\mathrm{K}_{2} \mathrm{CO}_{3}$ (10 equiv), the appropriate boronic acid (1.5 equiv), and $\mathrm{Pd}\left(\mathrm{PPh}_{3}\right)_{4}$ $(5 \mathrm{~mol} \%)$ in a two-neck round-bottom flask. The flask was connected to a condenser and purged with nitrogen. A 4:1 dry toluene/ $\mathrm{MeOH}$ mixture $(60 \mathrm{~mL})$ was added via syringe, and the reaction mixture was heated at reflux for $0.5-18 \mathrm{~h}$. The mixture was allowed to cool to room temperature and filtered through Celite $(10 \times 3 \mathrm{~cm}$, eluting with $3 \times 20 \mathrm{~mL}$ of EtOAc). The filtrate was evaporated to dryness and the residue purified by silica gel flash column chromatography using EtOAc/pet. spirit.

\section{Methyl 3-Amino-5-(azepan-1-yl)-6-(4-(methylthio)-phenyl)-pyrazine-2-carboxylate (10a).}

General method A using 4-(methylthio)phenylboronic acid ( $93 \mathrm{mg}, 0.545 \mathrm{mmol})$ afforded 10a as a yellow solid; yield 77\% (104 mg, $0.279 \mathrm{mmol})$. Mp 132-134 ${ }^{\circ} \mathrm{C} .{ }^{1} \mathrm{H}$ NMR (500 $\left.\mathrm{MHz}, \mathrm{CDCl}_{3}\right) \delta 7.42(\mathrm{~d}, J=8.1 \mathrm{~Hz}, 2 \mathrm{H}), 7.25(\mathrm{~d}, J=8.1 \mathrm{~Hz}, 2 \mathrm{H}), 6.20(\mathrm{bs}, 2 \mathrm{H}), 3.88$ (s, $3 \mathrm{H}), 3.36(\mathrm{t}, J=5.5 \mathrm{~Hz}, 4 \mathrm{H}),, 2.48(\mathrm{~s}, 3 \mathrm{H}), 1.62(\mathrm{~s}, 4 \mathrm{H}), 1.43(\mathrm{~s}, 4 \mathrm{H}) .{ }^{13} \mathrm{C}$ NMR $(126 \mathrm{MHz}$, CDCl3) $\delta 167.4,154.8,153.4,137.8,137.5,131.2,128.3,126.8,112.5,51.9,51.0,28.0$, 27.1, 16.1. HRESI-MS $m / z\left(\mathrm{M}+\mathrm{H}^{+}\right)$Anal. for $\mathrm{C}_{19} \mathrm{H}_{25} \mathrm{~N}_{4} \mathrm{O}_{2} \mathrm{~S}$ : calcd mass, 373.1698. Found: 373.1688 . Anal. HPLC $\left(70: 30 \mathrm{H}_{2} \mathrm{O} / 0.1 \%\right.$ TFA/CH 3 CN/0.1\% TFA $\rightarrow 100 \%$ $\mathrm{CH}_{3} \mathrm{CN} / 0.1 \%$ TFA over $30 \mathrm{~min}, t_{\mathrm{R}}=36.1 \mathrm{~min}$ ).

\section{Methyl 3-Amino-5-(azepan-1-yl)-6-(4-(trifluoromethyl)-phenyl)-pyrazine-2-carboxylate (11a).}

General method A using 4-(trifluoromethyl)phenylboronic acid (570 mg, $3.0 \mathrm{mmol}$ ) afforded 11a as a white solid; yield $64 \%$ (505 mg, $1.28 \mathrm{mmol}) . \mathrm{Mp} 158-160{ }^{\circ} \mathrm{C} .{ }^{1} \mathrm{H}$ NMR (500 MHz, $\mathrm{CDCl}_{3}$ ): $\delta 7.63$ (s, 4H), 6.13 (br s, 2H, $\mathrm{NH}_{2}$ ), 3.89 (s, 3H), $3.34(\mathrm{~s}, 4 \mathrm{H}), 1.65$ (s, $4 \mathrm{H}), 1.45$ (s, 4H). ${ }^{13} \mathrm{C}$ NMR $\left(126 \mathrm{MHz}, \mathrm{CDCl}_{3}\right): \delta 167.4,154.9,153.7,144.6,129.9,129.3$, 128.2, 125.6, 125.1, 113.2, 52.1, 51.3, 28.1, 27.3. HRESI-MS $m / z\left(\mathrm{M}+\mathrm{Na}^{+}\right)$Anal. For $\mathrm{C}_{19} \mathrm{H}_{21} \mathrm{~F}_{3} \mathrm{~N}_{4} \mathrm{NaO}_{2}$ : calcd mass 417.1526. Found 417.1514. Anal.-HPLC $\left(100 \% \mathrm{H}_{2} \mathrm{O} / 0.1 \%\right.$ $\mathrm{TFA} \rightarrow 100 \% \mathrm{CH}_{3} \mathrm{CN} / 0.1 \%$ TFA over $30 \mathrm{~min}, t_{\mathrm{R}}=29.3 \mathrm{~min}$ ). 


\section{Methyl 3-Amino-5-(azepan-1-yl)-6-(benzo[d][1,3]dioxol-5-yl)pyrazine-2-carboxylate (12a).}

General method A using benzo-[d][1,3]dioxol-5-ylboronic acid (85 mg, $0.515 \mathrm{mmol})$ afforded $12 \mathrm{a}$ as a yellow solid; yield $51 \%$ (66 mg, $0.178 \mathrm{mmol}) . \mathrm{Mp} 156-158{ }^{\circ} \mathrm{C} .{ }^{1} \mathrm{H}$ NMR $\left(500 \mathrm{MHz}, \mathrm{CDCl}_{3}\right) \delta 7.02(\mathrm{~s}, 1 \mathrm{H}), 6.93(\mathrm{~d}, J=8.0 \mathrm{~Hz}, 1 \mathrm{H}), 6.80(\mathrm{~d}, J=8.0 \mathrm{~Hz}, 1 \mathrm{H}), 5.96(\mathrm{~s}$, $2 \mathrm{H}), 3.88(\mathrm{~s}, 4 \mathrm{H}), 3.38(\mathrm{~s}, 4 \mathrm{H}), 1.63(\mathrm{~s}, 4 \mathrm{H}), 1.44(\mathrm{~s}, 4 \mathrm{H}) .{ }^{13} \mathrm{C} \mathrm{NMR}\left(126 \mathrm{MHz}, \mathrm{CDCl}_{3}\right) \delta$ 167.6, 154.9, 153.5, 147.9, 147.1, 134.9, 131.5, 121.7, 112.2, 108.7, 108.3, 101.1, 51.9, 51.0, 28.1, 27.1. HRESI-MS $m / z\left(\mathrm{M}+\mathrm{H}^{+}\right)$Anal. for $\mathrm{C}_{19} \mathrm{H}_{23} \mathrm{~N}_{4} \mathrm{O}_{4}$ : calcd mass 371.1719. Found 371.1734. Anal. HPLC $\left(100 \mathrm{H}_{2} \mathrm{O} / 0.1 \%\right.$ TFA $\rightarrow 100 \% \mathrm{CH}_{3} \mathrm{CN} / 0.1 \%$ TFA over 30 $\left.\min , t_{\mathrm{R}}=31.8 \mathrm{~min}\right)$.

\section{Methyl 3-Amino-5-(azepan-1-yl)-6-(isoquinolin-4-yl)-pyrazine-2-carboxylate (13a).}

General method A using isoquinolin-4-ylboronic acid (271 mg, $1.06 \mathrm{mmol})$ afforded 13a as a brown solid; yield 58\% (156 mg, $0.413 \mathrm{mmol}) . \mathrm{Mp} 140-142{ }^{\circ} \mathrm{C} .{ }^{1} \mathrm{H} \mathrm{NMR}(500 \mathrm{MHz}$, $\left.\mathrm{CDCl}_{3}\right) \delta 9.23(\mathrm{~s}, 1 \mathrm{H}), 8.58(\mathrm{~s}, 1 \mathrm{H}), 8.00(\mathrm{~d}, J=8.0 \mathrm{~Hz}, 1 \mathrm{H}), 7.81(\mathrm{~d}, J=8.0 \mathrm{~Hz}, 1 \mathrm{H}), 7.68$ (t, $J=7.5 \mathrm{~Hz}, 1 \mathrm{H}), 7.62(\mathrm{t}, J=7.5 \mathrm{~Hz}, 1 \mathrm{H}), 3.86(\mathrm{~s}, 3 \mathrm{H}), 3.21(\mathrm{t}, J=5.5 \mathrm{~Hz}, 4 \mathrm{H}), 1.47(\mathrm{~s}$, $4 \mathrm{H}), 1.38(\mathrm{~s}, 4 \mathrm{H}) .{ }^{13} \mathrm{C} \mathrm{NMR}\left(126 \mathrm{MHz}, \mathrm{CDCl}_{3}\right) \delta 167.3,155.8,154.0,152.1,143.0,134.3$, $131.8,130.8,128.4,128.0,127.4,126.7,124.5,112.9,52.0,50.4,27.8,26.8$. HRESI-MS $\mathrm{m} / \mathrm{Z}\left(\mathrm{M}+\mathrm{Na}^{+}\right) 400$, Anal. for $\mathrm{C}_{21} \mathrm{H}_{24} \mathrm{~N}_{5} \mathrm{NaO}_{2}$ : calcd mass 400.1749. Found 400.1747. Anal. $\operatorname{HPLC}\left(100 \% \mathrm{H}_{2} \mathrm{O} / 0.1 \% \mathrm{TFA} \rightarrow 100 \% \mathrm{CH}_{3} \mathrm{CN} / 0.1 \%\right.$ TFA over $\left.30 \mathrm{~min}, t_{\mathrm{R}}=22.3 \mathrm{~min}\right)$.

\section{Methyl 3-Amino-5-(azepan-1-yl)-6-(1-methyl-1 H-pyrazol-4-yl)pyrazine-2-carboxylate (14a).}

General method A using 1methyl-1-H-pyrazol-4-ylboronic acid $(110 \mathrm{mg}, 0.526 \mathrm{mmol})$ afforded $14 \mathrm{a}$ as a yellow solid; yield $77 \%(89 \mathrm{mg}, 0.269 \mathrm{mmol})$. Mp 134-136 ${ }^{\circ} \mathrm{C} .{ }^{1} \mathrm{H}$ NMR $\left(500 \mathrm{MHz}, \mathrm{CDCl}_{3}\right) \delta 7.64(\mathrm{~s}, 1 \mathrm{H}), 7.57(\mathrm{~s}, 1 \mathrm{H}), 6.17(\mathrm{bs}, 2 \mathrm{H}), 3.91(\mathrm{~s}, 3 \mathrm{H}), 3.89(\mathrm{~s}, 3 \mathrm{H})$, $3.47(\mathrm{t}, J=6 \mathrm{~Hz}, 4 \mathrm{H}), 1.66(\mathrm{~s}, 4 \mathrm{H}), 1.47(\mathrm{~s}, 4 \mathrm{H}) .{ }^{13} \mathrm{C} \mathrm{NMR}\left(126 \mathrm{MHz}, \mathrm{CDCl}_{3}\right) \delta 167.4$, 155.5, 153.4, 138.7, 129.1, 125.3, 122.6, 112.4, 52.1, 51.2, 39.0, 28.0, 27.2. HRESI-MS $\mathrm{m} / \mathrm{z}$ $\left(\mathrm{M}+\mathrm{H}^{+}\right)$331, Anal. for $\mathrm{C}_{16} \mathrm{H}_{23} \mathrm{~N}_{6} \mathrm{O}_{2}$ : calcd mass 331.1882. Found 331.1894. Anal. HPLC $\left(70: 30 \mathrm{H}_{2} \mathrm{O} / 0.1 \% \mathrm{TFA} / \mathrm{CH}_{3} \mathrm{CN} / 0.1 \% \mathrm{TFA} \rightarrow 100 \% \mathrm{CH}_{3} \mathrm{CN} / 0.1 \%\right.$ TFA over $30 \mathrm{~min}, t_{\mathrm{R}}=$ $25.2 \mathrm{~min})$.

\section{Methyl 3-Amino-5-(azepan-1-yl)-6-(1-(2-morpholinoethyl)-1H-pyrazol-4-yl)pyrazine-2- carboxylate (15a).}

General method A using 1-(2-morpholinoethyl)-1H-pyrazol-4-ylboronic acid (165 mg, $0.536 \mathrm{mmol}$ ) afforded $15 \mathrm{a}$ as a yellow solid; yield $80 \%$ (119 mg, $0.277 \mathrm{mmol}) . \mathrm{Mp}$ 132-134 ${ }^{\circ} \mathrm{C} .{ }^{1} \mathrm{H}$ NMR $\left(500 \mathrm{MHz}, \mathrm{CDCl}_{3}\right) \delta 7.70(\mathrm{~s}, 1 \mathrm{H}), 7.61$ (s, $\left.1 \mathrm{H}\right), 6.24$ (bs, 2H), 4.25 (s, 2H), 3.89 (s, 3H), $3.69(\mathrm{~s}, 4 \mathrm{H}), 3.46(\mathrm{~s}, 4 \mathrm{H}), 2.84(\mathrm{~s}, 2 \mathrm{H}), 2.50(\mathrm{~s}, 4 \mathrm{H}), 1.65(\mathrm{~s}, 4 \mathrm{H}), 1.47$ (s, 4H). ${ }^{13} \mathrm{C}$ NMR $\left(126 \mathrm{MHz}, \mathrm{CDCl}_{3}\right) \delta 167.2,155.5,153.3,138.6,128.7,125.2,122.1$ 112.4, 66.9, 58.2, 53.7, 51.9, 51.1, 49.6, 27.9 27.0. HRESI-MS $m / z\left(\mathrm{M}+\mathrm{H}^{+}\right)$, Anal. for $\mathrm{C}_{22} \mathrm{H}_{36} \mathrm{~N}_{7} \mathrm{O}_{3}$ : calcd mass 430.2567. Found 430.2553. Anal. HPLC $\left(70: 30 \mathrm{H}_{2} \mathrm{O} / 0.1 \%\right.$ $\mathrm{TFA} / \mathrm{CH}_{3} \mathrm{CN} / 0.1 \% \mathrm{TFA} \rightarrow 100 \% \mathrm{CH}_{3} \mathrm{CN} / 0.1 \%$ TFA over $\left.30 \mathrm{~min}, t_{\mathrm{R}}=21.6 \mathrm{~min}\right)$.

\section{Methyl 3-Amino-5-(azepan-1-yl)-6-(1H-indol-2-yl)-pyrazine-2-carboxylate (16a).}

General method A using 1H-indol-2-ylboronic acid $(278 \mathrm{mg}, 1.73 \mathrm{mmol})$ afforded $16 \mathrm{a}$ as an orange solid; yield $34 \%$ (87.5 mg, $0.239 \mathrm{mmol}) . \mathrm{Mp} 152-154{ }^{\circ} \mathrm{C} .{ }^{1} \mathrm{H}$ NMR $(500 \mathrm{MHz}$, 
$\left.\mathrm{CDCl}_{3}\right) \delta 9.14(\mathrm{~s}, 1 \mathrm{H}), 7.57(\mathrm{~d}, 1 \mathrm{H}), 7.37(\mathrm{~d}, 1 \mathrm{H}, J=7.7 \mathrm{~Hz}), 7.16(\mathrm{t}, 1 \mathrm{H}, J=7.0 \mathrm{~Hz}), 7.08$ $(\mathrm{t}, 1 \mathrm{H}, J=7.0 \mathrm{~Hz}), 6.52(\mathrm{~s}, 1 \mathrm{H}), 3.93(\mathrm{~s}, 3 \mathrm{H}), 3.58(\mathrm{t}, J=5.75 \mathrm{~Hz}, 4 \mathrm{H}), 1.70(\mathrm{~s}, 4 \mathrm{H}), 1.50(\mathrm{~s}$, $4 \mathrm{H}) .{ }^{13} \mathrm{C}$ NMR $\left(126 \mathrm{MHz}, \mathrm{CDCl}_{3}\right) \delta 167.2,155.1,153.2,136.0,135.7,129.0,124.5,122.4$, 120.7, 119.90, 112.70, 111.0, 102.0, 52.1, 51.7, 28.1, 27.5. HRESI-MS $m / z\left(\mathrm{M}+\mathrm{H}^{+}\right) 388$, Anal. for $\mathrm{C}_{20} \mathrm{H}_{24} \mathrm{~N}_{5} \mathrm{O}_{2}$ : calcd mass 388.1730. Found 388.1731. Anal. HPLC $\left(100 \mathrm{H}_{2} \mathrm{O} / 0.1 \%\right.$ $\mathrm{TFA} \rightarrow 100 \% \mathrm{CH}_{3} \mathrm{CN} / 0.1 \%$ TFA over $30 \mathrm{~min}, t_{\mathrm{R}}=29.8 \mathrm{~min}$ ).

\section{Methyl 3-Amino-5-(azepan-1-yl)-6-(benzo[b]thiophen-2-yl)-pyrazine-2-carboxylate (17a).}

General method A using benzo[b]-thiophen-2-ylboronic acid (534 mg, $3.0 \mathrm{mmol}$ ) afforded $17 \mathrm{a}$ as a yellow solid; yield $78 \%(600 \mathrm{mg})$. Mp $146-148{ }^{\circ} \mathrm{C} .{ }^{1} \mathrm{H} \mathrm{NMR}\left(500 \mathrm{MHz}, \mathrm{CDCl}_{3}\right): \delta$ $1.48(\mathrm{~s}, 4 \mathrm{H}), 1.69$ (s, 4H), 3.51 (s, 4H), $3.91(\mathrm{~s}, 3 \mathrm{H}), 6.13(\mathrm{br} \mathrm{s}, 2 \mathrm{H}, \mathrm{NH} 2), 7.22(\mathrm{~s}, 1 \mathrm{H}), 7.21$ $(\mathrm{m}, 2 \mathrm{H}), 7.71(\mathrm{~d}, J=6.9 \mathrm{~Hz}, 1 \mathrm{H}), 7.80(\mathrm{~d}, \mathrm{~J}=7.0 \mathrm{~Hz}, 1 \mathrm{H}) .{ }^{13} \mathrm{C} \mathrm{NMR}\left(126 \mathrm{MHz}, \mathrm{CDCl}_{3}\right): \delta$ 167.3, 155.3, 153.6, 143.3, 140.2, 140.1, 125.4, 124.5, 124.4, 123.6, 122.3, 122.0, 113.2, 52.3, 51.3, 28.2, 27.4. HRESI-MS $m / z\left(\mathrm{M}+\mathrm{K}^{+}\right)$Anal. For $\mathrm{C}_{20} \mathrm{H}_{22} \mathrm{KN}_{4} \mathrm{O}_{3}$ calcd mass 405.1394. Found 405.1361. Anal.-HPLC $\left(100 \mathrm{H}_{2} \mathrm{O} / 0.1 \%\right.$ TFA $\rightarrow 100 \% \mathrm{CH}_{3} \mathrm{CN} / 0.1 \%$ TFA over $30 \mathrm{~min}, t_{\mathrm{R}}=31.9 \mathrm{~min}$ ).

\section{Methyl 3-Amino-5-(azepan-1-yl)-6-(benzofuran-2-yl)-pyrazine-2-carboxylate (18a).}

General method A using benzofuran-2-ylboronic acid $(486 \mathrm{mg}, 3.0 \mathrm{mmol})$ afforded 18a as a yellow solid; yield 57\% (420 mg). Mp 121-123 ${ }^{\circ} \mathrm{C} .{ }^{1} \mathrm{H}$ NMR (500 MHz, $\left.\mathrm{CDCl}_{3}\right): \delta 1.46$ (s, $4 \mathrm{H}), 1.66(\mathrm{~s}, 4 \mathrm{H}), 3.45(\mathrm{t}, J=5.8 \mathrm{~Hz}, 4 \mathrm{H}), 3.90(\mathrm{~s}, 3 \mathrm{H}), 6.13(\mathrm{br} \mathrm{s}, 2 \mathrm{H}, \mathrm{NH} 2), 6.93(\mathrm{~s}, 1 \mathrm{H})$, $7.21(\mathrm{t}, J=7.3 \mathrm{~Hz}, 1 \mathrm{H}), 7.25(\mathrm{t}, J=7.2 \mathrm{~Hz}, 1 \mathrm{H}), 7.47(\mathrm{~d}, \mathrm{~J}=7.9 \mathrm{~Hz}, 1 \mathrm{H}), 7.56(\mathrm{~d}, J=7.5$ $\mathrm{Hz}, 1 \mathrm{H}) .{ }^{13} \mathrm{C}$ NMR $\left(126 \mathrm{MHz}, \mathrm{CDCl}_{3}\right): \delta 167.2,164.9,154.8,154.6,129.2,124.4,123.1$, 121.4, 121.2, 112.9, 111.5, 105.1, 52.2, 50.2, 28.2, 27.3. HRESI-MS $\mathrm{m} / \mathrm{z}\left(\mathrm{M}+\mathrm{H}^{+}\right)$Anal. for $\mathrm{C}_{20} \mathrm{H}_{23} \mathrm{~N}_{4} \mathrm{O}_{2}$ : calcd mass 367.1831. Found. 367.1770. Anal.-HPLC (70:30 $\mathrm{H}_{2} \mathrm{O} / 0.1 \%$ $\mathrm{CH}_{3} \mathrm{CN} / 0.1 \% \mathrm{TFA} \rightarrow 100 \% \mathrm{CH}_{3} \mathrm{CN} / 0.1 \%$ TFA over $\left.30 \mathrm{~min}, t_{\mathrm{R}}=29.5 \mathrm{~min}\right)$.

\section{Methyl 3-Amino-5-(azepan-1-yl)-6-(4-fluorobenzofuran-2yl)-pyrazine-2-carboxylate (19a).}

General method A using 4-fluorobenzofuran-2-ylboronic acid (95 mg, $0.531 \mathrm{mmol})$ afforded 19a as a yellow solid; yield $58 \%$ (80 mg, $0.210 \mathrm{mmol})$. Mp 108-110 ${ }^{\circ} \mathrm{C} .{ }^{1} \mathrm{H}$ NMR (126 $\left.\mathrm{MHz}, \mathrm{CDCl}_{3}\right): 7.27(\mathrm{~d}, 1 \mathrm{H}), 7.20(\mathrm{~m}, 1 \mathrm{H}), 6.99(\mathrm{~s}, 1 \mathrm{H}), 6.93(\mathrm{t}, 1 \mathrm{H}, J=8.5 \mathrm{~Hz}), 3.91(\mathrm{~s}$, $3 \mathrm{H}), 3.46(\mathrm{~s}, 4 \mathrm{H}), 1.69(\mathrm{~s}, 4 \mathrm{H}), 1.49(\mathrm{~s}, 4 \mathrm{H}) .{ }^{13} \mathrm{C} \mathrm{NMR}\left(126 \mathrm{MHz}, \mathrm{CDCl}_{3}\right) \delta 167.0,156.9$, 156.2, 154.7, 154.0, 138.3, 124.6, 120.5, 118.0, 115.9, 110.4, 108.6, 107.6, 52.2, 50.0, 28.0, 27.1. HRESI-MS $m / z\left(\mathrm{M}+\mathrm{Na}^{+}\right)$Anal. for $\mathrm{C}_{20} \mathrm{H}_{21} \mathrm{FN}_{6} \mathrm{NaO}_{4}$ : calcd mass 407.1495. Found 407.1504. Anal. HPLC (70:30 $\mathrm{H}_{2} \mathrm{O} / 0.1 \% \mathrm{TFA} / \mathrm{CH}_{3} \mathrm{CN} / 0.1 \% \mathrm{TFA} \rightarrow 100 \% \mathrm{CH}_{3} \mathrm{CN} / 0.1 \%$ TFA over $30 \mathrm{~min}, t_{\mathrm{R}}=21.2 \mathrm{~min}$ ).

\section{Methyl-3-amino-5-(azepan-1-yl)-N-carbamimidoyl-6-(thiophen-2-yl)-pyrazine-2-carboxamide (20a).}

General method A using thiophen-2-ylboronic acid (70 mg, $0.549 \mathrm{mmol})$ afforded 20a as a light yellow solid; yield 85\% (99 mg, $0.299 \mathrm{mmol})$. Mp 118-120 ${ }^{\circ} \mathrm{C} .{ }^{1} \mathrm{H} \mathrm{NMR}(500 \mathrm{MHz}$, $\left.\mathrm{CDCl}_{3}\right) \delta 7.27(\mathrm{~d}, \mathrm{~J}=5 \mathrm{~Hz}, 1 \mathrm{H}), 7.03(\mathrm{~d}, J=3 \mathrm{~Hz}, 1 \mathrm{H}), 6.98(\mathrm{~m}, 1 \mathrm{H}), 3.89(\mathrm{~s}, 3 \mathrm{H}), 3.43(\mathrm{t}, J$ $=5.7 \mathrm{~Hz}, 4 \mathrm{H}), 1.67(\mathrm{~s}, 4 \mathrm{H}), 1.47(\mathrm{~s}, 4 \mathrm{H}) .{ }^{13} \mathrm{C} \mathrm{NMR}\left(126 \mathrm{MHz}, \mathrm{CDCl}_{3}\right) \delta 167.3,155.2$, $153.5,142.8,126.9,126.0,125.8,125.5,112.7,52.1,51.1,28.2,27.3$. HRESI-MS $\mathrm{m} / \mathrm{z}(\mathrm{M}+$ 
$\mathrm{Na}^{+}$) Anal. for $\mathrm{C}_{16} \mathrm{H}_{20} \mathrm{~N}_{4} \mathrm{NaO}_{2}$ : calcd mass 355.1205. Found 355.1193. Anal. HPLC (100 $\mathrm{H}_{2} \mathrm{O} / 0.1 \% \mathrm{TFA} \rightarrow 100 \% \mathrm{CH}_{3} \mathrm{CN} / 0.1 \%$ TFA over $\left.30 \mathrm{~min}, t_{\mathrm{R}}=28.8 \mathrm{~min}\right)$.

Methyl 3-Amino-5-(azepan-1-yl)-6-(thiophen-3-yl)-pyrazine-2-carboxylate (21a).

General method A using thiophen-3-ylboronic acid (75 mg, $0.584 \mathrm{mmol})$ afforded 21a as a white solid; yield $85 \%$ (100 mg, $0.302 \mathrm{mmol}$ ). Mp 118-120 ${ }^{\circ} \mathrm{C} .{ }^{1} \mathrm{H}$ NMR $(500 \mathrm{MHz}$, $\left.\mathrm{CDCl}_{3}\right) \delta 7.38(\mathrm{~s}, 1 \mathrm{H}), 7.29(\mathrm{~m}, 1 \mathrm{H}), 7.25(\mathrm{~d}, J=5 \mathrm{~Hz}, 1 \mathrm{H}), 3.88(\mathrm{~s}, 3 \mathrm{H}), 3.38(\mathrm{t}, J=5.8 \mathrm{~Hz}$, $4 \mathrm{H}), 1.63(\mathrm{~s}, 4 \mathrm{H}), 1.44(\mathrm{~s}, 4 \mathrm{H}) .{ }^{13} \mathrm{C} \mathrm{NMR}\left(126 \mathrm{MHz}, \mathrm{CDCl}_{3}\right) \delta 167.4,155.3,153.5,141.3$, 128.1, 127.9, 125.4, 122.8, 112.2, 52.0, 50.9, 28.0, 27.1. HRESI-MS $m / z\left(\mathrm{M}+\mathrm{H}^{+}\right)$Anal. For $\mathrm{C}_{16} \mathrm{H}_{21} \mathrm{~N}_{4} \mathrm{NaO}_{2} \mathrm{~S}$ : calcd mass 355.1205 . Found 355.1197. Anal. HPLC $\left(100 \mathrm{H}_{2} \mathrm{O} / 0.1 \%\right.$ $\mathrm{TFA} \rightarrow 100 \% \mathrm{CH}_{3} \mathrm{CN} / 0.1 \%$ TFA over $30 \mathrm{~min}, t_{\mathrm{R}}=28.3 \mathrm{~min}$ ).

\section{Methyl 3-Amino-5-(azepan-1-yl)-6-(furan-2-yl)-pyrazine-2-carboxylate (22a).}

General method A using furan-2-ylboronic acid $(59 \mathrm{mg}, 0.526 \mathrm{mmol})$ afforded 22a as an off white solid; yield 98\% (111 mg, $0.352 \mathrm{mmol})$. Mp 116-118 ${ }^{\circ} \mathrm{C} .{ }^{1} \mathrm{H}$ NMR (500 MHz, $\left.\mathrm{CDCl}_{3}\right): \delta 7.41(\mathrm{~s}, 1 \mathrm{H}), 6.54(\mathrm{~d}, J=2.3 \mathrm{~Hz}, 1 \mathrm{H}), 6.45(\mathrm{~s}, 1 \mathrm{H}), 6.13\left(\mathrm{br} \mathrm{s}, 2 \mathrm{H}, \mathrm{NH}_{2}\right), 3.89$ (s, $3 \mathrm{H}), 3.37(\mathrm{t}, J=5.5 \mathrm{~Hz}, 4 \mathrm{H}), 1.66(\mathrm{~s}, 4 \mathrm{H}), 1.47(\mathrm{~s}, 4 \mathrm{H}) .{ }^{13} \mathrm{C} \mathrm{NMR}\left(126 \mathrm{MHz}, \mathrm{CDCl}_{3}\right): \delta$ $167.1,154.8,154.0,152.2,141.4,122.0,112.1,111.6,108.6,52.0,50.0,28.0,26.9$. HRESIMS $m / z\left(\mathrm{M}+\mathrm{Na}^{+}\right)$Anal. for $\mathrm{C}_{16} \mathrm{H}_{21} \mathrm{~N}_{4} \mathrm{NaO}_{3}$ : calcd mass 339.1418. Found 339.1433. Anal.$\operatorname{HPLC}\left(100 \mathrm{H}_{2} \mathrm{O} / 0.1 \% \mathrm{TFA} \rightarrow 100 \% \mathrm{CH}_{3} \mathrm{CN} / 0.1 \%\right.$ TFA over $\left.30 \mathrm{~min}, t_{\mathrm{R}}=27.1 \mathrm{~min}\right)$.

\section{Methyl 3-Amino-5-(azepan-1-yl)-6-(furan-3-yl)-pyrazine-2-carboxylate (23a).}

General method A using furan-3-ylboronic acid (107 $\mathrm{mg}, 0.389 \mathrm{mmol})$ afforded 23a as a yellow solid; yield 93\% (114 mg, $0.360 \mathrm{mmol})$. Mp 122-124 ${ }^{\circ} \mathrm{C} .{ }^{1} \mathrm{H}$ NMR $(500 \mathrm{MHz}$, $\left.\mathrm{CDCl}_{3}\right) \delta 7.64(\mathrm{~s}, 1 \mathrm{H}), 7.41(\mathrm{~s}, 1 \mathrm{H}), 6.61(\mathrm{~s}, 1 \mathrm{H}), 6.20(\mathrm{bs}, 2 \mathrm{H}), 3.89(\mathrm{~s}, 3 \mathrm{H}), 3.48(\mathrm{t}, J=5$ $\mathrm{Hz}, 4 \mathrm{H}), 1.66$ (s, 4H), 1.47 (s, 4H). ${ }^{13} \mathrm{C} \mathrm{NMR}\left(126 \mathrm{MHz}, \mathrm{CDCl}_{3}\right) \delta 167.3,155.6,153.6$, 142.6, 140.6, 125.8, 124.8, 112.6, 111.2, 52.0, 51.0, 28.0, 27.1. HRESI-MS $m / z\left(\mathrm{M}+\mathrm{H}^{+}\right)$ Anal. for $\mathrm{C}_{16} \mathrm{H}_{21} \mathrm{~N}_{4} \mathrm{O}_{3}$ : calcd mass 316.1614. Found 316.1604. Anal. HPLC (70:30 $\mathrm{H}_{2} \mathrm{O} /$ $0.1 \% \mathrm{TFA} / \mathrm{CH}_{3} \mathrm{CN} / 0.1 \% \mathrm{TFA} \rightarrow 100 \% \mathrm{CH}_{3} \mathrm{CN} / 0.1 \%$ TFA over $\left.30 \mathrm{~min}, t_{\mathrm{R}}=30.9 \mathrm{~min}\right)$.

\section{Methyl 3-Amino-5-(azepan-1-yl)-6-(2-fluoropyridin-3-yl)-pyrazine-2-carboxylate (24a).}

General method A using 2-fluoropyridin-3-ylboronic acid (75 mg, $0.529 \mathrm{mmol}$ ) afforded 24a as a brown solid; yield 76\% (92 mg, $0.266 \mathrm{mmol})$. Mp 156-158 ${ }^{\circ} \mathrm{C} .{ }^{1} \mathrm{H}$ NMR (500 $\left.\mathrm{MHz}, \mathrm{CDCl}_{3}\right) \delta 8.17(\mathrm{~d}, J=4.3 \mathrm{~Hz}, 1 \mathrm{H}), 8.06(\mathrm{~m}, 1 \mathrm{H}), 7.29(\mathrm{t}, J=5.5 \mathrm{~Hz}, 1 \mathrm{H}), 3.89(\mathrm{~s}, 3 \mathrm{H})$, $3.36(\mathrm{~s}, J=5.7 \mathrm{~Hz}, 4 \mathrm{H}), 1.63(\mathrm{~s}, 4 \mathrm{H}), 1.46(\mathrm{~s}, 4 \mathrm{H}) .{ }^{13} \mathrm{C} \mathrm{NMR}\left(126 \mathrm{MHz}, \mathrm{CDCl}_{3}\right) \delta 167.2$, 161.3, 155.2, 154.1, 146.8, 141.1, 124.5, 123.8, 122.2, 113.3, 52.3, 50.3, 28.1, 27.3. HRESIMS $m / z\left(\mathrm{M}+\mathrm{Na}^{+}\right)$Anal. for $\mathrm{C}_{17} \mathrm{H}_{20} \mathrm{FN}_{5} \mathrm{NaO}_{2}$ : calcd mass 368.1499. Found 368.1489. Anal. HPLC $\left(100 \mathrm{H}_{2} \mathrm{O} / 0.1 \%\right.$ TFA $\rightarrow 100 \% \mathrm{CH}_{3} \mathrm{CN} / 0.1 \%$ TFA over $\left.30 \mathrm{~min}, t_{\mathrm{R}}=26.3 \mathrm{~min}\right)$.

\section{Methyl 3-Amino-5-(azepan-1-yl)-6-(pyrimidin-5-yl)-pyrazine-2-carboxylate (25a).}

General method A using pyrimidin-5-ylboronic acid (279 mg, $2.25 \mathrm{mmol})$ afforded 25a as a yellow solid; yield $91 \%$ (450 mg). Mp 176-178 ${ }^{\circ} \mathrm{C} .{ }^{1} \mathrm{H}$ NMR (500 MHz, $\mathrm{CDCl}_{3}$ ): $\delta 9.13$ (s, 1H), 8.89 (s, 2H), 6.13 (br s, 2H, NH2), 3.91 (s, 3H), 3.52 (t, $J=6.0 \mathrm{~Hz}, 4 \mathrm{H}), 1.68$ (s, 4H), 1.48 (s, 4H). ${ }^{13} \mathrm{C}$ NMR $\left(126 \mathrm{MHz}, \mathrm{CDCl}_{3}\right): \delta 167.1,157.1,155.7,155.3,153.9,134.9$, 
124.5, 114.5, 52.3, 51.5, 28.0, 27.4. HRESI-MS $m / z\left(\mathrm{M}+\mathrm{Na}^{+}\right)$Anal. for $\mathrm{C}_{16} \mathrm{H}_{20} \mathrm{~N}_{6} \mathrm{NaO}_{2}$ : calcd mass 351.1545. Found. 351.1535. Anal.-HPLC (70:30 $\mathrm{H}_{2} \mathrm{O} / 0.1 \%$ TFA/CH $3 \mathrm{CN} / 0.1 \%$ $\mathrm{TFA} \rightarrow 100 \% \mathrm{CH}_{3} \mathrm{CN} / 0.1 \%$ TFA over $\left.30 \mathrm{~min}, t_{\mathrm{R}}=23.4 \mathrm{~min}\right)$.

Methyl 3-Amino-5-(azepan-1-yl)-6-(2-methoxypyrimidin-5yl)-pyrazine-2-carboxylate (26a).

General method A using 2-methoxypyrimidin-5-ylboronic acid (79 mg, $0.513 \mathrm{mmol}$ ) afforded 26a as a yellow solid; yield $88 \%$ (111 mg, $0.310 \mathrm{mmol})$. Mp 168-170 ${ }^{\circ} \mathrm{C} .{ }^{1} \mathrm{H}$ NMR (500 MHz, $\mathrm{CDCl}_{3}$ ): 8.66 (s, 2H), 6.13 (br s, 2H, NH ), 4.05 (s, 3H), 3.90 (s, 3H), 3.38 (t, $J=$ $5.5 \mathrm{~Hz}, 4 \mathrm{H}), 1.68$ (s, 4H), $\delta 1.48$ (s, 4H). ${ }^{13} \mathrm{C}$ NMR (126 MHz, $\left.\mathrm{CDCl}_{3}\right): \delta 166.4,164.7$, $158.3,155.3,153.8,128.8,125.2,113.9,55.2,52.2,51.4,28.0,27.3$. HRESI-MS $m / z(\mathrm{M}+$ $\mathrm{H}^{+}$) Anal. for $\mathrm{C}_{17} \mathrm{H}_{22} \mathrm{~N}_{6} \mathrm{O}_{3}$ : calcd mass 359.1832. Found 359.1848. Anal.-HPLC (70:30 $\mathrm{H}_{2} \mathrm{O} / 0.1 \%$ TFA/ $\mathrm{CH}_{3} \mathrm{CN} / 0.1 \%$ TFA $\rightarrow 100 \% \mathrm{CH}_{3} \mathrm{CN} / 0.1 \%$ TFA over $30 \mathrm{~min}, t \mathrm{r}=28.0$ $\min )$.

\section{Methyl 3-Amino-5-(azepan-1-yl)-6-(2,4-dimethoxypyrimidin-5-yl)-pyrazine-2-carboxylate (27a).}

General method A using 2,4-dimethoxypyrimidin-5-ylboronic acid (79 $\mathrm{mg}, 0.350 \mathrm{mmol}$ ) afforded $27 \mathrm{a}$ as a yellow solid; yield $91 \%(124 \mathrm{mg}, 0.319 \mathrm{mmol})$. Mp $150-152{ }^{\circ} \mathrm{C} .{ }^{1} \mathrm{H}$ NMR $\left(500 \mathrm{MHz}, \mathrm{CDCl}_{3}\right) \delta 8.36(\mathrm{~s}, 1 \mathrm{H}), 4.03(\mathrm{~s}, 3 \mathrm{H}), 3.94(\mathrm{~s}, 3 \mathrm{H}), 3.87(\mathrm{~s}, 3 \mathrm{H}), 3.39(\mathrm{~s}, 4 \mathrm{H}), 1.60$ (s, 4H,), 1.46 (s, 4H). ${ }^{13} \mathrm{C}$ NMR (126 MHz, $\left.\mathrm{CDCl}_{3}\right) \delta 168.3,167.2,164.8,158.1,155.3$, 154.1, 123.8, 117.1, 112.6, 55.0, 54.1, 52.0, 50.0, 28.0, 27.0. HRESI-MS $\mathrm{m} / \mathrm{z}\left(\mathrm{M}+\mathrm{H}^{+}\right)$ Anal. for $\mathrm{C}_{18} \mathrm{H}_{25} \mathrm{~N}_{6} \mathrm{O}_{4}$ calcd mass 389.1937. Found389.1929. Anal.-HPLC $\left(70: 30 \mathrm{H}_{2} \mathrm{O} /\right.$ $0.1 \% \mathrm{TFA} / \mathrm{CH}_{3} \mathrm{CN} / 0.1 \% \mathrm{TFA} \rightarrow 100 \% \mathrm{CH}_{3} \mathrm{CN} / 0.1 \%$ TFA over $\left.30 \mathrm{~min}, t_{\mathrm{R}}=27.1 \mathrm{~min}\right)$.

\section{Methyl 3-Amino-5-(azepan-1-yl)-6-(2,6-dimethoxypyridin3-yl)-pyrazine-2-carboxylate (28a).}

General method A using 2,6-dimethoxypyridin-3-ylboronic acid (200 mg, $0.703 \mathrm{mmol}$ ) afforded $28 \mathrm{a}$ as a yellow solid; yield $42 \%(115 \mathrm{mg}, 0.297 \mathrm{mmol}) . \mathrm{Mp} 134-136{ }^{\circ} \mathrm{C} .{ }^{1} \mathrm{H}$ NMR $\left(500 \mathrm{MHz}, \mathrm{CDCl}_{3}\right) \delta 7.66(\mathrm{~d}, J=8.0 \mathrm{~Hz}, 1 \mathrm{H}), 6.38(\mathrm{~d}, J=8.0 \mathrm{~Hz}, 1 \mathrm{H}), 3.92(\mathrm{~s}, 3 \mathrm{H}), 3.87(\mathrm{~s}$, $3 \mathrm{H}), 3.86(\mathrm{~s}, 3 \mathrm{H}), 3.45$ (bs, 2H), 3.31 (bs, 2H), 1.57 (s, 4H), 1.44 (s, 4H). ${ }^{13} \mathrm{C}$ NMR $(126$ $\left.\mathrm{MHz}, \mathrm{CDCl}_{3}\right) \delta 167.5,162.8,159.7,155.3,153.9,141.5,127.6,116.2,112.2,101.3,53.7$, 53.5, 51.9, 50.1, 28.1, 27.1. HRESI-MS $m / z\left(\mathrm{M}+\mathrm{H}^{+}\right)$, Anal. for $\mathrm{C}_{19} \mathrm{H}_{25} \mathrm{~N}_{5} \mathrm{O}_{4}$ : calcd mass 388.1985. Found 388.1983. Anal. HPLC $\left(70: 30 \mathrm{H}_{2} \mathrm{O} / 0.1 \%\right.$ TFA/ $\mathrm{CH}_{3} \mathrm{CN} / 0.1 \%$ TFA $\rightarrow$ $100 \% \mathrm{CH}_{3} \mathrm{CN} / 0.1 \% \mathrm{TFA}$ over $\left.30 \mathrm{~min}, t_{\mathrm{R}}=31.6 \mathrm{~min}\right)$.

\section{Methyl 3-Amino-5-(azepan-1-yl)-6-(2,4-dimethoxyphenyl)-pyrazine-2-carboxylate (29a).}

General method A using 2,4-dimethoxyphenylboronic acid (128 mg, $0.702 \mathrm{mmol})$ afforded 29a as a yellow solid; yield 53\% (143 mg, $0.371 \mathrm{mmol})$. Mp 106-108 ${ }^{\circ} \mathrm{C} .{ }^{1} \mathrm{H}$ NMR (500 $\left.\mathrm{MHz}, \mathrm{CDCl}_{3}\right): \delta 7.33(\mathrm{~d}, J=8.3 \mathrm{~Hz}, 1 \mathrm{H}), 6.54(\mathrm{dd}, J=8.3 \mathrm{~Hz}, J=2.1 \mathrm{~Hz} 1 \mathrm{H}), 6.42(\mathrm{~d}, J=$ $2.1 \mathrm{~Hz}, 1 \mathrm{H}), 3.85(\mathrm{~s}, 3 \mathrm{H}), 3.82(\mathrm{~s}, 3 \mathrm{H}), 3.70(\mathrm{~s}, 3 \mathrm{H}), 3.44(\mathrm{~s}, 2 \mathrm{H}), 3.26(\mathrm{~s}, 2 \mathrm{H}), 1.58(\mathrm{~s}, 2 \mathrm{H})$, $1.53(\mathrm{~s}, 2 \mathrm{H}), 1.46(\mathrm{~s}, 2 \mathrm{H}), 1.39(\mathrm{~s}, 2 \mathrm{H}) .{ }^{13} \mathrm{C} \mathrm{NMR}\left(126 \mathrm{MHz}, \mathrm{CDCl}_{3}\right) \delta 166.0,160.9,158.2$, 155.4, 154.0, 131.1, 129.2, 123.7, 111.8, 105.1, 98.7, 55.7, 55.7, 52.0, 50.6, 27.8, 26.9. HRESI-MS $m / z\left(\mathrm{M}+\mathrm{Na}^{+}\right)$, Anal. For $\mathrm{C}_{20} \mathrm{H}_{27} \mathrm{~N}_{4} \mathrm{NaO}_{4}$ : calcd mass 409.1852. Found 409.1866. Anal. HPLC $\left(100 \mathrm{H}_{2} \mathrm{O} / 0.1 \% \mathrm{TFA} \rightarrow 100 \% \mathrm{CH}_{3} \mathrm{CN} / 0.1 \%\right.$ TFA over $30 \mathrm{~min}, t_{\mathrm{R}}=$ $28.6 \mathrm{~min})$. 
Methyl 3-Amino-5-(azepan-1-yl)-6-(3,5-dimethoxyphenyl)-pyrazine-2-carboxylate (30a).

General method A using 3,5-dimethoxyphenylboronic acid (128 mg, $0.702 \mathrm{mmol}$ ) afforded 30a as a yellow solid; 62\% (166 mg, $0.430 \mathrm{mmol})$. Mp 96-98 ${ }^{\circ} \mathrm{C} .{ }^{1} \mathrm{H}$ NMR $(500 \mathrm{MHz}$, $\left.\mathrm{CDCl}_{3}\right) \delta 6.64(\mathrm{~s}, 2 \mathrm{H}), 6.40(\mathrm{~s}, 1 \mathrm{H}), 3.88(\mathrm{~s}, 3 \mathrm{H}), 3.81(\mathrm{~s}, 6 \mathrm{H}), 3.38(\mathrm{t}, J=6.0 \mathrm{~Hz}, 4 \mathrm{H}), 1.64$ (s, 4H), $1.44(\mathrm{~s}, 4 \mathrm{H}) .{ }^{13} \mathrm{C} \mathrm{NMR}\left(126 \mathrm{MHz}, \mathrm{CDCl}_{3}\right) \delta 167.5,161.0,154.7,153.7,142.8$, 131.6, 112.0, 106.3, 100.0, 55.6, 52.0, 50.9, 28.1, 27.1. HRESI-MS $\mathrm{m} / \mathrm{z}\left(\mathrm{M}+\mathrm{Na}^{+}\right)$, Anal. for $\mathrm{C}_{20} \mathrm{H}_{26} \mathrm{~N}_{4} \mathrm{NaO}_{4}$ : calcd mass 409.1852. Found 409.1867. Anal. HPLC $\left(70: 30 \mathrm{H}_{2} \mathrm{O} / 0.1 \%\right.$ $\mathrm{TFA} / \mathrm{CH}_{3} \mathrm{CN} / 0.1 \% \mathrm{TFA} \rightarrow 100 \% \mathrm{CH}_{3} \mathrm{CN} / 0.1 \%$ TFA over $30 \mathrm{~min}, t_{\mathrm{R}}=30.0 \mathrm{~min}$ ).

\section{Methyl 3-Amino-5-(azepan-1-yl)-6-(3,6-dimethoxypyridazin-4-yl)pyrazine-2-carboxylate (31a).}

General method A using 3,6-dimethoxypyridazin-4-ylboronic acid (97 mg, $0.526 \mathrm{mmol}$ ) afforded 31a as a yellow solid; yield $68 \%$ (94 mg, $0.242 \mathrm{mmol}$ ). Mp 152-154 ${ }^{\circ} \mathrm{C} .{ }^{1} \mathrm{H}$ NMR $\left(500 \mathrm{MHz}, \mathrm{CDCl}_{3}\right) \delta 7.17(\mathrm{~s}, 1 \mathrm{H}), 4.08(\mathrm{~s}, 3 \mathrm{H}), 4.00(\mathrm{~s}, 3 \mathrm{H}), 3.88(\mathrm{~s}, 3 \mathrm{H}), 3.43(\mathrm{~s}, 2 \mathrm{H}), 3.29$ (s, 2H), 1.63 (s, 4H), $1.44(\mathrm{~s}, 4 \mathrm{H}) .{ }^{13} \mathrm{C}$ NMR $\left(126 \mathrm{MHz}, \mathrm{CDCl}_{3}\right) \delta 167.00,162.8,159.6$, 154.7, 154.1, 135.1, 122.9, 119.4, 113.1, 54.8, 54.6, 52.1, 49.7, 28.0, 27.2. HRESI-MS $\mathrm{m} / \mathrm{z}$ $\left(\mathrm{M}+\mathrm{Na}^{+}\right)$, Anal. for $\mathrm{C}_{18} \mathrm{H}_{24} \mathrm{~N}_{6} \mathrm{NaO}_{4}$ : calcd mass 411.1757. Found 411.1762. Anal. HPLC $\left(70: 30 \mathrm{H}_{2} \mathrm{O} / 0.1 \%\right.$ TFA/CH${ }_{3} \mathrm{CN} / 0.1 \% \mathrm{TFA} \rightarrow 100 \% \mathrm{CH}_{3} \mathrm{CN} / 0.1 \%$ TFA over $30 \mathrm{~min}, t_{\mathrm{R}}=$ $21.2 \mathrm{~min})$.

Methyl 3-Amino-6-(2-aminopyrimidin-5-yl)-5-(azepan-1-yl)-pyrazine-2-carboxylate (32a).

General method A using 2-aminopyrimidin-5-ylboronic acid (208 mg, $1.5 \mathrm{mmol})$ afforded 32a as a yellow solid; yield $83 \%$ (285 mg). Mp 216-218 ${ }^{\circ} \mathrm{C} .{ }^{1} \mathrm{H}$ NMR (500 MHz, DMSO$\left.d_{6}\right): \delta 8.27$ (s, 2H), $7.04\left(\mathrm{br} \mathrm{s}, 2 \mathrm{H}, \mathrm{NH}_{2}\right), 6.76\left(\mathrm{br} \mathrm{s}, 2 \mathrm{H}, \mathrm{NH}_{2}\right), 3.74(\mathrm{~s}, 3 \mathrm{H}), 3.41(\mathrm{t}, J=5.9$ $\mathrm{Hz}, 4 \mathrm{H}), 1.59$ (s, 4H), 1.41 (s, 4H). ${ }^{13} \mathrm{C}$ NMR (126 MHz, DMSO- $\left.d_{6}\right): \delta$ 27.0, 27.9, 51.0, $51.9,112.3,123.9,126.5,154.2,155.1,157.4,163.0,167.3,163.0,157.4,155.1,154.2$, 126.5, 123.9, 112.3, 51.9, 51.0, 27.9, 27.0. HRESI-MS $\mathrm{m} / \mathrm{z}\left(\mathrm{M}+\mathrm{H}^{+}\right)$, Anal. for $\mathrm{C}_{16} \mathrm{H}_{22} \mathrm{~N}_{7} \mathrm{O}_{2}$ : calcd mass 344.1835. Found 344.1833. Anal. HPLC $\left(100 \mathrm{H}_{2} \mathrm{O} / 0.1 \%\right.$ TFA $\rightarrow$ $100 \% \mathrm{CH}_{3} \mathrm{CN} / 0.1 \%$ TFA over $30 \mathrm{~min}, t_{\mathrm{R}}=14.2 \mathrm{~min}$ ).

\section{Methyl 3-Amino-5-(azepan-1-yl)-6-(2-chloropyrimidin-5-yl)-pyrazine-2-carboxylate (8).}

General method A using (2-chloropyrimidin-5-yl)boronic acid $(475 \mathrm{mg}, 3 \mathrm{mmol}$ ) afforded 8 as a yellow solid (460 mg, 64\%). Mp 168-170 ${ }^{\circ} \mathrm{C} .{ }^{1} \mathrm{H} \mathrm{NMR}\left(\mathrm{CDCl}_{3}\right): \delta 8.76(\mathrm{~s}, 2 \mathrm{H}), 6.13$ (br s, 2H, $\mathrm{NH}_{2}$ ), 3.91 (s, 3H), 3.35 (t, $\left.J=5.8 \mathrm{~Hz}, 4 \mathrm{H}\right), 1.71(\mathrm{~s}, 4 \mathrm{H}), 1.49$ (s, 4H). ${ }^{13} \mathrm{C} \mathrm{NMR}$ $\left(\mathrm{CDCl}_{3}\right): \delta 27.3,27.8,51.4,52.2,114.6,122.8,133.3,153.7,155.0,158.0,159.2,166.8$. MS (ESI): $m / z 363(\mathrm{M}+\mathrm{H})^{+}, 385(\mathrm{M}+\mathrm{H})^{+}$. HRESI-MS $\mathrm{m} / z\left(\mathrm{M}+\mathrm{H}^{+}\right)$, Anal. for $\mathrm{C}_{16} \mathrm{H}_{20} \mathrm{ClN}_{6} \mathrm{O}_{2}$ : calcd mass 363.1336. Found 363.1347. Anal. HPLC $\left(100 \mathrm{H}_{2} \mathrm{O} / 0.1 \%\right.$ TFA $\rightarrow 100 \% \mathrm{CH}_{3} \mathrm{CN} / 0.1 \%$ TFA over $\left.30 \mathrm{~min}, t_{\mathrm{R}}=17.6 \mathrm{~min}\right)$.

\section{General Method B. Synthesis of Methyl 3-Amino-5-(azepan1-yl)-6-(2-(substituted amino)pyrimidin-5-yl)pyrazine-2-carboxylates 33a and 34a.}

To a solution of methyl 3-amino-5-(azepan-1-yl)-6-(2-chloropyrimidin-5-yl)pyrazine-2carboxylate $8(0.25 \mathrm{mmol})$ in DMF $(2 \mathrm{~mL})$ at room temperature was added the appropriate amine $(0.75 \mathrm{mmol})$. DIPEA $(0.75 \mathrm{mmol})$ was then added, and the reaction mixture was 
stirred at room temperature for 2 days. The mixture was extracted with EtOAc $(3 \times 10 \mathrm{~mL})$, and the combined organic layer was washed with brine, dried $\left(\mathrm{MgSO}_{4}\right)$, and evaporated. The residue was purified by silica gel flash column chromatography using EtOAc/pet. spirit.

Methyl 3-Amino-5-(azepan-1-yl)-6-(2-(methylamino)-pyrimidin-5-yl)pyrazine-2-carboxylate (33a).

General method B using 8 (91 mg, $0.25 \mathrm{mmol})$ and methylamine ( $2 \mathrm{M}$ in $\mathrm{MeOH}, 0.375 \mathrm{~mL}$, $0.75 \mathrm{mmol}$ ) gave $33 \mathrm{a}$ as a yellow solid; yield $87 \%(78.0 \mathrm{mg}) . \mathrm{Mp} 158-160{ }^{\circ} \mathrm{C} .{ }^{1} \mathrm{H}$ NMR $\left(500 \mathrm{MHz}, \mathrm{CDCl}_{3}\right): \delta 8.45(\mathrm{~s}, 2 \mathrm{H}), 6.22\left(\mathrm{br} \mathrm{s}, 2 \mathrm{H}, \mathrm{NH}_{2}\right), 5.28(\mathrm{q}, J=4.9 \mathrm{~Hz}, 1 \mathrm{H}, \mathrm{NH}), 3.90$ (s, 3H), $3.42(\mathrm{t}, J=5.9 \mathrm{~Hz}, 4 \mathrm{H}), 3.04(\mathrm{~d}, J=5.1 \mathrm{~Hz}, 3 \mathrm{H}), 1.67(\mathrm{~s}, 4 \mathrm{H}), \delta 1.47(\mathrm{~s}, 4 \mathrm{H}) .{ }^{13} \mathrm{C}$ NMR (126 MHz, $\left.\mathrm{CDCl}_{3}\right): \delta 167.4,162.0,157.2,155.3,153.7,126.9,124.2,113.3,52.3$, 51.3, 28.8, 28.0, 27.3. HRESI-MS $m / z\left(\mathrm{M}+\mathrm{H}^{+}\right)$, Anal. for $\mathrm{C}_{17} \mathrm{H}_{24} \mathrm{~N}_{7} \mathrm{O}_{2} 358.1991$, found 358.2001. Anal. HPLC $\left(100 \mathrm{H}_{2} \mathrm{O} / 0.1 \% \mathrm{TFA} \rightarrow 100 \% \mathrm{CH}_{3} \mathrm{CN} / 0.1 \%\right.$ TFA over $30 \mathrm{~min}, \mathrm{t}_{\mathrm{R}}=$ $14.8 \mathrm{~min})$.

\section{Methyl 3-Amino-5-(azepan-1-yl)-6-(2-(isopropylamino)-pyrimidin-5-yl)pyrazine-2- carboxylate (34a).}

General method B using 8 (91 $\mathrm{mg}, 0.25 \mathrm{mmol})$ and isopropylamine $(45 \mathrm{mg}, 0.75 \mathrm{mmol})$ gave 34a as a yellow solid; yield $83 \%$ (80 mg). Mp $155-157{ }^{\circ} \mathrm{C} .{ }^{1} \mathrm{H}$ NMR $(500 \mathrm{MHz}$, $\mathrm{CDCl}_{3}$ ): $\delta 8.43(\mathrm{~s}, 2 \mathrm{H}), 6.25(\mathrm{br} \mathrm{s}, 2 \mathrm{H}), 5.10(\mathrm{~d}, J=7.8 \mathrm{~Hz}, 1 \mathrm{H}), 4.16$ (septet, $J=6.8 \mathrm{~Hz}$, $1 \mathrm{H}), 3.89$ (s, 3H), 3.43 (t, $J=5.9 \mathrm{~Hz}, 4 \mathrm{H}), 1.67$ (s, 4H), 1.47 (s, 4H), 1.25 (d, J = 7.0 Hz, $6 \mathrm{H}) .{ }^{13} \mathrm{C} \mathrm{NMR}\left(126 \mathrm{MHz}, \mathrm{CDCl}_{3}\right): \delta 167.2,157.0,155.1,153.5,126.8,123.8,113.1,52.0$, 51.1, 43.0, 27.8, 27.1, 22.9. HRESI-MS $m / z\left(\mathrm{M}+\mathrm{H}^{+}\right)$, Anal. for for $\mathrm{C}_{19} \mathrm{H}_{28} \mathrm{~N}_{7} \mathrm{O}_{2} 386.2304$, found 386.2318. Anal. HPLC $\left(100 \mathrm{H}_{2} \mathrm{O} / 0.1 \%\right.$ TFA $\rightarrow 100 \% \mathrm{CH}_{3} \mathrm{CN} / 0.1 \%$ TFA over 30 $\left.\min , t_{\mathrm{R}}=16.1 \mathrm{~min}\right)$.

\section{Methyl 3-Amino-5-(azepan-1-yl)-6-(2-(2-hydroxyethyl)-amino)pyrimidin-5-yl)pyrazine-2- carboxylate (35a).}

To a solution of methyl 3-amino-5-(azepan-1-yl)-6-(2-chloropyrimidin-5-yl)-pyrazine-2carboxylate $8(91 \mathrm{mg}, 0.25 \mathrm{mmol})$ in 2-propanol $(5 \mathrm{~mL})$ was added ethanolamine $(16.8 \mathrm{mg}$, $0.017 \mathrm{~mL}, 0.275 \mathrm{mmol}$ ). DIPEA ( $35.5 \mathrm{mg}, 0.048 \mathrm{~mL}, 0.275 \mathrm{mmol}$ ) was then added, and the reaction mixture was heated at reflux for $2 \mathrm{~h}$. After cooling to room temperature, additional equivalents of ethanolamine $(0.275 \mathrm{mmol})$ and DIPEA $(0.275 \mathrm{mmol})$ were added, and the reaction mixture was heated for a further $2 \mathrm{~h}$. The mixture was allowed to cool to room temperature overnight, which caused the product to crystallize. The solid product was collected by vacuum filtration and washed with cold 2-propanol $(2 \times 2 \mathrm{~mL})$ and diethyl ether $(2 \mathrm{~mL}$ ). After drying, the pure product was obtained as light yellow crystals ( $82 \mathrm{mg}, 84 \%$ ). Mp 164-166 ${ }^{\circ} \mathrm{C}^{1} \mathrm{H}$ NMR $\left(500 \mathrm{MHz}, \mathrm{CDCl}_{3}\right): \delta, 8.44(\mathrm{~s}, 2 \mathrm{H}), 6.23$ (br s, 2H), $5.64(\mathrm{t}, J=$ $5.5 \mathrm{~Hz}, 1 \mathrm{H}), 3.90(\mathrm{~s}, 3 \mathrm{H}), 3.85(\mathrm{t}, J=4.4 \mathrm{~Hz}, 2 \mathrm{H}), 3.62(\mathrm{q}, J=4.9 \mathrm{~Hz}, 2 \mathrm{H}), 3.42(\mathrm{t}, J=5.6$ $\mathrm{Hz}, 4 \mathrm{H}), 1.68$ (s, 4H), 1.48 (s, 4H). ${ }^{13} \mathrm{C}$ NMR $\left(126 \mathrm{MHz}, \mathrm{CDCl}_{3}\right): \delta 167.1,161.7,156.9$, 155.1, 153.5, 126.2, 124.6, 113.3, 63.6, 52.1, 51.1, 44.9, 27.8, 27.1. HRESI-MS $m / z(\mathrm{M}+\mathrm{H}$ $\left.{ }^{+}\right)$, Anal. For $\mathrm{C}_{18} \mathrm{H}_{26} \mathrm{~N}_{7} \mathrm{O}_{3} 388.2097$, found 388.2088. Anal. HPLC $\left(100 \mathrm{H}_{2} \mathrm{O} / 0.1 \%\right.$ TFA $\rightarrow$ $100 \% \mathrm{CH}_{3} \mathrm{CN} / 0.1 \%$ TFA over $30 \mathrm{~min}, t_{\mathrm{R}}=14.4 \mathrm{~min}$ ). 


\section{General Method C. Synthesis of Acyl Guanidines Using the Reported Method.}

${ }^{38}$ Free guanidine was generated via neutralization of guanidine $\cdot \mathrm{HCl}$ with $\mathrm{Na}^{+}{ }^{+} \mathrm{PrO}^{-}$in i PrOH to create a 2 -fold molar excess relative to the 6-substituted pyrazinoyl methyl ester. A stock solution of ${ }^{\mathrm{i}} \mathrm{PrO}^{-}$was prepared by adding $\mathrm{Na}$ (typically $30-70 \mathrm{mg}$ ) in anhydrous ${ }^{i} \mathrm{PrOH}$ under $\mathrm{N}_{2}$ and heated at $70{ }^{\circ} \mathrm{C}$ for $30 \mathrm{~min}$. From this stock solution an amount of ${ }^{i} \mathrm{PrO}$ - was removed via syringe and made up to $10 \mathrm{~mL}$ in anhydrous ${ }^{i} \mathrm{PrOH}$. To this solution was added a stoichiometric amount of guanidine hydrochloride, and the mixture was stirred at $70{ }^{\circ} \mathrm{C}$ for $30 \mathrm{~min}$. This solution was gravity filtered onto the neat 6 -substituted pyrazinoyl methyl ester. Once filtration was complete, the mixture was heated to reflux and allowed to react for $1-6 \mathrm{~h}$. The reactions were monitored by MS and TLC and terminated by removal of solvent under reduced pressure. Final products were isolated by rp-HPLC. All compounds were isolated as TFA salts and exchanged to their respective $\mathrm{HCl}$ salts by stirring with quaternary ammonium $\mathrm{Cl}^{-}$anion exchange resin in $\mathrm{MeOH}$ prior to biological evaluation.

\section{General Method D. Improved Synthesis of Acyl Guanidines.}

Dry $\mathrm{CH}_{3} \mathrm{OH}(50 \mathrm{~mL})$ was slowly added with stirring to small pieces of sodium ( $2.3 \mathrm{~g}, 0.1$ mol) cooled on ice under nitrogen. After complete dissolution of the sodium, guanidine hydrochloride $(9.55 \mathrm{~g}, 0.1 \mathrm{~mol}$ ) was added and the resulting mixture was stirred at room temperature for $1.5 \mathrm{~h}$. Filtration of the white precipitate gave a $2 \mathrm{M}$ solution of free guanidine in methanol, which was collected in a sealed dry flask under nitrogen and stored in a refrigerator.

To a suspension of the requisite methyl ester (1 equiv, $0.2-0.5 \mathrm{mmol}$ ) in DMF ( $5 \mathrm{~mL}$ ) was added $2 \mathrm{M}$ guanidine in methanol (10 equiv, 2-5 mmol), and the resulting mixture was stirred at room temperature overnight. Brine $(20 \mathrm{~mL})$ was added and the mixture extracted with EtOAc $(3 \times 30 \mathrm{~mL})$. The organic layer was washed with $10 \% \mathrm{NaCl}(2 \times 30 \mathrm{~mL})$, dried $\left(\mathrm{MgSO}_{4}\right)$, and concentrated under reduced pressure. The crude mixture was dissolved in 5 $\mathrm{mL}$ of $30 \% \mathrm{CH}_{3} \mathrm{CN} / 70 \% \mathrm{H}_{2} \mathrm{O}$ and $0.1 \%$ acetic acid and loaded onto a reverse phase silica gel chromatography column. Isocratic elution was performed with $\mathrm{CH}_{3} \mathrm{CN} / \mathrm{H}_{2} \mathrm{O}$ mixtures containing $0.1 \%$ acetic acid. The pooled fractions containing pure compound by TLC analysis were concentrated under reduced pressure and neutralized with solid $\mathrm{NaHCO}_{3}$. The aqueous mixture was extracted with EtOAc $(3 \times 30 \mathrm{~mL})$, and the combined organic layer was washed with brine, dried $\left(\mathrm{MgSO}_{4}\right)$, and evaporated to give the free acylguanidine.

\section{3-Amino-5-(azepan-1-yl)-N-carbamimidoyl-6-(4-(methylthio)phenyl)pyrazine-2-carboxamide} (10).

General method C using 10a (70 mg, $0.214 \mathrm{mmol}$ ) gave 10 as a yellow solid; yield 5\% (6 $\mathrm{mg}, 0.015 \mathrm{mmol}) . \mathrm{Mp} 152-154{ }^{\circ} \mathrm{C} .{ }^{1} \mathrm{H}$ NMR $\left(500 \mathrm{MHz}, \mathrm{CDCl}_{3}\right) \delta 10.31(\mathrm{~s}, 1 \mathrm{H}), 9.44(\mathrm{~s}$, 2H), 8.47 (s, 2H), $7.44(\mathrm{~d}, J=8.1 \mathrm{~Hz}, 2 \mathrm{H}), 7.28(\mathrm{~d}, J=8.1 \mathrm{~Hz}, 2 \mathrm{H}), 3.40(\mathrm{t}, J=5.5 \mathrm{~Hz}, 4 \mathrm{H})$ $1.60(\mathrm{~s}, 4 \mathrm{H}), 1.46$ (s, 4H). ${ }^{13} \mathrm{C}$ NMR $\left(126 \mathrm{MHz}, \mathrm{CDCl}_{3}\right) \delta 166.0,156.6,155.7,153.6,139.0$, 136.4, 131.2, 128.1, 126.6, 111.0, 51.5, 27.9, 27.1, 15.5. HRESIMS $\mathrm{m} / \mathrm{z}\left(\mathrm{M}+\mathrm{H}^{+}\right)$Anal. for $\mathrm{C}_{19} \mathrm{H}_{26} \mathrm{~N}_{7} \mathrm{OS}$ : calcd mass 400.1920. Found 400.1935. Anal. HPLC. (70:30 $\mathrm{H}_{2} \mathrm{O} / 0.1 \%$ $\mathrm{TFA} / \mathrm{CH}_{3} \mathrm{CN} / 0.1 \% \mathrm{TFA} \rightarrow 100 \% \mathrm{CH}_{3} \mathrm{CN} / 0.1 \%$ TFA over $\left.30 \mathrm{~min}, t_{\mathrm{R}}=28.4 \mathrm{~min}\right)$. 


\section{3-Amino-5-(azepan-1-yl)-N-carbamimidoyl-6-(4-(trifluoromethyl)phenyl)pyrazine-2- carboxamide (11).}

General method D using 11a (394 mg, $1.0 \mathrm{mmol}$ ) gave 11 as a yellow solid; yield $92 \%$ (385 mg). Mp 172-175 ${ }^{\circ} \mathrm{C} .{ }^{1} \mathrm{H}$ NMR $\left(\mathrm{CD}_{3} \mathrm{OD}\right): \delta 7.68(\mathrm{~d}, J=8.0 \mathrm{~Hz}, 2 \mathrm{H}), 7.65(\mathrm{~d}, J=8.0 \mathrm{~Hz}$, $2 \mathrm{H}), 3.38(\mathrm{t}, J=5.0 \mathrm{~Hz}, 4 \mathrm{H}), 1.65(\mathrm{~s}, 4 \mathrm{H}), 1.46(\mathrm{~s}, 4 \mathrm{H}) .{ }^{13} \mathrm{C} \mathrm{NMR}\left(\mathrm{CD}_{3} \mathrm{OD}\right): \delta 167.9$, 160.2, 155.8, 155.0, 145.6, 130.1, 130.0 (d, J = $32 \mathrm{~Hz}), 129.2,125.8(\mathrm{~d}, \mathrm{~J}=2.7 \mathrm{~Hz}), 124.3$ (d, $J=170), 112.9,51.6,28.6,27.6$. HRESI-MS $m / z\left(\mathrm{M}+\mathrm{H}^{+}\right)$Anal. for $\mathrm{C}_{19} \mathrm{H}_{23} \mathrm{~F}_{3} \mathrm{~N}_{7} \mathrm{O}$ : calcd mass 422.1916. Found 422.1911. Anal. HPLC $\left(100 \% \mathrm{H}_{2} \mathrm{O} / 0.1 \%\right.$ TFA $\rightarrow 100 \%$ $\mathrm{CH}_{3} \mathrm{CN} / 0.1 \%$ TFA over $30 \mathrm{~min}, t_{\mathrm{R}}=27.9 \mathrm{~min}$ ).

\section{3-Amino-5-(azepan-1-yl)-6-(benzo[d][1,3]dioxol-5-yl)-N-carbamimidoylpyrazine-2- carboxamide (12).}

General method $\mathrm{C}$ using $12 \mathrm{a}(54 \mathrm{mg}, 0.146 \mathrm{mmol}$ ) gave 12 as a yellow oil; yield $9 \%$ (6 mg, $0.015 \mathrm{mmol}$ ). Mp $154-156{ }^{\circ} \mathrm{C} .{ }^{1} \mathrm{H}$ NMR $\left(500 \mathrm{MHz}, \mathrm{DMSO}-d_{6}\right) \delta 10.39(\mathrm{~s}, 1 \mathrm{H}), 8.71(\mathrm{~s}$, $2 \mathrm{H}), 8.27(\mathrm{~s}, 2 \mathrm{H}), 7.31(\mathrm{~s}, 1 \mathrm{H}), 6.96(\mathrm{~m}, 2 \mathrm{H}), 6.08(\mathrm{~s}, 2 \mathrm{H}), 3.40(\mathrm{t}, J=5.75 \mathrm{~Hz}, 4 \mathrm{H}), 1.60(\mathrm{~s}$, $2 \mathrm{H}), 1.39$ (s, 2H). ${ }^{13} \mathrm{C}$ NMR $\left(126 \mathrm{MHz}, \mathrm{DMSO}-d_{6}\right) \delta 166.2,155.2,154.8,153.6,147.26$, 146.7, 133.4, 130.3, 121.4, 112.7, 108.5, 108.0, 101.2, 50.4, 27.2, 26.1. HRESI-MS $\mathrm{m} / \mathrm{z}(\mathrm{M}$ $+\mathrm{H}^{+}$) Anal. for $\mathrm{C}_{19} \mathrm{H}_{24} \mathrm{~N}_{7} \mathrm{O}_{3}$ : calcd mass 398.1941. Found 398.1929. Anal. HPLC (70:30 $\mathrm{H}_{2} \mathrm{O} / 0.1 \% \mathrm{TFA} / \mathrm{CH}_{3} \mathrm{CN} / 0.1 \% \mathrm{TFA} \rightarrow 100 \% \mathrm{CH}_{3} \mathrm{CN} / 0.1 \%$ TFA over $30 \mathrm{~min}, t_{\mathrm{R}}=26.4$ $\min )$.

\section{3-Amino-5-(azepan-1-yl)-N-carbamimidoyl-6-(isoquinolin-4-yl)pyrazine-2-carboxamide (13).}

General method C using 13a (156 mg, $0.413 \mathrm{mmol}$ ) gave 13 as an orange solid; yield $15 \%$ (32 mg, $0.079 \mathrm{mmol}$ ). Mp 158-160 ${ }^{\circ} \mathrm{C} .{ }^{1} \mathrm{H}$ NMR $\left(500 \mathrm{MHz}, \mathrm{CD}_{3} \mathrm{OD}\right) \delta 10.47$ (s, exchange with solvent), 9.57 (bs, 1H), 9.19 (s, exchange with solvent), 9.09 (s, exchange with solvent) 8.69 (bs, $1 \mathrm{H}), 8.40$ (d, $J=8.2 \mathrm{~Hz}, 1 \mathrm{H}), 8.01$ (t, $J=7.5 \mathrm{~Hz}, 1 \mathrm{H}), 7.91$ (t, $J=7.5 \mathrm{~Hz}, 1 \mathrm{H}), 7.81$ (d, $J=8.2 \mathrm{~Hz}, 1 \mathrm{H}), 3.37$ (s, 2H), 3.23 (s, 2H), 1.53 (s, 4H), 1.44 (s, 4H). ${ }^{13} \mathrm{C}$ NMR (126 $\left.\mathrm{MHz}, \mathrm{CD}_{3} \mathrm{OD}\right) \delta 167.5,157.7,156.9,156.4,149.7,137.6,136.9,136.9,136.8,131.6,131.4$, 129.0, 125.7, 124.7, 113.0, 51.6, 29.0, 27.8. HRESI-MS $m / z\left(\mathrm{M}+\mathrm{H}^{+}\right)$Anal. for $\mathrm{C}_{20} \mathrm{H}_{24} \mathrm{~N}_{7} \mathrm{OS}$ : calcd mass 410.1763 . Found 410.1758. Anal. HPLC $\left(70: 30 \mathrm{H}_{2} \mathrm{O} / 0.1 \%\right.$ TFA/ $\mathrm{CH}_{3} \mathrm{CN} / 0.1 \% \mathrm{TFA} \rightarrow 100 \% \mathrm{CH}_{3} \mathrm{CN} / 0.1 \%$ TFA over $\left.30 \mathrm{~min}, t_{\mathrm{R}}=22.7 \mathrm{~min}\right)$.

\section{3-Amino-5-(azepan-1-yl)-N-carbamimidoyl-6-(1-methyl-1 H-pyrazol-4-yl)pyrazine-2- carboxamide (14).}

General method $\mathrm{C}$ using $14 \mathrm{a}(69 \mathrm{mg}, 0.207 \mathrm{mmol}$ ) gave 14 as a yellow oil; yield $38 \%$ (32 $\mathrm{mg}, 0.193 \mathrm{mmol}) . \mathrm{Mp} 150-152{ }^{\circ} \mathrm{C} .{ }^{1} \mathrm{H} \mathrm{NMR}\left(500 \mathrm{MHz}, \mathrm{CDCl}_{3}\right) \delta 10.49(\mathrm{~s}, 1 \mathrm{H}), 8.83(\mathrm{~s}$, $2 \mathrm{H}), 8.52(\mathrm{~s}, 2 \mathrm{H}), 8.00(\mathrm{~s}, 1 \mathrm{H}), 7.57(\mathrm{~s}, 1 \mathrm{H}), 5.49(\mathrm{bs}, 2 \mathrm{H}), 3.93(\mathrm{~s}, 3 \mathrm{H}), 3.50(\mathrm{t}, J=5.75 \mathrm{~Hz}$, $4 \mathrm{H}), 1.69(\mathrm{~s}, 4 \mathrm{H}), 1.49(\mathrm{~s}, 4 \mathrm{H}) .{ }^{13} \mathrm{C} \mathrm{NMR}\left(126 \mathrm{MHz}, \mathrm{CDCl}_{3}\right) \delta 166.6,156.4,156.1,153.4$, 137.4, 130.7, 125.1, 121.7, 111.1, 51.5, 38.7, 27.9, 27.3. HRESI-MS $m / z\left(\mathrm{M}+\mathrm{H}^{+}\right)$Anal. for $\mathrm{C}_{16} \mathrm{H}_{24} \mathrm{~N}_{9} \mathrm{O}$ : calcd mass 358.2104. Found 358.2118. Anal. HPLC $\left(70: 30 \mathrm{H}_{2} \mathrm{O} / 0.1 \%\right.$ $\mathrm{TFA} / \mathrm{CH}_{3} \mathrm{CN} / 0.1 \% \mathrm{TFA} \rightarrow 100 \% \mathrm{CH}_{3} \mathrm{CN} / 0.1 \%$ TFA over $30 \mathrm{~min}, t_{\mathrm{R}}=21.0 \mathrm{~min}$ ). 


\section{3-Amino-5-(azepan-1-yl)-N-carbamimidoyl-6-(1-(2-morpholinoethyl)-1H-pyrazol-4- yl)pyrazine-2-carboxamide (15).}

General method C using 15a (99 mg, $0.229 \mathrm{mmol}$ ) gave 15 as an orange oil; yield $20 \%$ (22 $\mathrm{mg}, 0.048 \mathrm{mmol}) . \mathrm{Mp} 157-159^{\circ} \mathrm{C} .{ }^{1} \mathrm{H}$ NMR $\left(500 \mathrm{MHz}, \mathrm{CDCl}_{3}\right) \delta 10.48(\mathrm{~s}, 1 \mathrm{H}), 8.86(\mathrm{~s}$, 2H), $8.65(\mathrm{~s}, 2 \mathrm{H}), 7.89(\mathrm{~s}, 1 \mathrm{H}), 7.56(\mathrm{~s}, 1 \mathrm{H}), 4.67(\mathrm{~s}, 2 \mathrm{H}), 3.93(\mathrm{~s}, 4 \mathrm{H}), 3.69(\mathrm{~s}, 2 \mathrm{H}),$,3.47 (s, $4 \mathrm{H}), 3.42(\mathrm{bs}, 2 \mathrm{H}), 3.05(\mathrm{bs}, 2 \mathrm{H}) ,1.67(\mathrm{~s}, 4 \mathrm{H}), 1.49(\mathrm{~s}, 4 \mathrm{H}) .{ }^{13} \mathrm{C} \mathrm{NMR}\left(126 \mathrm{MHz}, \mathrm{CDCl}_{3}\right) \delta$ 166.6, 156.4, 156.1, 153.6, 140.0, 130.6, 124.1, 122.9, 111.3, 63.8, 57.2, 53.3, 51.4, 46.4, 27.9, 27.2. HRESI-MS $m / z\left(\mathrm{M}+\mathrm{H}^{+}\right)$Anal. for $\mathrm{C}_{21} \mathrm{H}_{33} \mathrm{~N}_{10} \mathrm{O}_{2}$ : calcd mass 457.2788. Found 457.2796. Anal. HPLC (70:30 $\mathrm{H}_{2} \mathrm{O} / 0.1 \%$ TFA/CH${ }_{3} \mathrm{CN} / 0.1 \% \mathrm{TFA} \rightarrow 100 \% \mathrm{CH}_{3} \mathrm{CN} / 0.1 \%$ TFA over $\left.30 \mathrm{~min}, t_{\mathrm{R}}=19.0 \mathrm{~min}\right)$.

\section{3-Amino-5-(azepan-1-yl)-N-carbamimidoyl-6-(1H-indol-2-yl)pyrazine-2-carboxamide (16).}

General method C using 16a (76 mg, $0.209 \mathrm{mmol}$ ) gave 16 as an orange solid; yield $31 \%$ (29 mg, $0.074 \mathrm{mmol}) . \mathrm{Mp} 152-154{ }^{\circ} \mathrm{C} .{ }^{1} \mathrm{H}$ NMR $\left(500 \mathrm{MHz}, \mathrm{CDCl}_{3}\right) \delta 11.09(\mathrm{~s}, 1 \mathrm{H}), 10.82$ (s, 1H), $8.82(\mathrm{~s}, 2 \mathrm{H}), 7.92(\mathrm{~s}, 2 \mathrm{H}), 7.55(\mathrm{~d}, 1 \mathrm{H}, J=7.7 \mathrm{~Hz}), 7.41(\mathrm{~d}, 1 \mathrm{H}, J=7.7 \mathrm{~Hz}), 7.12(\mathrm{t}$, $1 \mathrm{H}, J=7 \mathrm{~Hz}), 7.03(\mathrm{t}, 1 \mathrm{H}), 6.44(\mathrm{~s}, 1 \mathrm{H}), 3.64(\mathrm{t}, J=5.0 \mathrm{~Hz}, 4 \mathrm{H}), 1.73(\mathrm{~s}, 4 \mathrm{H}), 1.50(\mathrm{~s}, 4 \mathrm{H})$. ${ }^{13} \mathrm{C} \mathrm{NMR}\left(126 \mathrm{MHz}, \mathrm{CDCl}_{3}\right) \delta 166.7,156.2,155.7,153.0,136.6,134.9,128.3,125.7$, 122.7, 120.5, 119.8, 111.7, 110.8, 102.3, 52.1, 27.9, 27.6. HRESI-MS m/z $\left(\mathrm{M}+\mathrm{H}^{+}\right)$Anal. for $\mathrm{C}_{20} \mathrm{H}_{28} \mathrm{~N}_{7} \mathrm{O}_{3}$ : calcd mass 393.2151. Found 393.2159. Anal. HPLC $\left(70: 30 \mathrm{H}_{2} \mathrm{O} / 0.1 \%\right.$ $\mathrm{TFA} / \mathrm{CH}_{3} \mathrm{CN} / 0.1 \% \mathrm{TFA} \rightarrow 100 \% \mathrm{CH}_{3} \mathrm{CN} / 0.1 \%$ TFA over $\left.30 \mathrm{~min}, t_{\mathrm{R}}=24.4 \mathrm{~min}\right)$.

\section{3-Amino-5-(azepan-1-yl)-6-(benzo[b]thiophen-2-yl)-N-carbamimidoylpyrazine-2- carboxamide (17).}

General method D using 17a (383 mg, $1.0 \mathrm{mmol}$ ) gave 17 as a yellow solid; yield $78 \%$ (320 $\mathrm{mg}$ ). Mp 220-224 ${ }^{\circ} \mathrm{C} .{ }^{1} \mathrm{H}$ NMR (500 MHz, $\left.\mathrm{CDCl}_{3}\right) \delta 10.48(\mathrm{~s}, 1 \mathrm{H}), 8.92(\mathrm{~s}, 2 \mathrm{H}), 8.76(\mathrm{~s}$, $2 \mathrm{H}), 7.60(\mathrm{~d}, J=7.5 \mathrm{~Hz}, 1 \mathrm{H}), 7.43(\mathrm{~d}, J=7.5 \mathrm{~Hz}, 1 \mathrm{H}), 7.31(\mathrm{t}, J=7.5 \mathrm{~Hz}, 1 \mathrm{H}), 7.26(\mathrm{t}, J=$ $7.5 \mathrm{~Hz}, 1 \mathrm{H}), 7.20(\mathrm{~s}, 1 \mathrm{H}), 3.50(\mathrm{t}, J=5.75 \mathrm{~Hz}, 4 \mathrm{H}), 1.69(\mathrm{~s}, 4 \mathrm{H}), 1.49(\mathrm{~s}, 4 \mathrm{H}) .{ }^{13} \mathrm{C} \mathrm{NMR}$ $\left(126 \mathrm{MHz}, \mathrm{CDCl}_{3}\right) \delta$ 168.2. 164.9, 160.7, 156.5, 155.4, 144.1, 141.3, 141.2, 125.8, 125.5, 124.7, 123.5, 123.0, 113.5, 52.0, 29.2, 28.1. HRESI-MS $m / z\left(\mathrm{M}+\mathrm{H}^{+}\right) \mathrm{C}_{20} \mathrm{H}_{24} \mathrm{~N}_{7} \mathrm{OS}$ : calcd mass 410.1763. Found 410.1758. Anal. HPLC $\left(100 \% \mathrm{H}_{2} \mathrm{O} / 0.1 \%\right.$ TFA $\rightarrow 100 \% \mathrm{CH}_{3} \mathrm{CN} /$ $0.1 \%$ TFA over $30 \mathrm{~min}, t_{\mathrm{R}}=27.5 \mathrm{~min}$ ).

\section{3-Amino-5-(azepan-1-yl)-6-(benzofuran-2-yl)-N-carbamimidoylpyrazine-2-carboxamide (18).}

General method D using 18a (366 mg, $1.0 \mathrm{mmol}$ ) gave 18 as a yellow solid; yield $86 \%$ (338 $\mathrm{mg})$. Mp 130-132 ${ }^{\circ} \mathrm{C} ;{ }^{1} \mathrm{H}$ NMR (CD $\left.{ }_{3} \mathrm{OD}\right): \delta 7.64(\mathrm{~d}, \mathrm{~J}=7.5 \mathrm{~Hz}, 1 \mathrm{H}), 7.50(\mathrm{~d}, J=8.0 \mathrm{~Hz}$, $1 \mathrm{H}), 7.31(\mathrm{t}, J=8.0 \mathrm{~Hz}, 1 \mathrm{H}), 7.26(\mathrm{t}, J=7.5 \mathrm{~Hz}, 1 \mathrm{H}), 7.05(\mathrm{~s}, 1 \mathrm{H}), 3.51(\mathrm{t}, J=5.0 \mathrm{~Hz}, 4 \mathrm{H})$, $1.71(\mathrm{~s}, 4 \mathrm{H}), 1.54(\mathrm{~s}, 4 \mathrm{H}) .{ }^{13} \mathrm{C} \mathrm{NMR}\left(\mathrm{CD}_{3} \mathrm{OD}\right): \delta 167.4,164.3,154.6,154.5,154.4,154.1$, 129.7, 124.9, 123.7, 121.6, 121.1, 112.4, 111.5, 105.6, 50.3, 28.6, 27.3. HRESI-MS $m / z$ (M $+\mathrm{H}^{+}$) Anal. for $\mathrm{C}_{20} \mathrm{H}_{24} \mathrm{~N}_{7} \mathrm{O}_{2}$ : calcd mass 394.1991. Found 394.1986. Anal. HPLC (100\% $\mathrm{H}_{2} \mathrm{O} / 0.1 \% \mathrm{TFA} \rightarrow 100 \% \mathrm{CH}_{3} \mathrm{CN} / 0.1 \%$ TFA over $\left.30 \mathrm{~min}, t_{\mathrm{R}}=27.5 \mathrm{~min}\right)$. 


\section{3-Amino-5-(azepan-1-yl)-N-carbamimidoyl-6-(4-fluoro-benzofuran-2-yl)pyrazine-2- carboxamide (19).}

General method C using 19a (54 mg, $0.139 \mathrm{mmol}$ ) gave 19 as an orange solid; yield $26 \%$ (15 mg, $0.036 \mathrm{mmol}$ ). Mp 132-136 ${ }^{\circ} \mathrm{C} .{ }^{1} \mathrm{H}$ NMR (500 MHz, $\left.\mathrm{CD}_{3} \mathrm{OD}\right) \delta 10.47$ (s, exchange with solvent), 8.98 (s, exchange with solvent), 8.33 (s, exchange with solvent), 7.36 (t, $J=$ $8.7 \mathrm{~Hz}, 1 \mathrm{H}), 7.32(\mathrm{q}, J=5.5 \mathrm{~Hz}, 1 \mathrm{H}), 7.22(\mathrm{~s}, 1 \mathrm{H}), 7.02(\mathrm{t}, J=8.7 \mathrm{~Hz}, 1 \mathrm{H}), 3.44(\mathrm{t}, J=5.8$ $\mathrm{Hz}, 4 \mathrm{H}) 1.72(\mathrm{~s}, 4 \mathrm{H}), 1.51(\mathrm{~s}, 4 \mathrm{H}) .{ }^{13} \mathrm{C}$ NMR $\left(126 \mathrm{MHz}, \mathrm{CD}_{3} \mathrm{OD}\right) \delta 167.3,158.2,157.4$, 157.0, 156.9, 156.2, 156.2, 155.7, 126.6, 121.2, 112.2, 109.8, 108.7, 102.4, 51.1, 29.0, 27.9. HRESI-MS $m / z\left(\mathrm{M}+\mathrm{H}^{+}\right)$Anal. for $\mathrm{C}_{20} \mathrm{H}_{22} \mathrm{FN}_{7} \mathrm{O}_{2}$, : calcd mass 412.1897. Found 412.1909. Anal. HPLC $\left(70: 30 \mathrm{H}_{2} \mathrm{O} / 0.1 \%\right.$ TFA/ $\mathrm{CH}_{3} \mathrm{CN} / 0.1 \%$ TFA $\rightarrow 100 \% \mathrm{CH}_{3} \mathrm{CN} / 0.1 \%$ TFA over $\left.30 \mathrm{~min}, t_{\mathrm{R}}=25.6 \mathrm{~min}\right)$.

\section{3-Amino-5-(azepan-1-yl)-N-carbamimidoyl-6-(thiophen-2-yl)pyrazine-2-carboxamide (20).}

General method $\mathrm{C}$ using 20a (99 mg, $0.298 \mathrm{mmol}$ ) gave 20 as a yellow oil; yield $2 \%$ ( $2 \mathrm{mg}$, $0.006 \mathrm{mmol}) . \mathrm{Mp} 136-138{ }^{\circ} \mathrm{C} .{ }^{1} \mathrm{H}$ NMR $\left(500 \mathrm{MHz}, \mathrm{CDCl}_{3}\right) \delta 10.10$ (s, 1H), 10.04 (bs, 2H), $8.08(\mathrm{bs}, 2 \mathrm{H}), 7.26(\mathrm{~d}, J=5 \mathrm{~Hz}, 1 \mathrm{H}), 7.03(\mathrm{~d}, J=3 \mathrm{~Hz}, 1 \mathrm{H}), 3.47(\mathrm{t}, J=5.75 \mathrm{~Hz}, 4 \mathrm{H}), 1.68$ $(\mathrm{m}, 4 \mathrm{H}), 1.49(\mathrm{~m}, 4 \mathrm{H}) .{ }^{13} \mathrm{C} \mathrm{NMR}\left(126 \mathrm{MHz}, \mathrm{CDCl}_{3}\right) \delta 167.0,156.3,156.1,153.4,139.9$, 128.4, 128.2, 125.2, 124.5, 111.0, 51.0, 27.8, 27.1. HRESI-MS $\mathrm{m} / \mathrm{z}\left(\mathrm{M}+\mathrm{H}^{+}\right)$Anal. for $\mathrm{C}_{16} \mathrm{H}_{21} \mathrm{~N}_{7} \mathrm{O}$ : calcd mass 360.1607. Found 360.1610. Anal. HPLC (70:30 $\mathrm{H}_{2} \mathrm{O} / 0.1 \%$ $\mathrm{TFA} / \mathrm{CH}_{3} \mathrm{CN} / 0.1 \% \mathrm{TFA} \rightarrow 100 \% \mathrm{CH}_{3} \mathrm{CN} / 0.1 \%$ TFA over $30 \mathrm{~min}, t_{\mathrm{R}}=24.2 \mathrm{~min}$ ).

\section{3-Amino-5-(azepan-1-yl)-N-carbamimidoyl-6-(thiophen-3-yl)pyrazine-2-carboxamide (21).}

General method C using 21a (103 mg, $0.309 \mathrm{mmol}$ ) gave 21 as an orange oil; yield 17\% (24 $\mathrm{mg}, 0.067 \mathrm{mmol}) . \mathrm{Mp} 138-140{ }^{\circ} \mathrm{C} .{ }^{1} \mathrm{H} \mathrm{NMR}\left(500 \mathrm{MHz}, \mathrm{CDCl}_{3}\right) \delta 10.31(\mathrm{~s}, 1 \mathrm{H}), 8.62$ (bs, $2 \mathrm{H}), 8.29$, (bs, 2H), $7.78(\mathrm{~m}, 3 \mathrm{H}), 7.64(\mathrm{dd}, J=8.5,1.5 \mathrm{~Hz}, 1 \mathrm{H}$ ), $7.44(\mathrm{~m}, 2 \mathrm{H}), 6.23$ (bs, $2 \mathrm{H}), 3.38(\mathrm{t}, J=5.75,4 \mathrm{H}), 1.59(\mathrm{~s}, 4 \mathrm{H}), 1.42(\mathrm{~s}, 4 \mathrm{H}) .{ }^{13} \mathrm{C} \mathrm{NMR}\left(126 \mathrm{MHz}, \mathrm{CDCl}_{3}\right) \delta 166.7$, 156.2, 156.0, 153.6, 140.1, 128.4, 127.8, 125.9, 123.6, 110.7, 51.3, 27.9, 27.1. HRESIMS $\mathrm{m} / \mathrm{z}\left(\mathrm{M}+\mathrm{H}^{+}\right)$Anal. for $\mathrm{C}_{16} \mathrm{H}_{21} \mathrm{~N}_{7} \mathrm{O}$ : calcd mass 360.1607. Found 360.1611. Anal. HPLC $\left(70: 30 \mathrm{H}_{2} \mathrm{O} / 0.1 \% \mathrm{TFA} / \mathrm{CH}_{3} \mathrm{CN} / 0.1 \% \mathrm{TFA} \rightarrow 100 \% \mathrm{CH}_{3} \mathrm{CN} / 0.1 \%\right.$ TFA over $30 \mathrm{~min}, t_{\mathrm{R}}=$ $25.2 \mathrm{~min})$.

\section{3-Amino-5-(azepan-1-yl)-N-carbamimidoyl-6-(furan-2-yl)-pyrazine-2-carboxamide (22).}

General method D using 22a (316 mg, $1.0 \mathrm{mmol}$ ) gave 22 as a yellow solid; yield $91 \%$ (310 $\mathrm{mg})$. Mp 143-146 ${ }^{\circ} \mathrm{C} .{ }^{1} \mathrm{H}$ NMR $\left(\mathrm{CD}_{3} \mathrm{OD}\right): \delta 7.50(\mathrm{~s}, 1 \mathrm{H}), 6.55(\mathrm{~d}, J=3.0 \mathrm{~Hz}, 1 \mathrm{H}), 6.50(\mathrm{~s}$, $1 \mathrm{H}), 3.37(\mathrm{t}, J=6.0 \mathrm{~Hz}, 4 \mathrm{H}), 1.64(\mathrm{~s}, 4 \mathrm{H}), 1.47(\mathrm{~s}, 4 \mathrm{H}) .{ }^{13} \mathrm{C}$ NMR $\left(\mathrm{CD}_{3} \mathrm{OD}\right): \delta 168.1,160.7$, 156.3, 156.0, 153.4, 142.8, 122.4, 112.6, 112.4, 109.9, 50.9, 29.2, 27.8. HRESI-MS m/z (M $+\mathrm{H}^{+}$) Anal. for $\mathrm{C}_{16} \mathrm{H}_{22} \mathrm{~N}_{7} \mathrm{O}_{2}$ : calcd mass 344.1835. Found 344.1830. Anal. HPLC (100\% $\mathrm{H}_{2} \mathrm{O} / 0.1 \% \mathrm{TFA} \rightarrow 100 \% \mathrm{CH}_{3} \mathrm{CN} / 0.1 \%$ TFA over $\left.30 \mathrm{~min}, t_{\mathrm{R}}=24.3 \mathrm{~min}\right)$.

\section{3-Amino-5-(azepan-1-yl)-N-carbamimidoyl-6-(furan-3-yl)-pyrazine-2-carboxamide (23).}

General method C using 23a (90 mg, $0.285 \mathrm{mmol}$ ) gave 23 as a yellow solid; yield 35\% (41 $\mathrm{mg}, 0.119 \mathrm{mmol}) . \mathrm{Mp} 146-148{ }^{\circ} \mathrm{C} .{ }^{1} \mathrm{H} \mathrm{NMR}\left(500 \mathrm{MHz}, \mathrm{CDCl}_{3}\right) \delta 10.42(\mathrm{~s}, 1 \mathrm{H}), 8.54(\mathrm{~s}$, $4 \mathrm{H}), 7.63(\mathrm{~s}, 1 \mathrm{H}), 7.42(\mathrm{~s}, 1 \mathrm{H}), 6.63(\mathrm{~s}, 1 \mathrm{H}), 5.35(\mathrm{bs}, 2 \mathrm{H}), 3.52(\mathrm{t}, J=5.75 \mathrm{~Hz}, 4 \mathrm{H}), 1.68$ (s, 4H), 1.49 (s, 4H). $\left.{ }^{13} \mathrm{C} \mathrm{NMR} \mathrm{(126} \mathrm{MHz,} \mathrm{CDCl}_{3}\right) \delta 166.4,156.3,155.8,153.6,143.2,141.1$, 
125.3, 124.9, 110.8, 110.7, 51.4, 27.9, 27.1. HRESI-MS $m / z\left(\mathrm{M}+\mathrm{H}^{+}\right)$Anal. for $\mathrm{C}_{16} \mathrm{H}_{22} \mathrm{~N}_{7} \mathrm{O}_{2}$ : calcd mass 344.1835. Found 344.1837. Anal. HPLC $\left(70: 30 \mathrm{H}_{2} \mathrm{O} / 0.1 \%\right.$ TFA/ $\mathrm{CH}_{3} \mathrm{CN} / 0.1 \% \mathrm{TFA} \rightarrow 100 \% \mathrm{CH}_{3} \mathrm{CN} / 0.1 \%$ TFA over $\left.30 \mathrm{~min}, t_{\mathrm{R}}=25.5 \mathrm{~min}\right)$.

3-Amino-5-(azepan-1-yl)-N-carbamimidoyl-6-(2-fluoropyridin-3-yl)pyrazine-2-carboxamide (24).

General method C using 24a (100 mg, $0.290 \mathrm{mmol}$ ) gave 24 as a yellow solid; yield $13 \%$ (18 $\mathrm{mg}, 0.048 \mathrm{mmol}) . \mathrm{Mp} 160-162{ }^{\circ} \mathrm{C} .{ }^{1} \mathrm{H}$ NMR $\left(500 \mathrm{MHz}, \mathrm{CDCl}_{3}\right) \delta 10.42(\mathrm{~s}, 1 \mathrm{H}), 8.59$ (bs, $4 \mathrm{H}), 8.17(\mathrm{~m}, 2 \mathrm{H}), 7.29(\mathrm{t}, J=5.4 \mathrm{~Hz}) 5.13(\mathrm{bs}, 2 \mathrm{H}), 3.38(\mathrm{~s}, 4 \mathrm{H}), 1.64(\mathrm{~s}, 4 \mathrm{H}), 1.47$ (s, 4H). ${ }^{13} \mathrm{C}$ NMR $\left(126 \mathrm{MHz}, \mathrm{CDCl}_{3}\right) \delta 166.5,160.7,158.8,156.0,155.9,154.0,146.9,141.4$, 124.2, 123.4, 122.5, 111.8, 50.4, 27.9, 27.2. HRESI-MS $\mathrm{m} / \mathrm{Z}\left(\mathrm{M}+\mathrm{H}^{+}\right)$Anal. for $\mathrm{C}_{17} \mathrm{H}_{22} \mathrm{FN}_{8} \mathrm{O}$ : calcd mass 373.1901. Found 373.1916. Anal. HPLC (70:30 $\mathrm{H}_{2} \mathrm{O} / 0.1 \%$ $\mathrm{TFA} / \mathrm{CH}_{3} \mathrm{CN} / 0.1 \% \mathrm{TFA} \rightarrow 100 \% \mathrm{CH}_{3} \mathrm{CN} / 0.1 \%$ TFA over $30 \mathrm{~min}, t_{\mathrm{R}}=22.2 \mathrm{~min}$ ).

\section{3-Amino-5-(azepan-1-yl)-N-carbamimidoyl-6-(pyrimidin-5-yl)pyrazine-2-carboxamide (25).}

General method D using 25a (328 mg, $1.0 \mathrm{mmol}$ ) gave 25 as a yellow solid; yield $87 \%$ (305 $\mathrm{mg}) . \mathrm{Mp} 188-190{ }^{\circ} \mathrm{C} .{ }^{1} \mathrm{H}$ NMR $\left(\mathrm{CD}_{3} \mathrm{OD}\right): \delta 8.98(\mathrm{~s}, 1 \mathrm{H}), 8.97(\mathrm{~s}, 2 \mathrm{H}), 3.35(\mathrm{t}, J=5.5 \mathrm{~Hz}$, $4 \mathrm{H}), 1.67(\mathrm{~s}, 4 \mathrm{H}), 1.47(\mathrm{~s}, 4 \mathrm{H}) .{ }^{13} \mathrm{C} \mathrm{NMR}\left(\mathrm{CD}_{3} \mathrm{OD}\right): \delta 162.2,156.5,156.3,155.7,155.5$, 154.0, 153.5, 135.2, 122.2, 51.0, 28.0, 27.1. HRESI-MS $m / z\left(\mathrm{M}+\mathrm{H}^{+}\right)$. Anal. for $\mathrm{C}_{16} \mathrm{H}_{22} \mathrm{~N}_{9} \mathrm{O}$ : calcd mass 356.1947. Found 356.1942. Anal. HPLC (70:30 $\mathrm{H}_{2} \mathrm{O} / 0.1 \%$ $\mathrm{TFA} / \mathrm{CH}_{3} \mathrm{CN} / 0.1 \% \mathrm{TFA} \rightarrow 100 \% \mathrm{CH}_{3} \mathrm{CN} / 0.1 \%$ TFA over $\left.30 \mathrm{~min}, t_{\mathrm{R}}=20.1 \mathrm{~min}\right)$.

\section{3-Amino-5-(azepan-1-yl)-N-carbamimidoyl-6-(2-methoxypyrimidin-5-yl)pyrazine-2- carboxamide (26).}

General method D using 26a (358 mg, $1.0 \mathrm{mmol}$ ) gave 26 as a yellow solid; yield $90 \%$ (345 $\mathrm{mg}) . \mathrm{Mp} 148-150{ }^{\circ} \mathrm{C} .{ }^{1} \mathrm{H}$ NMR $\left(\mathrm{CD}_{3} \mathrm{OD}\right): \delta 8.77(\mathrm{~s}, 2 \mathrm{H}), 4.08(\mathrm{~s}, 3 \mathrm{H}), 3.46(\mathrm{t}, J=5.5 \mathrm{~Hz}$, $4 \mathrm{H}), 1.72(\mathrm{~s}, 4 \mathrm{H}), 1.54(\mathrm{~s}, 4 \mathrm{H}) .{ }^{13} \mathrm{C}$ NMR $\left(\mathrm{CD}_{3} \mathrm{OD}\right): \delta 166.7,164.1,163.4,158.1,154.8$, 153.6, 128.6, 124.2, 112.8, 54.3, 27.7, 26.7. HRESI-MS $m / z\left(\mathrm{M}+\mathrm{H}^{+}\right)$Anal. for $\mathrm{C}_{17} \mathrm{H}_{24} \mathrm{~N}_{9} \mathrm{O}_{2}$ : calcd mass 386.2053 . Found 386.2048. Anal. HPLC $\left(100 \% \mathrm{H}_{2} \mathrm{O} / 0.1 \%\right.$ TFA $\rightarrow 100 \% \mathrm{CH}_{3} \mathrm{CN} / 0.1 \%$ TFA over $30 \mathrm{~min}, t_{\mathrm{R}}=20.7 \mathrm{~min}$ ).

\section{3-Amino-5-(azepan-1-yl)-N-carbamimidoyl-6-(2,4-dimethoxypyrimidin-5-yl)pyrazine-2- carboxamide (27).}

General method C using 27a (104 mg, $0.267 \mathrm{mmol}$ ) gave 27 as a yellow solid; yield 54\% (65 $\mathrm{mg}, 0.156 \mathrm{mmol}) . \mathrm{Mp} 184-186^{\circ} \mathrm{C} .{ }^{1} \mathrm{H}$ NMR $\left(\mathrm{CD}_{3} \mathrm{OD}\right) \delta 10.76(\mathrm{~s}, 1 \mathrm{H}), 9.27(\mathrm{~s}, 2 \mathrm{H}), 8.51$ (s, 2H), 8.48 (s, 2H), 4.08 (s, 3H), $3.35(\mathrm{t}, J=5.75 \mathrm{~Hz}, 4 \mathrm{H}), 1.66(\mathrm{~s}, 4 \mathrm{H}), 1.46(\mathrm{~s}, 4 \mathrm{H}) .{ }^{13} \mathrm{C}$ NMR $\left(126 \mathrm{MHz}, \mathrm{CDCl}_{3}\right) \delta 166.6,164.0,157.8,156.1,153.9,127.8,124.7,112.5,55.6$, 51.6, 27.8, 27.1. HRESI-MS $m / z\left(\mathrm{M}+\mathrm{H}^{+}\right)$Anal. for $\mathrm{C}_{18} \mathrm{H}_{26} \mathrm{~N}_{9} \mathrm{O}_{3}$ : calcd mass 416.2159. Found 416.2158. Anal. HPLC (70:30 $\mathrm{H}_{2} \mathrm{O} / 0.1 \%$ TFA/ $\mathrm{CH}_{3} \mathrm{CN} / 0.1 \% \mathrm{TFA} \rightarrow 100 \% \mathrm{CH}_{3} \mathrm{CN} /$ $0.1 \%$ TFA over $\left.30 \mathrm{~min}, t_{\mathrm{R}}=21.4 \mathrm{~min}\right)$. 


\section{3-Amino-5-(azepan-1-yl)-N-carbamimidoyl-6-(2,6-dimethoxypyridin-3-yl)pyrazine-2- carboxamide (28).}

General method C using $28 \mathrm{a}$ ( $98 \mathrm{mg}, 0.253 \mathrm{mmol}$ ) gave 28 as a yellow solid; yield $13 \%$ (15 $\mathrm{mg}, 0.036 \mathrm{mmol}) . \mathrm{Mp} 152-154{ }^{\circ} \mathrm{C} .{ }^{1} \mathrm{H}$ NMR $\left(500 \mathrm{MHz}, \mathrm{CDCl}_{3}\right) \delta 10.28(\mathrm{~s}, 1 \mathrm{H}), 8.77(\mathrm{~s}$, $2 \mathrm{H}), 8.14(\mathrm{~s}, 2 \mathrm{H}), 7.61(\mathrm{~d}, J=8.0 \mathrm{~Hz}), 6.39$ (d, $J=8.0 \mathrm{~Hz}, 1 \mathrm{H}), 3.94(\mathrm{~s}, 3 \mathrm{H}), 3.87$ (s, 3H), $3.52(\mathrm{~s}, 2 \mathrm{H}), 3.35(\mathrm{~s}, 2 \mathrm{H}), 1.60(\mathrm{~s}, 4 \mathrm{H}), 1.51(\mathrm{~s}, 2 \mathrm{H}), 1.44(\mathrm{~s}, 2 \mathrm{H}) .{ }^{13} \mathrm{C} \mathrm{NMR}(126 \mathrm{MHz}$, $\left.\mathrm{CDCl}_{3}\right) \delta 166.0,163.2,159.2,155.4,153.5,141.1,128.0,114.6,110.2,101.3,53.8,53.5$, 50.4, 27.7, 26.81. HRESI-MS $\mathrm{m} / \mathrm{z}\left(\mathrm{M}+\mathrm{H}^{+}\right)$Anal. for $\mathrm{C}_{19} \mathrm{H}_{27} \mathrm{~N}_{8} \mathrm{O}_{3}$ : calcd mass 415.2206. Found 415.2191. Anal. HPLC (70:30 $\mathrm{H}_{2} \mathrm{O} / 0.1 \%$ TFA/CH ${ }_{3} \mathrm{CN} / 0.1 \%$ TFA $\rightarrow 100 \% \mathrm{CH}_{3} \mathrm{CN} /$ $0.1 \%$ TFA over $\left.30 \mathrm{~min}, t_{\mathrm{R}}=26.1 \mathrm{~min}\right)$.

\section{3-Amino-5-(azepan-1-yl)-N-carbamimidoyl-6-(2,4-dimethoxyphenyl)pyrazine-2-carboxamide (29).}

General method $\mathrm{C}$ using $29 \mathrm{a}(123 \mathrm{mg}, 0.317 \mathrm{mmol})$ gave 29 as a yellow solid; yield $8 \%$ (12 $\mathrm{mg}, 0.029 \mathrm{mmol}) . \mathrm{Mp} 156-158{ }^{\circ} \mathrm{C} .{ }^{1} \mathrm{H} \mathrm{NMR}\left(500 \mathrm{MHz}, \mathrm{CDCl}_{3}\right) \delta 10.19(\mathrm{~s}, 1 \mathrm{H}), 9.10(\mathrm{bs}$, 2H), $7.79(\mathrm{bs}, 2 \mathrm{H}), 7.23(\mathrm{~d}, J=8.1 \mathrm{~Hz}), 6.58(\mathrm{~d}, J=8.1 \mathrm{~Hz}), 6.48(\mathrm{~s}, 1 \mathrm{H}) 3.86(\mathrm{~s}, 3 \mathrm{H}), 3.74$ $(\mathrm{s}, 3 \mathrm{H}), 3.51(\mathrm{~s}, 2 \mathrm{H}), 3.36(\mathrm{~s}, 2 \mathrm{H}), 1.59(\mathrm{~s}, 4 \mathrm{H}), 1.50(\mathrm{~s}, 2 \mathrm{H}), 1.45(\mathrm{~s}, 2 \mathrm{H}) .{ }^{13} \mathrm{C} \mathrm{NMR}(126$ $\left.\mathrm{MHz}, \mathrm{CDCl}_{3}\right) \delta 166.0,161.5,157.8,155.7,155.4,153.8,130.6,129.6,122.1,109.8,105.0$, 98.7, 55.7, 55.6, 50.6, 27.8, 26.9. HRESI-MS $m / z\left(\mathrm{M}+\mathrm{H}^{+}\right)$Anal. for $\mathrm{C}_{20} \mathrm{H}_{28} \mathrm{~N}_{7} \mathrm{O}_{3}$ : calcd mass 414.2254. Found 414.2269. Anal. HPLC (70:30 $\mathrm{H}_{2} \mathrm{O} / 0.1 \%$ TFA/CH${ }_{3} \mathrm{CN} / 0.1 \% \mathrm{TFA} \rightarrow$ $100 \% \mathrm{CH}_{3} \mathrm{CN} / 0.1 \%$ TFA over $\left.30 \mathrm{~min}, t_{\mathrm{R}}=26.7 \mathrm{~min}\right)$.

\section{3-Amino-5-(azepan-1-yl)-N-carbamimidoyl-6-(3,5-dimethoxyphenyl)pyrazine-2-carboxamide (30).}

General method C using 30a (147 mg, $0.381 \mathrm{mmol}$ ) gave 30 as a yellow solid; yield $26 \%$ (44 $\mathrm{mg}, 0.116 \mathrm{mmol}) . \mathrm{Mp} 138-140^{\circ} \mathrm{C} .{ }^{1} \mathrm{H} \mathrm{NMR}\left(500 \mathrm{MHz}, \mathrm{CDCl}_{3}\right) \delta 10.75(\mathrm{~s}, 1 \mathrm{H}), 8.81(\mathrm{bs}$, $2 \mathrm{H}), 8.60(\mathrm{bs}, 2 \mathrm{H}), 6.73(\mathrm{~d}, J=2.2 \mathrm{~Hz}), 6.58(\mathrm{t}, J=2.2 \mathrm{~Hz}, 1 \mathrm{H}), 3.84(\mathrm{~s}, 6 \mathrm{H}), 3.38(\mathrm{~s}, 2 \mathrm{H})$, $1.64(\mathrm{~s}, 4 \mathrm{H}), 1.45$ (s, 2H). ${ }^{13} \mathrm{C} \mathrm{NMR}\left(126 \mathrm{MHz}, \mathrm{CDCl}_{3}\right) \delta 167.0,160.1,156.4,155.6,153.8$, 141.6, 132.9, 110.9, 106.5, 100.3, 55.9, 51.0, 28.0, 27.1. HRESI-MS $\mathrm{m} / \mathrm{z}\left(\mathrm{M}+\mathrm{H}^{+}\right)$Anal. for $\mathrm{C}_{20} \mathrm{H}_{28} \mathrm{~N}_{7} \mathrm{O}_{3}$ : calcd mass 414.2254. Found 414.2244. Anal. HPLC $\left(70: 30 \mathrm{H}_{2} \mathrm{O} / 0.1 \%\right.$ $\mathrm{TFA} / \mathrm{CH}_{3} \mathrm{CN} / 0.1 \% \mathrm{TFA} \rightarrow 100 \% \mathrm{CH}_{3} \mathrm{CN} / 0.1 \%$ TFA over $\left.30 \mathrm{~min}, t_{\mathrm{R}}=25.8 \mathrm{~min}\right)$.

\section{3-Amino-5-(azepan-1-yl)-N-carbamimidoyl-6-(3,6-dimethoxypyridazin-4-yl)pyrazine-2- carboxamide (31).}

General method C using 31a (94 mg, $0.243 \mathrm{mmol}$ ) gave 31 as a yellow solid; yield $17 \%$ ( 21 $\mathrm{mg}, 0.051 \mathrm{mmol}) . \mathrm{Mp} 202-204{ }^{\circ} \mathrm{C} .{ }^{1} \mathrm{H}$ NMR $\left(500 \mathrm{MHz}, \mathrm{CD}_{3} \mathrm{OD}\right) \delta 10.38$ (s, exchange with solvent), 8.77 (s, exchange with solvent), 8.21 (s, exchange with solvent), 7.35 (s, 1H), 4.06 $(\mathrm{s}, 3 \mathrm{H}), 3.99(\mathrm{~s}, 3 \mathrm{H}), 3.35(\mathrm{~s}, 4 \mathrm{H}), 1.66(\mathrm{~s}, 4 \mathrm{H}), 1.55(\mathrm{~s}, 2 \mathrm{H}), 1.45(\mathrm{~s}, 2 \mathrm{H}) .{ }^{13} \mathrm{C}$ NMR $(126$ $\left.\mathrm{MHz}, \mathrm{CD}_{3} \mathrm{OD}\right) \delta 167.3,164.1,160.9,156.9,156.8,156.1,136.3,124.0,121.4,112.2,55.4$, 55.3, 51.0, 28.9, 27.9. HRESI-MS $m / z\left(\mathrm{M}+\mathrm{H}^{+}\right)$Anal. for $\mathrm{C}_{18} \mathrm{H}_{26} \mathrm{~N}_{9} \mathrm{O}_{3}$ : calcd mass 416.2159. Found 416.2150. Anal. HPLC (70:30 $\mathrm{H}_{2} \mathrm{O} / 0.1 \%$ TFA/CH${ }_{3} \mathrm{CN} / 0.1 \%$ TFA $\rightarrow 00 \%$ $\mathrm{CH}_{3} \mathrm{CN} / 0.1 \%$ TFA over $\left.30 \mathrm{~min}, t_{\mathrm{R}}=19.0 \mathrm{~min}\right)$. 


\section{3-Amino-6-(2-aminopyrimidin-5-yl)-5-(azepan-1-yl)-N-carbamimidoylpyrazine-2- carboxamide (32).}

General method D using 32a ( $86 \mathrm{mg}, 0.25 \mathrm{mmol}$ ) gave 32 as a yellow solid; yield $78 \%$ (72 mg). Mp 225-228; ${ }^{1} \mathrm{H}$ NMR (DMSO-d6): $\delta 1.41$ (s, 4H), 1.60 (s, 4H), 3.38 (t, $J=5.9 \mathrm{~Hz}$, $4 \mathrm{H}$ ), 6.73 (br s, 2H), 7.25 (br s, $1 \mathrm{H}), 8.40$ (s, 2H). ${ }^{13} \mathrm{C}$ NMR (DMSO- $\left.d_{6}\right): \delta 167.5,162.9$, 159.1, 157.5, 154.9, 153.7, 126.0, 123.6, 112.9, 51.1, 28.0, 27.0. HRESI-MS $m / z\left(\mathrm{M}+\mathrm{H}^{+}\right)$, Anal. for $\mathrm{C}_{16} \mathrm{H}_{23} \mathrm{~N}_{10} \mathrm{O}_{3}$ : calcd mass 371.2056. Found 371.2067. Anal. HPLC $\left(100 \% \mathrm{H}_{2} \mathrm{O} /\right.$ $0.1 \% \mathrm{TFA} \rightarrow 100 \% \mathrm{CH}_{3} \mathrm{CN} / 0.1 \%$ TFA over $\left.30 \mathrm{~min}, t_{\mathrm{R}}=10.2 \mathrm{~min}\right)$.

\section{3-Amino-5-(azepan-1-yl)-N-carbamimidoyl-6-(2-(methylamino)pyrimidin-5-yl)pyrazine-2- carboxamide (33).}

General method D using 33a (39 mg, $0.11 \mathrm{mmol}$ ) gave 33 as a yellow solid; yield $85 \%$ (36 $\mathrm{mg}$ ). Mp 168-170; ${ }^{1} \mathrm{H}$ NMR (CD $\left.\mathrm{CD}\right): \delta 8.47$ (s, 2H), $3.48(\mathrm{t}, J=5.6 \mathrm{~Hz}, 4 \mathrm{H}), 2.97(\mathrm{~s}, 3 \mathrm{H})$, $1.69(\mathrm{~s}, 4 \mathrm{H}), 1.51(\mathrm{~s}, 4 \mathrm{H}) .{ }^{13} \mathrm{C} \mathrm{NMR}\left(\mathrm{CD}_{3} \mathrm{OD}\right): \delta 28.0,28.5,29.0,52.0,113.5,124.4,127.3$, $155.4,156.7,158.3,158.5,162.9,168.3,162.9,158.5,158.3,156.7,155.4,127.3,124.4$, 113.5, 52.0, 29.0, 28.5, 28.0. HRESI-MS $m / z\left(\mathrm{M}+\mathrm{H}^{+}\right)$Anal. for $\mathrm{C}_{17} \mathrm{H}_{25} \mathrm{~N}_{10} \mathrm{O}$ : calcd mass 385.2213. Found 385.2195. Anal. HPLC $\left(100 \% \mathrm{H}_{2} \mathrm{O} / 0.1 \% \mathrm{TFA} \rightarrow 100 \% \mathrm{CH}_{3} \mathrm{CN} / 0.1 \%\right.$ TFA over $30 \mathrm{~min}, t_{\mathrm{R}}=14.8 \mathrm{~min}$ ).

\section{3-Amino-5-(azepan-1-yl)-N-carbamimidoyl-6-(2-(isopropylamino)pyrimidin-5-yl)pyrazine-2- carboxamide (34).}

General method D using 34a (35 mg, $0.09 \mathrm{mmol}$ ) gave 34 as a yellow solid; yield $89 \%$ (32 $\mathrm{mg}$ ). Mp 172-175 ${ }^{\circ} \mathrm{C} .{ }^{1} \mathrm{H}$ NMR $\left(\mathrm{CD}_{3} \mathrm{OD}\right): \delta 8.46(\mathrm{~s}, 2 \mathrm{H}), 4.14$ (septet, $\mathrm{J}=6.8 \mathrm{~Hz}, 1 \mathrm{H}$ ), $3.47(\mathrm{t}, J=5.6 \mathrm{~Hz}, 4 \mathrm{H}), 1.69(\mathrm{~s}, 4 \mathrm{H}), 1.51(\mathrm{~s}, 4 \mathrm{H}), 1.25(\mathrm{~d}, J=6.5 \mathrm{~Hz}, 6 \mathrm{H}) .{ }^{13} \mathrm{C} \mathrm{NMR}$ $\left(\mathrm{CD}_{3} \mathrm{OD}\right): \delta 21.6,26.8,27.8,42.8,50.9,112.3,123.2,126.2,154.2,155.5,157.1,158.8$, 160.6, 167.1, 160.6, 158.8, 157.1, 155.5, 154.2, 126.2, 123.3, 112.3, 50.9, 42.8, 27.8, 26.8, 21.6. HRESI-MS $m / z\left(\mathrm{M}+\mathrm{H}^{+}\right)$Anal. for $\mathrm{C}_{19} \mathrm{H}_{29} \mathrm{~N}_{10} \mathrm{O}$ : calcd mass 413.2526. Found 413.2525. Anal. HPLC $\left(100 \% \mathrm{H}_{2} \mathrm{O} / 0.1 \% \mathrm{TFA} \rightarrow 100 \% \mathrm{CH}_{3} \mathrm{CN} / 0.1 \%\right.$ TFA over $30 \mathrm{~min}, t_{\mathrm{R}}$ $=16.1 \mathrm{~min})$.

\section{3-Amino-5-(azepan-1-yl)-N-carbamimidoyl-6-(2-((2-hydroxyethyl)amino)pyrimidin-5- yl)pyrazine-2-carboxamide (35).}

General method D using 35a (50 mg, $0.13 \mathrm{mmol}$ ) gave 35 as a Yellow solid: yield $86 \%$ (46 $\mathrm{mg})$. Mp 188-191 ${ }^{\circ} \mathrm{C} .{ }^{1} \mathrm{H}$ NMR $\left(\mathrm{CD}_{3} \mathrm{OD}\right): \delta 8.48(\mathrm{~s}, 2 \mathrm{H}), 3.72(\mathrm{t}, J=5.6 \mathrm{~Hz}, 2 \mathrm{H})$ ), $3.54(\mathrm{t}$, $J=5.6 \mathrm{~Hz}, 2 \mathrm{H}), 3.45$ (t, $J=5.8 \mathrm{~Hz}, 4 \mathrm{H}), 1.68(\mathrm{~s}, 4 \mathrm{H}), 1.50$ (s, 4H). ${ }^{13} \mathrm{C} \mathrm{NMR}\left(\mathrm{CD}_{3} \mathrm{OD}\right): \delta$ $167.1,162.3,160.8,158.2,156.3,154.9,126.5,124.7,117.5,61.7,51.9,44.6,28.9,27.8$. HRESI-MS $m / z\left(\mathrm{M}+\mathrm{H}^{+}\right)$Anal. for $\mathrm{C}_{18} \mathrm{H}_{27} \mathrm{~N}_{10} \mathrm{O}_{2}$ : calcd mass 415.2318. Found 415.2324. Anal. HPLC $\left(100 \% \mathrm{H}_{2} \mathrm{O} / 0.1 \%\right.$ TFA $\rightarrow 100 \% \mathrm{CH}_{3} \mathrm{CN} / 0.1 \%$ TFA over $30 \mathrm{~min}, t_{\mathrm{R}}=14.4$ $\min )$.

\section{Methyl 3-((Di-tert-butyloxycarbonyl)amino)-5-(azepan-1-yl)-6-chloro-2-pyrazinecarboxylate (36). \\ To a stirred solution of $7(2.85 \mathrm{~g}, 10 \mathrm{mmol})$ and DMAP $(61 \mathrm{mg}, 0.5 \mathrm{mmol})$ in 1,2dichloroethane $(40 \mathrm{~mL})$ was added a solution of di-tert-butyl dicarbonate $(6.57 \mathrm{~g}, 30$}


mmol) in 1,2-dichloroethane $(20 \mathrm{~mL})$ at $\mathrm{rt}$. The reaction mixture was heated at $70{ }^{\circ} \mathrm{C}$ for 18 $\mathrm{h}$. After cooling to room temperature, additional equivalents of di-tert-butyl dicarbonate $(3.28 \mathrm{~g}, 15 \mathrm{mmol})$ and DIPEA $(10 \mathrm{~mL})$ were added and the reaction mixture was heated for a further $18 \mathrm{~h}$. Solvents were evaporated and the residue was partitioned between EtOAc $(100 \mathrm{~mL})$ and water $(100 \mathrm{~mL})$. The aqueous layer was extracted with EtOAc $(3 \times 75 \mathrm{~mL})$, and the combined organic layer was washed with brine, dried $\left(\mathrm{MgSO}_{4}\right)$, and concentrated. The residue was purified by silica gel flash column chromatography using EtOAc/pet. spirit $(10-15 \%)$ to give $36(4.10 \mathrm{~g}, 85 \%)$ as a white solid. $\mathrm{Mp} 116-118^{\circ} \mathrm{C} .{ }^{1} \mathrm{H} \mathrm{NMR}\left(\mathrm{CDCl}_{3}\right): \delta$ $3.89(\mathrm{~s}, 3 \mathrm{H}), 3.88(\mathrm{t}, J=6.1 \mathrm{~Hz}, 4 \mathrm{H}), 1.55(\mathrm{~s}, 4 \mathrm{H}), 1.84(\mathrm{~s}, 4 \mathrm{H}), 1.41(\mathrm{~s}, 18 \mathrm{H}) .{ }^{13} \mathrm{C} \mathrm{NMR}$ $\left(\mathrm{CDCl}_{3}\right): \delta 163.0,152.3,150.4,145.4,128.6,126.1,83.3,52.8,51.6,28.1,28.1,26.7 . \mathrm{MS}$ (ESI): $m / z 507(\mathrm{M}+\mathrm{Na})^{+}$. HRESI-MS $\mathrm{m} / \mathrm{z}\left(\mathrm{M}+\mathrm{H}^{+}\right)$Anal. for $\mathrm{C}_{22} \mathrm{H}_{33} \mathrm{ClN}_{4} \mathrm{O}_{6} \mathrm{Na}$ : calcd mass 507.1986. Found 507.2000. Anal. HPLC $\left(100 \% \mathrm{H}_{2} \mathrm{O} / 0.1 \%\right.$ TFA $\rightarrow 100 \% \mathrm{CH}_{3} \mathrm{CN} /$ $0.1 \%$ TFA over $30 \mathrm{~min}$ ).

\section{Methyl 5-(Azepan-1-yl)-3-((tert-butoxycarbonyl)amino)-pyrazine-2-carboxylate (37).}

To a mixture of chloropyrazine 36 (3.30 g, $6.8 \mathrm{mmol}), \mathrm{Pd} / \mathrm{C}$ (476 mg, $10 \mathrm{~mol} \%)$, and $\mathrm{MgO}$ (408 mg, $10.2 \mathrm{mmol}$ ) was added dry $\mathrm{MeOH}(50 \mathrm{~mL})$. The flask was charged with $\mathrm{H}_{2}(1$ atm), and the reaction mixture was stirred at $\mathrm{rt}$ for 2 days. The solvent was evaporated and the residue redissolved in EtOAc and filtered through Celite, eluting with EtOAc. The organic layer was washed with brine, dried $\left(\mathrm{MgSO}_{4}\right)$, and evaporated to give the crude mono Boc-protected product 37 as a white solid (2.22 g, 92\%). Crude product showed sufficient purity and was used in the next step with further purification. Mp $97-100{ }^{\circ} \mathrm{C} .{ }^{1} \mathrm{H}$ NMR (400 $\left.\mathrm{MHz}, \mathrm{CDCl}_{3}\right) \delta 10.28(\mathrm{~s}, 1 \mathrm{H}), 7.68(\mathrm{~s}, 1 \mathrm{H}), 3.94(\mathrm{~s}, 3 \mathrm{H}), 3.62(\mathrm{br} \mathrm{s}, 4 \mathrm{H}), 1.58(\mathrm{br} \mathrm{s}, 4 \mathrm{H})$, 1.53 (s, 9H), 1.84 (br s, $4 \mathrm{H}) .{ }^{13} \mathrm{C} \mathrm{NMR}\left(\mathrm{CDCl}_{3}\right): \delta 167.3,153.8,150.6,150.3,122.4,113.5$, 80.7, 52.3, 47.9, 28.3, 27.4, 26.6. HRESI-MS $m / z\left(\mathrm{M}+\mathrm{H}^{+}\right)$Anal. for $\mathrm{C}_{17} \mathrm{H}_{27} \mathrm{~N}_{4} \mathrm{O}_{4}$ : 351.2032. Found 351.2032. Anal. HPLC $\left(100 \% \mathrm{H}_{2} \mathrm{O} / 0.1 \% \mathrm{TFA} \rightarrow 100 \% \mathrm{CH}_{3} \mathrm{CN} / 0.1 \%\right.$ TFA over $30 \mathrm{~min})$.

\section{Methyl 5-(Azepan-1-yl)-6-bromo-3-((tert-butoxycarbonyl)-amino)pyrazine-2-carboxylate (38).}

To a solution of pyrazine methyl ester $37(2.10 \mathrm{~g}, 6.0 \mathrm{mmol})$ in $\mathrm{CH}_{2} \mathrm{Cl}_{2}(90 \mathrm{~mL})$ was added $\mathrm{N}$-bromosuccinimide $(1.18 \mathrm{~g}, 6.6 \mathrm{mmol})$, and the reaction mixture was stirred at $\mathrm{rt}$ for $5 \mathrm{~h}$. The mixture was treated with water $(50 \mathrm{~mL})$ and stirred for $10 \mathrm{~min}$. The organic layer was separated, washed with sodium bicarbonate solution $(2 \times 50 \mathrm{~mL})$ and brine $(50 \mathrm{~mL})$, dried $\left(\mathrm{MgSO}_{4}\right)$, and concentrated. The residue was purified by silica gel flash column chromatography using EtOAc/pet. spirit (10-15\%) to give $38(1.56 \mathrm{~g}, 60 \%)$ as a white solid. Mp 114-115 ${ }^{\circ} \mathrm{C} .{ }^{1} \mathrm{H}$ NMR $\left(\mathrm{CDCl}_{3}\right): \delta 10.04(\mathrm{~s}, 1 \mathrm{H}), 3.95$ (t, $\left.J=6.0 \mathrm{~Hz}, 4 \mathrm{H}\right), 3.92(\mathrm{~s}, 3 \mathrm{H})$, 1.89 (br s, $4 \mathrm{H}), 1.60(\mathrm{t}, J=3.1 \mathrm{~Hz}, 4 \mathrm{H}), 1.52(\mathrm{~s}, 9 \mathrm{H}) .{ }^{13} \mathrm{C} \mathrm{NMR}\left(\mathrm{CDCl}_{3}\right): \delta 166.3,153.0$, 150.0, 147.7, 114.8, 111.9, 81.0, 52.5, 51.4, 28.2, 28.2, 26.8. MS (ESI): $\mathrm{m} / \mathrm{z} 429(\mathrm{M}+\mathrm{H})^{+}$. HRESI-MS $m / z\left(\mathrm{M}+\mathrm{Na}^{+}\right)$Anal. for $\mathrm{C}_{17} \mathrm{H}_{25} \mathrm{BrN}_{4} \mathrm{O}_{4} \mathrm{Na}$ : calcd mass 451.0957. Found 451.0959. Anal. HPLC $\left(100 \% \mathrm{H}_{2} \mathrm{O} / 0.1 \%\right.$ TFA $\rightarrow 100 \% \mathrm{CH}_{3} \mathrm{CN} / 0.1 \%$ TFA over $\left.30 \mathrm{~min}\right)$. 


\section{Methyl 5-(Azepan-1-yl)-3-((tert-butoxycarbonyl)amino)-6-(pyrimidin-2-ylamino)pyrazine-2- carboxylate (39).}

A resealable Schlenk tube was charged with bromopyrazine 38 (429 mg, $1.0 \mathrm{mmol}), 2$ aminopyrimidine (135 mg, $1.4 \mathrm{mmol}), \mathrm{Pd}_{2}(\mathrm{dba})_{3}(10 \mathrm{mg}, 0.011 \mathrm{mmol}, 1 \mathrm{~mol} \% \mathrm{Pd})$, Xantphos (13 mg, $0.022 \mathrm{mmol}), \mathrm{Cs}_{2} \mathrm{CO}_{3}(426 \mathrm{mg}, 1.4 \mathrm{mmol})$, and degassed dioxane (5 mL). The Schlenk tube was capped and carefully subjected to three cycles of evacuation -backfilling with $\mathrm{N}_{2}$. It was then sealed and the reaction mixture heated at $100{ }^{\circ} \mathrm{C}$ for $20 \mathrm{~h}$. The mixture was cooled to rt, diluted with THF $(20 \mathrm{~mL})$, filtered through Celite, and concentrated. The residue was purified by silica gel flash column chromatography using EtOAc/pet. spirit (50-60\%) to give $39(130 \mathrm{mg}, 30 \%)$ as a white solid. Mp $168-170{ }^{\circ} \mathrm{C} .{ }^{1} \mathrm{H}$ $\operatorname{NMR}\left(\mathrm{CDCl}_{3}\right): \delta 10.16(\mathrm{~s}, 1 \mathrm{H}), 8.35(\mathrm{~d}, J=4.8 \mathrm{~Hz}, 2 \mathrm{H}), 7.23(\mathrm{~s}, 1 \mathrm{H}), 6.71(\mathrm{t}, J=4.8 \mathrm{~Hz}$, $1 \mathrm{H}), 3.90(\mathrm{~s}, 3 \mathrm{H}), 3.77$ (t, $J=6.0 \mathrm{~Hz}, 4 \mathrm{H}), 1.73$ (br s, $4 \mathrm{H}), 1.53(\mathrm{~s}, 9 \mathrm{H}), 1.48(\mathrm{br} \mathrm{s}, 4 \mathrm{H}) .{ }^{13} \mathrm{C}$ NMR $\left(\mathrm{CDCl}_{3}\right): \delta 166.8,161.3,158.3,152.3,150.2,147.9,126.6,113.2,111.5,80.5,52.4$, 50.5, 28.3, 28.0, 26.8. MS (ESI): $m / z 444(\mathrm{M}+\mathrm{H})^{+}$. HRESI-MS $m / z\left(\mathrm{M}+\mathrm{H}^{+}\right)$Anal. for $\mathrm{C}_{21} \mathrm{H}_{30} \mathrm{~N}_{7} \mathrm{O}_{4}$ : calcd mass 444.2359. Found 444.2374. Anal. HPLC $\left(100 \% \mathrm{H}_{2} \mathrm{O} / 0.1 \%\right.$ TFA $\rightarrow 100 \% \mathrm{CH}_{3} \mathrm{CN} / 0.1 \%$ TFA over $30 \mathrm{~min}$ ).

\section{Methyl 3-Amino-5-(azepan-1-yl)-6-(pyrimidin-2-ylamino)-pyrazine-2-carboxylate (40).}

To a solution of Boc-protected pyrazine $39(60 \mathrm{mg}, 0.135 \mathrm{mmol})$ in $\mathrm{CH}_{2} \mathrm{Cl}_{2}(4 \mathrm{~mL})$ at $0{ }^{\circ} \mathrm{C}$ was added TFA $(0.1 \mathrm{~mL})$, and the mixture was stirred at room temperature overnight. The mixture was concentrated in vacuo and the residue partitioned between $\mathrm{CH}_{2} \mathrm{Cl}_{2}(20 \mathrm{~mL})$ and saturated $\mathrm{NaHCO}_{3}$ solution $(20 \mathrm{~mL})$. The organic layer was dried $\left(\mathrm{MgSO}_{4}\right)$ and concentrated and the residue purified by silica gel flash column chromatography using EtOAc/pet. spirit (60-70\%) to give $40(45 \mathrm{mg}, 98 \%)$ as a gray solid. Mp $194-196{ }^{\circ} \mathrm{C} .{ }^{1} \mathrm{H}$ NMR $\left(\mathrm{CDCl}_{3}\right): \delta 8.34(\mathrm{~d}, J=4.8 \mathrm{~Hz}, 2 \mathrm{H}), 7.04(\mathrm{~s}, 1 \mathrm{H}), 6.67(\mathrm{t}, J=4.8 \mathrm{~Hz}, 1 \mathrm{H}), 6.20$ (br s, $2 \mathrm{H}, \mathrm{NH}_{2}$ ), 3.87 (s, $\left.3 \mathrm{H}\right), 3.67$ (t, $\left.J=6.0 \mathrm{~Hz}, 4 \mathrm{H}\right), 1.69$ (br s, $\left.4 \mathrm{H}\right), 1.48$ (t, $\left.J=3.2 \mathrm{~Hz}, 4 \mathrm{H}\right) .{ }^{13} \mathrm{C}$ $\operatorname{NMR}\left(\mathrm{CDCl}_{3}\right): \delta 166.8,161.8,158.4,154.2,153.5,124.3,112.8,109.4,52.0,50.6,50.3$, 28.2, 26.7. HRESI-MS $m / z\left(\mathrm{M}+\mathrm{H}^{+}\right)$Anal. for $\mathrm{C}_{16} \mathrm{H}_{22} \mathrm{~N}_{7} \mathrm{O}_{2}$ : calcd mass 344.1835. Found 344.1848. Anal. HPLC $\left(100 \% \mathrm{H}_{2} \mathrm{O} / 0.1 \%\right.$ TFA $\rightarrow 100 \% \mathrm{CH}_{3} \mathrm{CN} / 0.1 \%$ TFA over $\left.30 \mathrm{~min}\right)$.

\section{3-Amino-5-(azepan-1-yl)-N-carbamimidoyl-6-(pyrimidin-2-ylamino)pyrazine-2-carboxamide (41). \\ General method D using $40(35 \mathrm{mg}, 0.1 \mathrm{mmol})$ gave $41(30 \mathrm{mg}, 81 \%)$ as a yellow solid. Mp 210-212 ${ }^{\circ} \mathrm{C} .{ }^{1} \mathrm{H}$ NMR ( $\left.\mathrm{CD}_{3} \mathrm{OD}\right): \delta 8.25(\mathrm{~d}, J=4.9 \mathrm{~Hz}, 2 \mathrm{H}), 6.70(\mathrm{t}, J=4.9 \mathrm{~Hz}, 1 \mathrm{H}), 3.70(\mathrm{t}$, $J=6.0 \mathrm{~Hz}, 4 \mathrm{H}), 1.63(\mathrm{br} \mathrm{s}, 4 \mathrm{H}), 1.43(\mathrm{t}, J=3.1 \mathrm{~Hz}, 4 \mathrm{H}) .{ }^{13} \mathrm{C}$ NMR $\left(\mathrm{CD}_{3} \mathrm{OD}\right): \delta 165.7$, 162.2, 158.3, 155.6, 154.9, 154.8, 124.1, 112.3, 108.0, 50.4, 27.8, 26.2. MS (ESI): $\mathrm{m} / \mathrm{z} 371$ $(\mathrm{M}+\mathrm{H})^{+}$. HRESI-MS $m / z\left(\mathrm{M}+\mathrm{H}^{+}\right)$Anal. for $\mathrm{C}_{16} \mathrm{H}_{23} \mathrm{~N}_{10} \mathrm{O}$ : calcd mass 371.2056. Found 371.2057. Anal. HPLC $\left(100 \% \mathrm{H}_{2} \mathrm{O} / 0.1 \% \mathrm{TFA} \rightarrow 100 \% \mathrm{CH}_{3} \mathrm{CN} / 0.1 \%\right.$ TFA over $30 \mathrm{~min}, t_{\mathrm{R}}$ $=14.3 \mathrm{~min}$ ).}

\section{Cell Culture Conditions.}

ATCC certified cell lines were used for all experiments. Cells were cultured at $37{ }^{\circ} \mathrm{C}, 95 \%$ humidity, and 5\% (v/v) $\mathrm{CO}_{2}$ in a ThermoScientific Heracell $150 \mathrm{i} \mathrm{CO}_{2}$ incubator (ThermoScientific) and maintained in DMEM/Hi glucose culture medium supplemented 
with $10 \%$ heat-inactivated FCS. Adherent cells were passaged via the addition of trypsin/ $0.05 \%$ EDTA solution (GIBCO), followed by centrifugation and resuspension in fresh media using a $5 \mathrm{~mL}$ Luerlock syringe fitted with an 18-gauge needle (Terumo, Tokyo, Japan). All cell lines were routinely tested for the presence of mycoplasma infection.

\section{Fluorometric uPA Activity Assay.}

Urokinase from human kidney cells (catalog no. U4010, Sigma-Aldrich, St. Louis, MO, USA) was diluted to $900 \mathrm{nM}$, aliquoted, and stored at $-80{ }^{\circ} \mathrm{C}$ until use in experiments (typically 4-5 plates assayed on the same day). Thawed enzyme stocks were maintained at $20^{\circ} \mathrm{C}$ between during assays using a Nalgene Labtop Cooler Jr (catalog no. 5115-0012, Thermo-Fisher Scientific, Waltham, MA, USA). A $5 \mathrm{mM}$ stock solution of urokinase fluorescent substrate III (Z-Gly-Gly-Arg-AMC, Calbiochem catalog no. 672159, Merck Millipore, MA, USA, human uPA $K_{\mathrm{m}}=400 \mu \mathrm{M}$ ) was prepared in $\mathrm{dH}_{2} \mathrm{O} / 10 \%$ DMSO and aliquoted with storage at $-20^{\circ} \mathrm{C}$. Assay buffer (20 mM HEPES, pH 7.4, $100 \mathrm{mM} \mathrm{NaCl}, 0.5$ mM EDTA, $0.01 \% \mathrm{v} / \mathrm{v}$ Tween-20) was prepared and used within 7 days with storage at $4{ }^{\circ} \mathrm{C}$. On the day of assay, compound stocks (20-50 mM in anhydrous DMSO) were serially diluted in assay buffer in a clear Greiner CELLSTAR 96-well plate on ice (Greiner Bio-One $\mathrm{GmBH}$, Kremsmünster, Austria) to give each dilution at $2 \times$ the final assayed concentration. In a separate black Greiner CELLSTAR 96-well plate (Greiner Bio-One GmBH, Kremsmünster, Austria), residual volumes of assay buffer were added via multichannel pipet to compound fluorescence blanks and inhibited enzyme control wells, as appropriate. Next, $90 \mu \mathrm{L}$ of dilute fluor ( $250 \mu \mathrm{M}$ final) was added to all wells, followed by $100 \mu \mathrm{L}$ of $2 \times$ compound dilution to appropriate wells. Immediately prior to assay, $900 \mathrm{nM}$ uPA stock was diluted to $15 \mathrm{nM}$ and $10 \mu \mathrm{L}$ of this dilution was added to all relevant wells, initiating the reaction. Reaction progress was monitored using a POLARStar OMEGA fluorescence plate reader (fluorescence intensity mode, top-optic, ex $355 \mathrm{~nm}$, em $450 \mathrm{~nm}, 20$ flashes per well, double orbital shaking for $3 \mathrm{~s}$ prior to every read, cycle time $60 \mathrm{~s}, 45-60$ cycles, incubation at $37^{\circ} \mathrm{C}$, BMG-Labtech, Ortenberg, Germany). Changes in fluorescence over a $15 \mathrm{~min}$ period from the linear portion of the reaction progress curve were used to determine $\mathrm{IC}_{50}$ values by fitting log-transformed data to a sigmoidal dose-response curve in GraphPad Prism v7.0 (GraphPad Software, La Jolla, CA, USA). Experiments with Mouse uPA (active mouse urokinase, HMW, Molecular Innovations Inc., MI, USA) were performed as above with a final enzyme concentration of $20 \mathrm{nM}\left(\mathrm{K}_{\mathrm{m}}=580 \mu \mathrm{M}\right) . \mathrm{K}_{\mathrm{i}}$ values were calculated according to the methods of Cheng and Prussof. ${ }^{52}$

\section{Chromogenic Trypsin-like Serine Protease (TLSP) Activity Assays.}

For experiments using uPA (catalog no. U4010, Sigma-Aldrich, St. Louis, MO, USA), trypsin, $\mathrm{tPA}$, thrombin, plasmin, and factor $\mathrm{Xa}$, concentrated stocks were prepared in deionized $\mathrm{H}_{2} \mathrm{O}$ and stored at $-80{ }^{\circ} \mathrm{C}$ until thawed for use. Thawed enzyme stocks were maintained at $-20^{\circ} \mathrm{C}$ between assays using a Nalgene Labtop Cooler Jr (catalog no. 51150012, Thermo-Fisher Scientific, Waltham, MA, USA). A $312.5 \mu \mathrm{M}$ stock solution of S-2288 chromogenic substrate (HD-Ile-L-Pro-L-Arg-p-nitoraniline, Chromogenix, MA, USA, uPA $K_{\mathrm{m}}=200 \mu \mathrm{M}$, trypsin $K_{\mathrm{m}}=28 \mu \mathrm{M}$, tPA $K_{\mathrm{m}}=300 \mu \mathrm{M}$, thrombin $K_{\mathrm{m}}=3 \mu \mathrm{M}$, plasmin $K_{\mathrm{m}}=$ $9 \mathrm{mM}$ ) was prepared in deionized $\mathrm{H}_{2} \mathrm{O}$ and aliquoted with storage at $-20{ }^{\circ} \mathrm{C}$. Assay buffer (10 mM HEPES, $150 \mathrm{mM} \mathrm{NaCl}, 0.01 \%$ v/v Tween-20, pH 7.4) was prepared and used 
within 7 days with storage at $4{ }^{\circ} \mathrm{C}$. On the day of assay, compound stocks $(20-50 \mathrm{mM}$ in DMSO) were serially diluted in assay buffer in a clear Greiner CELLSTAR 96-well plate on ice (Greiner Bio-One GmBH, Kremsmünster, Austria) to give each dilution at 10× the final assay concentration. In a separate Greiner CELLSTAR 96-well plate (Greiner Bio-One $\mathrm{GmBH}, \mathrm{Kremsmünster,} \mathrm{Austria)} \mathrm{residual} \mathrm{volumes} \mathrm{of} \mathrm{assay} \mathrm{buffer} \mathrm{were} \mathrm{added} \mathrm{via}$ multichannel pipet to compound fluorescence blanks and inhibited enzyme control wells, as appropriate. Next, $80 \mu \mathrm{L}$ of dilute substrate ( $250 \mu \mathrm{M}$ final) was added to all wells, followed by $10 \mu \mathrm{L}$ of $10 \times$ compound dilution to appropriate wells. Immediately prior to assay, concentrated enzyme stocks were diluted to $100 \mathrm{nM}$ and $10 \mu \mathrm{L}$ of this dilution was added to all relevant wells, initiating the reaction (final enzyme concentration $=10 \mathrm{nM}$ ). DMSO was present at a final concentration of $1 \% \mathrm{v} / \mathrm{v}$. Change in absorbance over time at $405 \mathrm{~nm}$ was measured at $37{ }^{\circ} \mathrm{C}$ using a Molecular Devices SpectraMax Plus 384-well plate reader (Molecular Devices LLC, Sunnyvale, CA, USA). $\mathrm{IC}_{50}$ values were determined by plotting percentage of residual activity (V0) versus log drug concentration and fitted to sigmoidal dose response curves using GraphPad Prism v7.0 (GraphPad Software, La Jolla, CA, USA). The following chromogenic substrates and final enzyme concentrations were used for experiments with other TLSPs. S-2366, L-pyroGlu-L-Pro-L-Arg-p-nitroaniline, Chromogenix, MA, USA, [human plasma kallikrein] $=23.5 \mathrm{nM}, K_{\mathrm{m}}=430 \mu \mathrm{M}$, [factor XIa] $=2 \mathrm{nM}, K_{\mathrm{m}}=400 \mu \mathrm{M}$, [activated protein C] $=10 \mathrm{nM}, K_{\mathrm{m}}=200 \mu \mathrm{M}$.

\section{Crystallization and X-ray Data Collection.}

Expression and purification of recombinant human uPA protease domain were performed as previously described. ${ }^{78}$ Crystals were grown by the sitting drop vapor diffusion method and were obtained by equilibrating against a reservoir solution containing $50 \mathrm{mM}$ sodium citrate (pH 4.6) and 2.0 M ammonium sulfate supplemented with 5\% PEG400 at room temperature for a period of 3 days. For uPA:inhibitor cocomplexes at $\mathrm{pH} 7.4$, the crystals were soaked for 1 week in new soaking buffer (40\% PEG4000, 100 mM Tris-HCl, pH 7.4) containing 100 $\mu \mathrm{M}$ compound. Prior to $\mathrm{X}$-ray data collection, crystals were soaked in a cryoprotectant solution containing $20 \%$ glycerol and snap-frozen in liquid $\mathrm{N}_{2}$. X-ray diffraction data of the crystals were collected at the BL17U beamline, Shanghai Synchrotron Radiation Facility (SSRF). The diffraction data were indexed and integrated using the HKL2000 program package. ${ }^{79}$ Structures were solved by molecular replacement ${ }^{80}$ using the uPA structure (PDB code 4DVA) ${ }^{81}$ as the search model. The electron density for the compound was clearly visible in the uPA active sites and was modeled based on the $F_{\mathrm{O}}-F_{\mathrm{c}}$ difference map. The structures were refined using the CCP4 program package ${ }^{79}$ and manually adjusted using the molecular graphics program COOT $^{82}$ iteratively until convergence. Solvent molecules were added using a $F_{\mathrm{O}}-F_{\mathrm{c}}$ Fourier difference map at $2.5 \sigma$ in the final refinement step. Statistics for data collection and final model refinement are summarized in the Supporting Information Table S1.

\section{EnPlex Assays.}

Enplex superfamily wide serine hydrolase activity screening was performed as described in ref 54 . 


\section{Cytotoxicity Assays.}

Subconfluent cells ( $~ 70-80 \%$ confluence) were harvested and dissociated into a single cell suspension. Cells were seeded at a density of 5000 cells/well (final volume $90 \mu \mathrm{L}$ ) into a Greiner CELLSTAR 96-well plate (Greiner Bio-One GmBH, Kremsmünster, Austria) via multichannel pipet and incubated for $18 \mathrm{~h}$ prior to the addition of compounds. Compounds were serially diluted from 20 to $50 \mathrm{mM}$ DMSO stocks in culture media in a separate 96-well plate under sterile conditions to give $10 \times$ stocks $(10 \mathrm{nM}$ to $1 \mathrm{mM})$ on the day of compound addition. $10 \mu \mathrm{L}$ of the $10 \times$ compound/media stocks or matched vehicle/media solutions (for vehicle controls; final vehicle concentration was equal to $0.2 \%$ ) were transferred via multichannel pipet into the assay plate containing $90 \mu \mathrm{L}$ of cell suspension via multichannel pipet to give the treated assay plate at the final $1 \times$ drug concentrations $(1 \mathrm{nM}$ to $100 \mu \mathrm{M}, n=$ 4 wells at each concentration). Drug blanks or vehicle media blanks were included as appropriate at each concentration to correct for intrinsic color of the compounds and the phenol red containing media. $200 \mu \mathrm{L}$ media was added to outside wells to decrease evaporation but was not used in the assay itself. Compound-treated plates were incubated for $48 \mathrm{~h}$ prior to development. After $48 \mathrm{~h}$, plates were removed from the incubator and $20 \mu \mathrm{L}$ of CellTiter 96 Aqueous One solution cell proliferation assay (catalog no. G3581, Promega Corporation, Fitchburg, WI, USA) was added to each well. Plates were incubated for a further $2 \mathrm{~h}$ prior to reading at $490 \mathrm{~nm}$ using a SpectraMax Plus 384-well plate reader (Molecular Devices LLC, Sunnyvale, CA, USA). $\mathrm{IC}_{50}$ values (defined as the concentration of each treatment causing 50\% inhibition of maximal cell viability) were determined using a logarithmic sigmoidal dose-response curve using the variable slope parameter to determine $\mathrm{IC}_{50}$ using GraphPad Prism v7.0 (GraphPad Software, La Jolla, CA, USA).

\section{ENaC Inhibition Assays.}

HEK-293 cells were cultured in Dulbecco's modified Eagle medium/nutrient mixture F-12 (D-MEM/F-12) supplemented with $10 \%$ fetal bovine serum, $100 \mathrm{U} / \mathrm{mL}$ penicillin $\mathrm{G}$ sodium, $100 \mu \mathrm{g} / \mathrm{mL}$ streptomycin sulfate, and appropriate selection antibiotics. Semiconfluent cells were transfected with cDNA for $\mathrm{ENaC} a, \beta$, and $\gamma$ subunits (genes SCNN1A, SCNN1B, and SCNN1G) prior to experimentation. Cells were plated at a density of 20000 cells/well in a 384-well black wall, flat clear bottom microtiter plates (type: BD Biocoat poly-D-lysine multiwell cell culture plate) and incubated at $37{ }^{\circ} \mathrm{C}$ overnight. Following manufacturer instructions, cells were loaded with $20 \mu \mathrm{L}$ of membrane potential dye solution for $30 \mathrm{~min}$ at $37{ }^{\circ} \mathrm{C}$ from the FLIPR membrane potential red dye fluorescence assay kit (Molecular Devices, Sunnyvale, CA, USA). This assay uses fluorometric detection of changes in membrane potential following modulation of transmembrane ion channel/exchanger activity, i.e., antagonism of stimulated $\mathrm{ENaC}$ activity in this case. Drug, vehicle, and control solutions were prepared from frozen stocks in DMSO on the day of experimentation through dilution into HEPES-buffered physiological salt solution ( $\mathrm{NaCl} 137 \mathrm{mM}, \mathrm{KCl} 4 \mathrm{mM}, \mathrm{CaCl}_{2}$ $1.8 \mathrm{mM}, \mathrm{MgCl}_{2} 1 \mathrm{mM}$, HEPES $10 \mathrm{mM}$, glucose $10 \mathrm{mM}$, DMSO 0.3\% v/v, pH 7.4) in a 384well microtiter plate ready for addition to the assay plate by the FLIPR ${ }^{\text {TETRA }}$ instrument. All analogs were tested at 8 concentrations ( $n=4$ wells at each concentration). Positive control cells were stimulated with $1 \mu \mathrm{M}$ S-3969 (ENaC agonist, $n=4$ wells) ${ }^{83}$ Negative control cells were first stimulated with $1 \mu \mathrm{M} \mathrm{S}$-3969 and then treated with $30 \mu \mathrm{M}$ benzamil (potent $\mathrm{ENaC}$ antagonist, $n=4$ wells). Following dye loading, cells were preincubated with 
$5 \mu \mathrm{L}$ of drug, vehicle, or control solutions for $5 \mathrm{~min}$. Next $5 \mu \mathrm{L}$ of S-3939 solution (final $[\mathrm{S}-3969]=1 \mu \mathrm{M}$ ) was added to stimulate ENaC activity. All data were normalized to agonist-treated positive and antagonist-treated negative controls such that the agonist-treated signal corresponded to $100 \%$ activity and antagonist-treated signal corresponded to $0 \%$ activity. Normalized data were used to determine $\mathrm{IC}_{50}$ values for each analog using the $\log$ [inhibitor]-normalized response variable slope algorithm in GraphPad Prism v7.0 (GraphPad Software, La Jolla, CA, USA). These experiments were performed under contract by Charles River Laboratories, Cleveland, OH, USA.

\section{Rat Diuresis and Kaliuresis Model.}

Sixty-four male 21 week old Sprague-Dawley rats were randomized into 8 groups of 8 animals. All test animals received one intraperitoneal (ip) injection of either vehicle or 25 $\mathrm{mg} / \mathrm{kg}$ acetazolamide followed by one intravenous (iv) injection of vehicle, $1.5 \mathrm{mg} / \mathrm{kg}$ amiloride or $1.5 \mathrm{mg} / \mathrm{kg}$ amiloride derivative. Acetazolamide was administered at an injection volume of $1 \mathrm{~mL} / \mathrm{kg}$ and concentration of $25 \mathrm{mg} / \mathrm{mL}$. Amiloride, HMA, 18, and 26 were administered at an injection volume of $1 \mathrm{~mL} / \mathrm{kg}$ and concentration of $1.5 \mathrm{mg} / \mathrm{mL}$. Monosodium acetazolamide was formulated in $0.9 \%$ saline. Amiloride and HMA were formulated in $50 \mathrm{mM}$ acetate buffer $(\mathrm{pH} 5.5) / 5 \% \mathrm{v} / \mathrm{v}$ DMSO, and 18 and 26 were formulated in $50 \mathrm{mM}$ acetate buffer (pH 5.5)/5\% v/v DMSO/0.5\% v/v Tween-80. Immediately following dosing, all animals were placed into metabolic cages and total urine was collected over $6 \mathrm{~h}$. A control group receiving ip and iv administrations of vehicle was also included for comparison. Urine $\mathrm{Na}^{+}$and $\mathrm{K}^{+}$levels were quantitated using a Konelab 20 XTi biochemistry analyzer $(\mathrm{LLQ}=20 \mathrm{mM})$. This experiment was approved by the Austin Health Animal Ethics Committee (A2016/05420) and was conducted in accordance with the Australian Code of Practice for the Care and Use of Animals for Scientific Purposes, seventh edition, 2004. Statistical analyses were performed using a one-way ANOVA with Dunnett's post hoc test in GraphPad Prism v7.0 (GraphPad Software, La Jolla, CA, USA).

\section{Mouse Lung Metastasis Model.}

All animal studies were approved by the Institutional Animal Care and Use Committee at the University of Wollongong (AE1708). Male and female NOD.Cg-Prkdc<scid >IL2 $\mathrm{rg}<\mathrm{tm} 1 \mathrm{Wj} 1>$ SzJAusb mice (Australian BioResources, Moss Vale, NSW, Australia) at 7-8 weeks of age were randomized into treatment groups ( $n=4-6 /$ treatment group). Treatments commenced 1 day prior to tail vein administration of $2.5 \times 10^{5}$ luciferase-tagged HT-1080 human fibrosarcoma cells (BioWare Brite HT-1080-Red-Fluc) in an injection volume of $100 \mu \mathrm{L}$ of PBS [except sham control $(n=1)$ ]. Daily ip injections continued to day 20 with $7.5 \mathrm{mg} / \mathrm{kg}$ amiloride, 18,26 , or vehicle $(50 \mathrm{mM}$ acetate buffer, $\mathrm{pH} 5.5,7.5 \% \mathrm{v} / \mathrm{v}$ DMSO, $0.75 \%$ Tween 80 ). Animals were weighed and assessed for clinical signs daily. Humane end points of $>15 \%$ acute weight loss (from previous 7 day maximum individual weight) or $>20 \%$ chronic weight loss (from all-time individual maximum weight) or a body condition score $>3$ was employed. On day 21 , all remaining animals were euthanized via slow $\mathrm{CO}_{2}$ asphyxiation, weighed, necropsied, and had their lungs removed. Lungs were snap frozen in liquid $\mathrm{N}_{2}$ and stored at $-80{ }^{\circ} \mathrm{C}$ until homogenization. Lungs were homogenized on ice using a PRO Scientific Bio-Gen PRO200 homogenizer in cell lysis buffer $(150 \mathrm{mM}$ $\mathrm{NaCl}, 10 \mathrm{mM}$ Tris base, $1 \%$ Triton $\mathrm{X} 100 \mathrm{v} / \mathrm{v}, \mathrm{pH} 8.0$ ) and centrifuged twice at $4{ }^{\circ} \mathrm{C}$ and 
$1000 \mathrm{rpm}$ for $15 \mathrm{~min}$ to remove cell debris. Homogenates were prepared to approximately $50 \mathrm{mg} / \mathrm{mL}$ protein based on individual wet lung weights. Luciferase activity was quantified according to the manufacturer's instructions using the Molecular Diagnostics SpectraMax Glo Steady-Luc report assay kit. Luciferase assays were conducted in clear-bottomed white walled 96-well plates (Nunc) using a BMG Labtech PolarSTAR multimode plate reader.

Data were analyzed using GraphPad Prism v7.0, and statistical significance was determined using the one-way ANOVA with Dunnett's post hoc test.

\section{Orthotopic Model of Pancreatic Cancer.}

The orthotopic model has been described in detail previously. ${ }^{67,84}$ In brief, 6- to 8-week-old female athymic nude mice (BALB/c nu/nu) were anesthetized and an incision was made in the left flank, followed by exteriorization of the spleen and tail of the pancreas. A mixture of human PC cells (AsPC-1) $1 \times 10^{6}+$ human PSCs (hPSCs) $1 \times 10^{6}$ in $50 \mu \mathrm{L}$ of PBS was injected into the tail of the pancreas. AsPC-1 cells were chosen for their high implantation rate (100\% in our hands) and high reproducibility of tumor growth rates and metastasis. After intrapancreatic injection, organs were reinternalized and the abdomen was surgically closed. Mice receiving treatment were randomized into subgroups ( $n=4$ mice per group) on postsurgical day 7 . These mice were treated as follows for 4 weeks: gemcitabine $(75 \mathrm{mg} / \mathrm{kg}$ BIW oral gavage); 18 or 26 (10 mg/kg/day ip or vehicle (daily ip, BIW oral gavage). Compounds were formulated for ip injection in $50 \mathrm{mM}$ sodium acetate buffer (pH 5.5)/ 10\% (v/v) DMSO/1\% (w/v/) Kolliphor HS-15 and filtered through $0.22 \mu \mathrm{m}$ PVDF syringe-driven filters under sterile conditions. Pancreatic tumor growth was monitored by palpation. Mice were killed at 4 weeks after first ip injection. Tumors were resected and assessed for size and volume by two separate observers (tumor volume was calculated to two decimal points, according to an established formula $(1 / 2$ (length $\times$ breadth $\times$ width $)$ using digital Vernier calipers (Intech Tools, Thomas Town, VIC, Australia). ${ }^{84}$ The abdominal cavity, mesentery, spleen, liver, and lungs were examined and scored according to the presence or absence of visible macrometastatic nodules. These organs were then excised and fixed in $10 \%$ neutral buffered formalin overnight and processed using the Leica Peloris dual retort tissue processor (Germany). Histological staining was performed on $4 \mu \mathrm{m}$ sections deparaffinised in xylene and rehydrated using graded ethanol washes. Haematoxylin and eosin staining were performed on a Leica autostainer XL.

This experiment was approved by the University of New South Wales Animal Care and Ethics Committee (Approval Number 15/106A) and was conducted in accordance with ARRIVE Guidelines.

\section{Supplementary Material}

Refer to Web version on PubMed Central for supplementary material.

\section{ACKNOWLEDGMENTS}

This work was funded by the Australian National Health and Medical Research Council (NHMRC, Project Grant APP1100432). The authors gratefully acknowledge Dr. Arnault Massink, Mrs Corine van Veen and Prof Ad P. Ijzerman, Leiden Academic Centre for Drug Research, Leiden University, for $\mathrm{A}_{2 \mathrm{~A}}$ activity screening. D.C.J. 
acknowledges funding from an NIH T32 GM115327-Tan training grant. D.A.B and D.C.J. acknowledge funding from a MSKCC Core Grant P30 (Grant CA008748).

\section{ABBREVIATIONS USED}

\begin{tabular}{|c|c|}
\hline ABPP & activity-based protein profiling \\
\hline ASIC1a & acid-sensing ion channel 1a \\
\hline C1r & complement $\mathrm{C} 1 \mathrm{r}$ subcomponent \\
\hline C1s & complement component $1 \mathrm{~s}$ \\
\hline DDHD1 & phosphatidic acid-preferring phospholipase A1 \\
\hline DIPEA & diisopropylethylamine \\
\hline DOCA & deoxycorticosterone acetate \\
\hline DPP7 & dipeptidyl peptidase 2 \\
\hline ENaC & epithelial sodium channel \\
\hline FLT3-ITD+ & Fms-like tyrosine kinase internal tandem duplication positive \\
\hline GABA-A & $\gamma$-aminobutyric acid $_{\mathrm{A}}$ receptor \\
\hline Gem & gemcitabine \\
\hline GIRK & G-protein-coupled inwardly rectifying potassium channel \\
\hline GnRHR & gonadotropin-releasing hormone receptor \\
\hline GPCR & G-protein-coupled receptor \\
\hline $\mathrm{hA}_{2 \mathrm{~A}} \mathrm{AR}-\mathrm{WT}$ & human adenosine $\mathrm{A}_{2 \mathrm{~A}}$ receptor \\
\hline HER2 & human epidermal growth factor receptor 2 \\
\hline HIV-1 & human immunodeficiency virus 1 \\
\hline HMA & 5-(N,N-hexamethylene)amiloride \\
\hline Hpsc & human pancreatic stellate cells \\
\hline KLKb1 & plasma kallikrein \\
\hline LACTB & serine $\beta$-lactamase-like protein \\
\hline NECA & $5^{\prime}$-(N-ethylcarboxamido)adenosine \\
\hline NHE1 & sodium hydrogen exchanger isoform 1 \\
\hline NSB & non-specific binding \\
\hline $\mathbf{P}^{2} \mathbf{X}_{7}$ & $\mathrm{P} 2 \mathrm{X}$ purinoreceptor \\
\hline
\end{tabular}




$\begin{array}{ll}\text { PRCP } & \text { prolylcar-boxypeptidase } \\ \text { SAR } & \text { structure-activity relationship } \\ \text { SOSA } & \text { selective optimization of side activity } \\ \text { TB } & \text { total binding } \\ \text { TLSP } & \text { trypsin-like serine protease } \\ \text { TMPRSS11d } & \text { trypsin and transmembrane protease serine 11D } \\ \text { UPA } & \text { urokinase plasminogen activator } \\ \text { uPAR } & \text { urokinase plasminogen activator receptor }\end{array}$

\section{REFERENCES}

(1). Ulisse S; Baldini E; Sorrenti S; D'Armiento M The urokinase plasminogen activator system: a target for anti-cancer therapy. Curr. Cancer Drug Targets 2009, 9 (1), 32-71. [PubMed: 19200050]

(2). Brungs D; Chen J; Aghmesheh M; Vine KL; Becker TM; Carolan MG; Ranson M The urokinase plasminogen activation system in gastroesophageal cancer: A systematic review and metaanalysis. Oncotarget 2017, 8 (14), 23099-23109. [PubMed: 28416743]

(3). Croucher DR; Saunders DN; Lobov S; Ranson M Revisiting the biological roles of PAI2 (SERPINB2) in cancer. Nat. Rev. Cancer 2008, 8 (7), 535-545. [PubMed: 18548086]

(4). Harris NLE; Vennin C; Conway JRW; Vine KL; Pinese M; Cowley MJ; Shearer RF; Lucas MC; Herrmann D; Allam AH; Pajic M; Morton JP; Australian Pancreatic Cancer Genome Initiative; Biankin AV; Ranson M; Timpson P; Saunders DN SerpinB2 regulates stromal remodelling and local invasion in pancreatic cancer. Oncogene 2017, 36 (30), 4288-4298. [PubMed: 28346421]

(5). Yang JL; Seetoo D; Wang Y; Ranson M; Berney CR; Ham JM; Russell PJ; Crowe PJ Urokinasetype plasminogen activator and its receptor in colorectal cancer: independent prognostic factors of metastasis and cancer-specific survival and potential therapeutic targets. Int. J. Cancer 2000, 89 (5), 431-439. [PubMed: 11008205]

(6). Harris L; Fritsche H; Mennel R; Norton L; Ravdin P; Taube S; Somerfield MR; Hayes DF; Bast RC, Jr. American society of clinical oncology 2007 update of recommendations for the use of tumor markers in breast cancer. J. Clin. Oncol 2007, 25 (33), 5287-5312. [PubMed: 17954709]

(7). Look MP; van Putten WL; Duffy MJ; Harbeck N; Christensen IJ; Thomssen C; Kates R; Spyratos F; Ferno M; Eppenberger-Castori S; Sweep CG; Ulm K; Peyrat JP; Martin PM; Magdelenat H; Brunner N; Duggan C; Lisboa BW; Bendahl PO; Quillien V; Daver A; Ricolleau G; Meijer-van Gelder ME; Manders P; Fiets WE; Blankenstein MA; Broet P; Romain S; Daxenbichler G; Windbichler G; Cufer T; Borstnar S; Kueng W; Beex LV; Klijn JG; O’Higgins N; Eppenberger U; Janicke F; Schmitt M; Foekens JA Pooled analysis of prognostic impact of urokinase-type plasminogen activator and its inhibitor PAI-1 in 8377 breast cancer patients. J. Natl. Cancer. Inst 2002, 94 (2), 116-128. [PubMed: 11792750]

(8). Foekens JA; Peters HA; Look MP; Portengen H; Schmitt M; Kramer MD; Brunner N; Janicke F; Meijer-van Gelder ME; Henzen-Logmans SC; van Putten WL; Klijn JG The urokinase system of plasminogen activation and prognosis in 2780 breast cancer patients. Cancer. Res 2000, 60 (3), 636-643. [PubMed: 10676647]

(9). Duffy MJ; O’Grady P; Devaney D; O’Siorain L; Fennelly JJ; Lijnen HJ Urokinase-plasminogen activator, a marker for aggressive breast carcinomas. Preliminary report. Cancer 1988, 62 (3), 531-533. [PubMed: 3134120]

(10). Estreicher A; Muhlhauser J; Carpentier JL; Orci L; Vassalli JD The receptor for urokinase type plasminogen activator polarizes expression of the protease to the leading edge of migrating 
monocytes and promotes degradation of enzyme inhibitor complexes. J. Cell Biol 1990, 111 (2), 783-792. [PubMed: 2166055]

(11). Lund IK; Illemann M; Thurison T; Christensen IJ; Hoyer-Hansen G uPAR as anti-cancer target: evaluation of biomarker potential, histological localization, and antibody-based therapy. Curr. Drug Targets 2011, 12 (12), 1744-1760. [PubMed: 21707477]

(12). Dohn LH; Illemann M; Høyer-Hansen G; Christensen IJ; Hostmark J; Litlekalsoy J; von der Maase H; Pappot H; Laerum OD Urokinase-type plasminogen activator receptor (uPAR) expression is associated with T-stage and survival in urothelial carcinoma of the bladder. Urol. Oncol 2015, 33 (4), 165.e15-165.e24.

(13). Ploug M; Ellis V Structure-function relationships in the receptor for urokinase-type plasminogen activator. Comparison to other members of the Ly- 6 family and snake venom alpha-neurotoxins. FEBS Lett. 1994, 349 (2), 163-168. [PubMed: 8050560]

(14). Duffy MJ; Duggan C The urokinase plasminogen activator system: a rich source of tumour markers for the individualised management of patients with cancer. Clin. Biochem 2004, 37 (7), 541-548. [PubMed: 15234235]

(15). Rowe RG; Weiss SJ Navigating ECM barriers at the invasive front: the cancer cell-stroma interface. Annu. Rev. Cell Dev. Biol 2009, 25, 567-595. [PubMed: 19575644]

(16). Matthews H; Ranson M; Kelso MJ Anti-tumour/metastasis effects of the potassium-sparing diuretic amiloride: an orally active anti-cancer drug waiting for its call-of-duty? Int. J. Cancer 2011, 129 (9), 2051-2061. [PubMed: 21544803]

(17). Ranson M; Andronicos NM Plasminogen binding and cancer: promises and pitfalls. Front. Biosci., Landmark Ed 2003, 8, s294-304.

(18). Ngo JCK; Jiang L; Lin Z; Yuan C; Chen Z; Zhang X; Yu H; Wang J; Lin L; Huang M Structural basis for therapeutic intervention of uPA/uPAR system. Curr. Drug Targets 2011, 12 (12), 17291743. [PubMed: 21707478]

(19). Tyndall JD; Kelso MJ; Clingan P; Ranson M Peptides and small molecules targeting the plasminogen activation system: towards prophylactic anti-metastasis drugs for breast cancer. Recent Pat. Anti-Cancer Drug Discovery 2008, 3 (1), 1-13. [PubMed: 18289119]

(20). Nienaber VL; Davidson D; Edalji R; Giranda VL; Klinghofer V; Henkin J; Magdalinos P; Mantei R; Merrick S; Severin JM; Smith RA; Stewart K; Walter K; Wang J; Wendt M; Weitzberg M; Zhao X; Rockway T Structure-directed discovery of potent non-peptidic inhibitors of human urokinase that access a novel binding subsite. Structure 2000, 8 (5), 553-563. [PubMed: 10801494]

(21). Wendt MD; Geyer A; McClellan WJ; Rockway TW; Weitzberg M; Zhao X; Mantei R; Stewart K; Nienaber V; Klinghofer V; Giranda VL Interaction with the S1 beta-pocket of urokinase: 8heterocycle substituted and 6,8-disubstituted 2-naphthamidine urokinase inhibitors. Bioorg. Med. Chem. Lett 2004, 14 (12), 3063-3068. [PubMed: 15149645]

(22). Wendt MD; Rockway TW; Geyer A; McClellan W; Weitzberg M; Zhao X; Mantei R; Nienaber VL; Stewart K; Klinghofer V; Giranda VL Identification of novel binding interactions in the development of potent, selective 2-naphthamidine inhibitors of urokinase. Synthesis, structural analysis, and SAR of N-phenyl amide 6-substitution. J. Med. Chem 2004, 47 (2), 303-324. [PubMed: 14711304]

(23). Bruncko M; McClellan WJ; Wendt MD; Sauer DR; Geyer A; Dalton CR; Kaminski MA; Weitzberg M; Gong J; Dellaria JF; Mantei R; Zhao X; Nienaber VL; Stewart K; Klinghofer V; Bouska J; Rockway TW; Giranda VL $N$-aphtha-midine urokinase plasminogen activator inhibitors with improved pharmacokinetic properties. Bioorg. Med. Chem. Lett 2005, 15 (1), 93 98. [PubMed: 15582418]

(24). Towle MJ; Lee A; Maduakor EC; Schwartz CE; Bridges AJ; Littlefield BA Inhibition of urokinase by 4-substituted benzo[b]thiophene-2-carboxamidines: an important new class of selective synthetic urokinase inhibitor. Cancer Res. 1993, 53 (11), 2553-2559. [PubMed: 8495419]

(25). Rockway TW; Giranda VL Inhibitors of the proteolytic activity of urokinase type plasminogen activator. Curr. Pharm. Des 2003, 9 (19), 1483-1498. [PubMed: 12871064] 
(26). Frederickson M; Callaghan O; Chessari G; Congreve M; Cowan SR; Matthews JE; McMenamin R; Smith DM; Vinkovic M; Wallis NG Fragment-based discovery of mexiletine derivatives as orally bioavailable inhibitors of urokinase-type plasminogen activator. J. Med. Chem 2008, 51 (2), 183-186. [PubMed: 18163548]

(27). Sturzebecher J; Vieweg H; Steinmetzer T; Schweinitz A; Stubbs MT; Renatus M; Wikstrom P 3Amidinophenylalanine-based inhibitors of urokinase. Bioorg. Med. Chem. Lett 1999, 9 (21), 3147-3152. [PubMed: 10560742]

(28). Heinemann V; Ebert MP; Laubender RP; Bevan P; Mala C; Boeck S Phase II randomised proofof-concept study of the urokinase inhibitor upamostat (WX-671) in combination with gemcitabine compared with gemcitabine alone in patients with non-resectable, locally advanced pancreatic cancer. Br. J. Cancer 2013, 108 (4), 766-770. [PubMed: 23412098]

(29). Positive Phase II data for MESUPRON in breast cancer. WILEX Half-Yearly Financial Report 2012; WILEX AG: Munich, Germany, 2012.

(30). Spinar J; Spinarova L; Vitovec J Diuretics in monotherapy and in combination with other diuretics and nondiuretics in the treatment of hypertension. Vnitr. Lek 2013, 59 (6), 486-494. [PubMed: 23808745]

(31). Lant AF; Smith AJ; Wilson GM Clinical evaluation of amiloride, a potassium-sparing diuretic. Clin. Pharmacol. Ther 1969, 10 (1), 50-63. [PubMed: 4884296]

(32). Kleyman TR; Cragoe EJ, Jr. The mechanism of action of amiloride. Semin. Nephrol 1988, 8 (3), 242-248. [PubMed: 2849182]

(33). Palmer BF Regulation of potassium homeostasis. Clin. J. Am. Soc. Nephrol 2015, 10 (6), 10501060. [PubMed: 24721891]

(34). Jankun J; Keck RW; Skrzypczak-Jankun E; Swiercz R Inhibitors of urokinase reduce size of prostate cancer xenografts in severe combined immunodeficient mice. Cancer Res. 1997, 57 (4), 559-563. [PubMed: 9044824]

(35). Vassalli JD; Belin D Amiloride selectively inhibits the urokinase-type plasminogen activator. FEBS Lett. 1987, 214 (1), 187-191. [PubMed: 3106085]

(36). Perazella MA Drug-induced hyperkalemia: old culprits and new offenders. Am. J. Med 2000, 109 (4), 307-314. [PubMed: 10996582]

(37). Wermuth CG Selective optimization of side activities: another way for drug discovery. J. Med. Chem 2004, 47 (6), 1303-1314. [PubMed: 14998318]

(38). Matthews H; Ranson M; Tyndall JD; Kelso MJ Synthesis and preliminary evaluation of amiloride analogs as inhibitors of the urokinase-type plasminogen activator (uPA). Bioorg. Med. Chem. Lett 2011, 21 (22), 6760-6766. [PubMed: 21978672]

(39). Kleyman TR; Cragoe EJ, Jr. Amiloride and its analogs as tools in the study of ion transport. J. Membr. Biol 1988, 105 (1), 1-21. [PubMed: 2852254]

(40). Song CW; Lyons JC; Makepeace CM; Griffin RJ; Cragoe EJ, Jr. Effects of HMA, an analog of amiloride, on the thermosensitivity of tumors in vivo. Int. J. Radiat. Oncol., Biol., Phys 1994, 30 (1), 133-139. [PubMed: 8083106]

(41). Luo J; Tannock IF Inhibition of the regulation of intracellular $\mathrm{pH}$ : potential of 5-(N,Nhexamethylene) amiloride in tumour-selective therapy. Br. J. Cancer 1994, 70 (4), 617-624. [PubMed: 7917906]

(42). Rich IN; Worthington-White D; Garden OA; Musk P Apoptosis of leukemic cells accompanies reduction in intracellular $\mathrm{pH}$ after targeted inhibition of the $\mathrm{Na}(+) / \mathrm{H}(+)$ exchanger. Blood 2000, 95 (4), 1427-1434. [PubMed: 10666221]

(43). Man CH; Lam SS; Sun MK; Chow HC; Gill H; Kwong YL; Leung AY A novel tescalcin-sodium/ hydrogen exchange axis underlying sorafenib resistance in FLT3-ITD+ AML. Blood 2014, 123 (16), 2530-2539. [PubMed: 24608976]

(44). Ye JH; Gao J; Wu YN; Hu YJ; Zhang CP; Xu TL Identification of acid-sensing ion channels in adenoid cystic carcinomas. Biochem. Biophys. Res. Commun 2007, 355 (4), 986-992. [PubMed: 17324378]

(45). Rowson-Hodel AR; Berg AL; Wald JH; Hatakeyama J; VanderVorst K; Curiel DA; Leon LJ; Sweeney C; Carraway KL Hexamethylene amiloride engages a novel reactive oxygen species- 
and lysosome-dependent programmed necrotic mechanism to selectively target breast cancer cells. Cancer Lett. 2016, 375 (1), 62-72. [PubMed: 26944316]

(46). Kleyman TR; Cragoe EJ, Jr. Cation transport probes: the amiloride series. Methods Enzymol. 1990, 191, 739-755. [PubMed: 1963659]

(47). Cragoe EJ; Woltersdorf OW; Bicking JB; Kwong SF; Jones JH Pyrazine Diuretics. II. NAmidino-3-amino-5-substituted 6Halopyrazinecarboxamides. J. Med. Chem 1967, 10 (1), 66-75. [PubMed: 6031705]

(48). Patwardhan NN; Ganser LR; Kapral GJ; Eubanks CS; Lee J; Sathyamoorthy B; Al-Hashimi HM; Hargrove AE Amiloride as a new RNA-binding scaffold with activity against HIV-1 TAR. MedChemComm 2017, 8 (5), 1022-1036. [PubMed: 28798862]

(49). Zeslawska E; Schweinitz A; Karcher A; Sondermann P; Sperl S; Sturzebecher J; Jacob U Crystals of the urokinase type plasminogen activator variant beta(c)-uPAin complex with small molecule inhibitors open the way towards structure-based drug design. J. Mol. Biol 2000, 301 (2), 465-475. [PubMed: 10926521]

(50). Lee S; Yi KY; Hwang SK; Lee BH; Yoo S.-e.; Lee K (5-Arylfuran-2-ylcarbonyl)guanidines as cardioprotectives through the inhibition of $\mathrm{Na}+\mathrm{H}+$ exchanger isoform-1. J. Med. Chem 2005, 48 (8), 2882-2891. [PubMed: 15828827]

(51). Cochran BJ; Gunawardhana LP; Vine KL; Lee JA; Lobov S; Ranson M The CD-loop of PAI-2 (SERPINB2) is redundant in the targeting, inhibition and clearance of cell surface uPA activity. BMC Biotechnol. 2009, 9, 43. [PubMed: 19442270]

(52). Yung-Chi C; Prusoff WH Relationship between the inhibition constant (KI) and the concentration of inhibitor which causes 50\% inhibition (I50) of an enzymatic reaction. Biochem. Pharmacol 1973, 22 (23), 3099-3108. [PubMed: 4202581]

(53). Nienaber V; Wang J; Davidson D; Henkin J Re-engineering of human urokinase provides a system for structure-based drug design at high resolution and reveals a novel structural subsite. J. Biol. Chem 2000, 275 (10), 7239-7248. [PubMed: 10702294]

(54). Bachovchin DA; Koblan LW; Wu W; Liu Y; Li Y; Zhao P; Woznica I; Shu Y; Lai JH; Poplawski SE; Kiritsy CP; Healey SE; DiMare M; Sanford DG; Munford RS; Bachovchin WW; Golub TR A high-throughput, multiplexed assay for superfamily-wide profiling of enzyme activity. Nat. Chem. Biol 2014, 10 (8), 656-663. [PubMed: 24997602]

(55). Soisson SM; Patel SB; Abeywickrema PD; Byrne NJ; Diehl RE; Hall DL; Ford RE; Reid JC; Rickert KW; Shipman JM; Sharma S; Lumb KJ Structural definition and substrate specificity of the S28 protease family: the crystal structure of human prolylcarboxypeptidase. BMC Struct. Biol 2010, 10 (16), 16. [PubMed: 20540760]

(56). Soudijn W; Van Wijngaarden I; Ijzermann AP Allosteric modulation of G protein-coupled receptors: perspectives and recent developments. Drug Discovery Today 2004, 9 (17), 752-758. [PubMed: 15450241]

(57). Gao ZG; Ijzerman AP Allosteric modulation of A(2A) adenosine receptors by amiloride analogues and sodium ions. Biochem. Pharmacol 2000, 60 (5), 669-676. [PubMed: 10927025]

(58). Garritsen A; Beukers MW; Ijzerman AP; Soudijn W Recognition of adenosine receptors by amiloride and its analogues. Nucleosides Nucleotides 1991, 10 (5), 1107-1111.

(59). Garritsen A; Ijzerman AP; Tulp M. M. Th.; Cragoe EJ, Jr.; Soudijn W Receptor binding profiles of amiloride analogues provide no evidence for a link between receptors and the $\mathrm{Na}+\mathrm{H}+$ exchanger, but indicate a common structure on receptor proteins. J. Recept. Res 1991, 11 (6), 891-907. [PubMed: 1661335]

(60). de Lera Ruiz M; Lim Y-H; Zheng J Adenosine A2A receptor as a drug discovery target. J. Med. Chem 2014, 57 (9), 3623-3650. [PubMed: 24164628]

(61). Massink A; Louvel J; Adlere I; van Veen C; Huisman BJ; Dijksteel GS; Guo D; Lenselink EB; Buckley BJ; Matthews H; Ranson M; Kelso M; Ijzerman AP 5'-Substituted amiloride derivatives as allosteric modulators binding in the sodium ion pocket of the adenosine A2A receptor. J. Med. Chem 2016, 59 (10), 4769-4777. [PubMed: 27124340]

(62). Klinghofer V; Stewart K; McGonigal T; Smith R; Sarthy A; Nienaber V; Butler C; Dorwin S; Richardson P; Weitzberg M; Wendt M; Rockway T; Zhao X; Hulkower KI; Giranda VL Species 
specificity of amidine-based urokinase inhibitors. Biochemistry 2001, 40 (31), 9125-9131. [PubMed: 11478879]

(63). McNay JL; Oran E Possible predisposition of diabetic patients to hyperkalemia following administration of potassium-retaining diuretic, amiloride (MK 870). Metab., Clin. Exp 1970, 19 (1), 58-70. [PubMed: 5410663]

(64). Baer JE; Jones CB; Spitzer SA; Russo HF The potassium-sparing and natriuretic activity of Namidino-3,5-diamino-6-chloropyrazinecarboxamide hydro-chloride dihydrate (amiloride hydrochloride). J. Pharmacol. Exp. Ther 1967, 157 (2), 472-485. [PubMed: 6039836]

(65). Comprehensive pharmacokinetic profiling of compounds 18 and 26 will be reported elsewhere.

(66). Uhland K; Siphos B; Arkona C; Schuster M; Petri B; Steinmetzer P; Mueller F; Schweinitz A; Steinmetzer T; Van De Locht A Use of IHC and newly designed matriptase inhibitors to elucidate the role of matriptase in pancreatic ductal adenocarcinoma. Int. J. Oncol 2009, 35 (2), 347-357. [PubMed: 19578749]

(67). Pothula SP; Xu Z; Goldstein D; Biankin AV; Pirola RC; Wilson JS; Apte MV Hepatocyte growth factor inhibition: a novel therapeutic approach in pancreatic cancer. Br. J. Cancer 2016, 114 (3), 269-280. [PubMed: 26766740]

(68). Nikishkin NI; Huskens J; Verboom W Transition metal-catalyzed functionalization of pyrazines. Org. Biomol. Chem 2013, 11 (22), 3583-3602. [PubMed: 23632914]

(69). Chessell IP; Michel AD; Humphrey PP Properties of the pore-forming P2 $\times 7$ purinoceptor in mouse NTW8 microglial cells. Br. J. Pharmacol 1997, 121 (7), 1429-1437. [PubMed: 9257924]

(70). Amith SR; Wilkinson JM; Fliegel L KR-33028, a potent inhibitor of the $\mathrm{Na}(+) / \mathrm{H}(+)$ exchanger NHE1, suppresses metastatic potential of triple-negative breast cancer cells. Biochem. Pharmacol 2016, 118, 31-39. [PubMed: 27521504]

(71). Rosenberg MR; Weaver LM; Casarotto MG Probing interactions of Vpu from HIV-1 with amiloride-based compounds. Biochim. Biophys. Acta, Biomembr 2016, 1858 (4), 733-739.

(72). Jalily PH; Eldstrom J; Miller SC; Kwan DC; Tai SS-H; Chou D; Niikura M; Tietjen I; Fedida D Mechanisms of action of novel influenza A/M2 viroporin inhibitors derived from hexamethylene amiloride. Mol. Pharmacol 2016, 90 (2), 80-95. [PubMed: 27193582]

(73). Heitman LH; Ye K; Oosterom J; Ijzerman AP Amiloride derivatives and a nonpeptidic antagonist bind at two distinct allosteric sites in the human gonadotropin-releasing hormone receptor. Mol. Pharmacol 2008, 73 (6), 1808-1815. [PubMed: 18344315]

(74). Snell HD; Gonzales EB 5-(N, N-Hexamethylene) amiloride is a GABA-A $\rho 1$ receptor positive allosteric modulator. Channels 2016, 10 (6), 498-506. [PubMed: 27367557]

(75). Leng TD; Si HF; Li J; Yang T; Zhu M; Wang B; Simon RP; Xiong ZG Amiloride analogs as ASIC1a inhibitors. CNS Neurosci. Ther 2016, 22 (6), 468-476. [PubMed: 26890278]

(76). Walsh KB Targeting GIRK channels for the development of new therapeutic agents. Front. Pharmacol 2011, 2, 64. [PubMed: 22059075]

(77). Cox G; Ewart G; Gage P A Method of Modulating Ion Channel Functional Activity. PCT Int. Appl WO2000021538 A1, 2000.

(78). Zhao G; Yuan C; Wind T; Huang Z; Andreasen PA; Huang M Structural basis of specificity of a peptidyl urokinase inhibitor, upain-1. J. Struct. Biol 2007, 160 (1), 1-10. [PubMed: 17692534]

(79). Otwinowski Z; Minor W Processing of X-ray diffraction data collected in oscillation mode. Methods Enzymol. 1997, 276, 307-326.

(80). The CCP4 suite: Programs for protein crystallography. Collaborative Computational Project, Number 4. Acta Crystallogr., Sect. D: Biol. Crystallogr 1994, 50, 760-763, [PubMed: 15299374]

(81). Jiang L; Botkjaer KA; Andersen LM; Yuan C; Andreasen PA; Huang M Rezymogenation of active urokinase induced by an inhibitory antibody. Biochem. J 2013, 449 (1), 161-166. [PubMed: 23016918]

(82). Emsley P; Cowtan K Coot: model-building tools for molecular graphics. Acta Crystallogr., Sect. D: Biol. Crystallogr 2004, 60, 2126-2132. [PubMed: 15572765]

(83). Lu M; Echeverri F; Kalabat D; Laita B; Dahan DS; Smith RD; Xu H; Staszewski L; Yamamoto J; Ling J; Hwang N; Kimmich R; Li P; Patron E; Keung W; Patron A; Moyer BD Small molecule activator of the human epithelial sodium channel. J. Biol. Chem 2008, 283 (18), 11981-11994. [PubMed: 18326490] 
(84). Xu Z; Vonlaufen A; Phillips PA; Fiala-Beer E; Zhang X; Yang L; Biankin AV; Goldstein D; Pirola RC; Wilson JS; Apte MV Role of pancreatic stellate cells in pancreatic cancer metastasis. Am. J. Pathol 2010, 177 (5), 2585-2596. [PubMed: 20934972] 

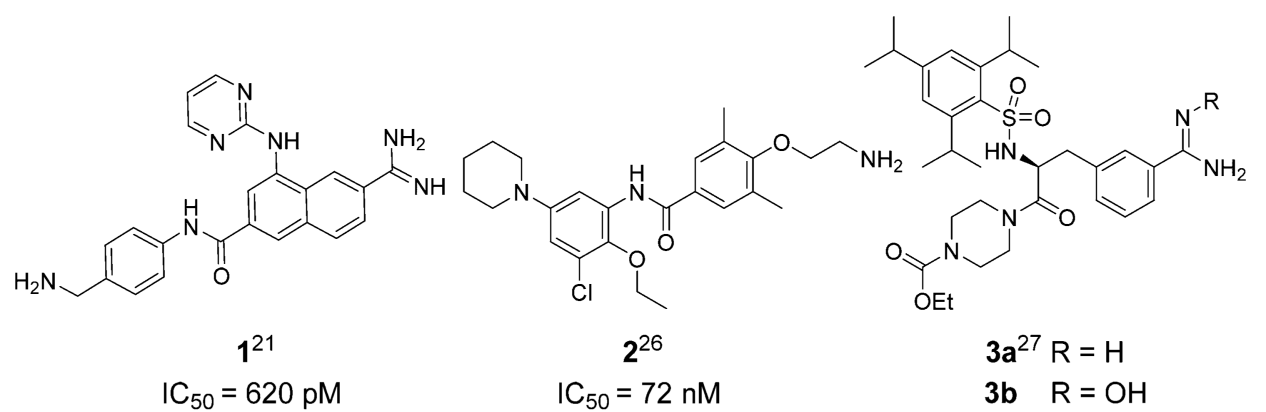

$\mathrm{C}_{50}=620 \mathrm{pM}$

$72 \mathrm{nM}$

$3 \mathrm{a}^{27} \mathrm{R}=\mathrm{H}$

3b $\quad \mathrm{R}=\mathrm{OH}$<smiles>N=C(N)NC(=O)c1nc(Cl)c(N)n1N</smiles><smiles>N=C(N)NC(=O)c1nc(Cl)c(N2CCCCCC2)nc1N</smiles>
$K_{i}=410 \mathrm{nM}$

Amiloride $\mathbf{4}^{35}$

$\mathrm{IC}_{50}=7 \mu \mathrm{M}$

5-Hexamethylene amiloride $5^{38}$

HMA IC ${ }_{50}=6 \mu \mathrm{M}$

Figure 1.

Structures of reported uPA inhibitors. 


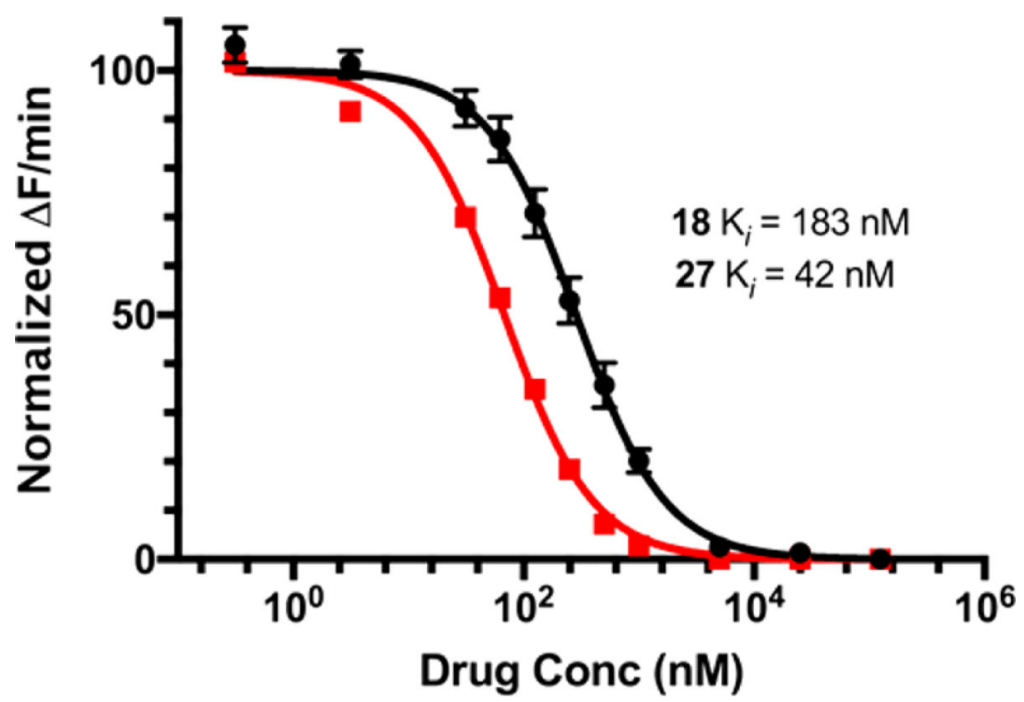

Figure 2.

Inhibition of low molecular weight human uPA by 18 (black) and 27 (red). Data points represent the mean $\pm \operatorname{SEM}(n=3)$ from a single representative experiment. 


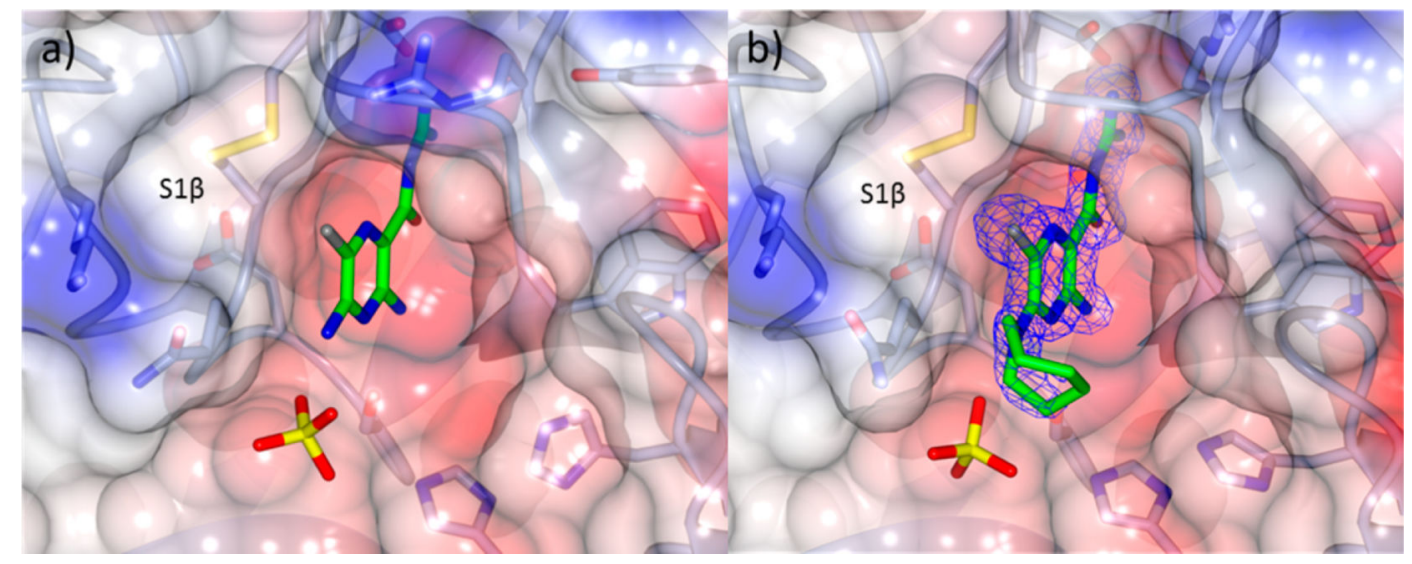

Figure 3.

X-ray cocrystal structures of (a) amiloride $4^{49}$ (PDB code 1F5L) and (b) HMA 5 (PDB code 5ZA7, $1.7 \AA$ A resolution) bound to uPA. Blue wire basket $=2 m F_{o}-D F_{C}$ map contoured at $1.5 \sigma$. 


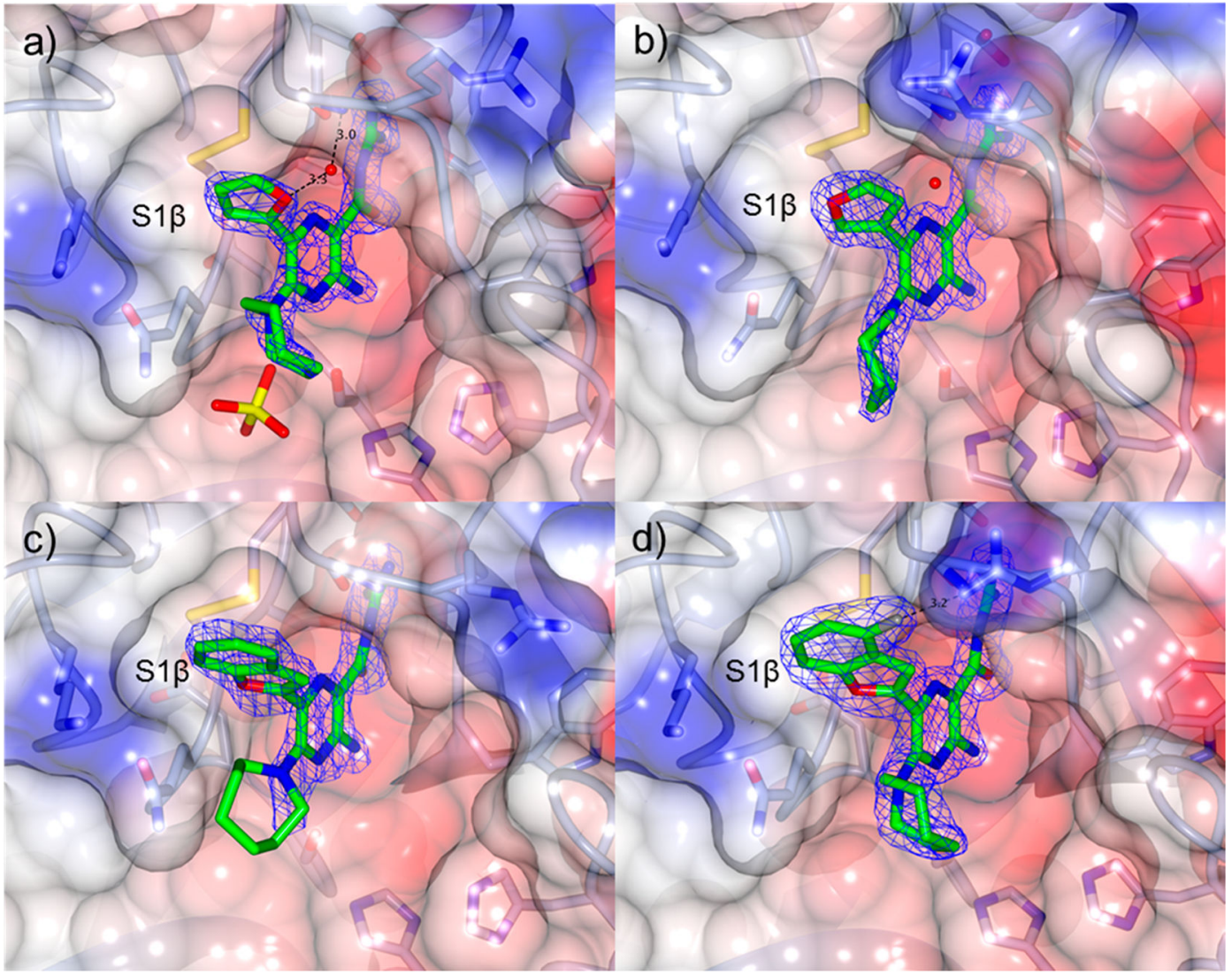

Figure 4.

X-ray cocrystal structures of: (a) 22 (PDB code 5ZAE, $1.7 \AA$ ), (b) 23 (PDB code 5ZAF, 1.7 $\AA$ ), (c) $\mathbf{1 8}$ (PDB code 5ZA9, $1.6 \AA$ ), and (d) $\mathbf{1 9}$ (PDB code 5ZC5, $1.9 \AA$ ) bound to uPA. Blue wire baskets represent $2 m F_{o}-D F_{C}$ maps contoured at $1.5 \sigma$. 


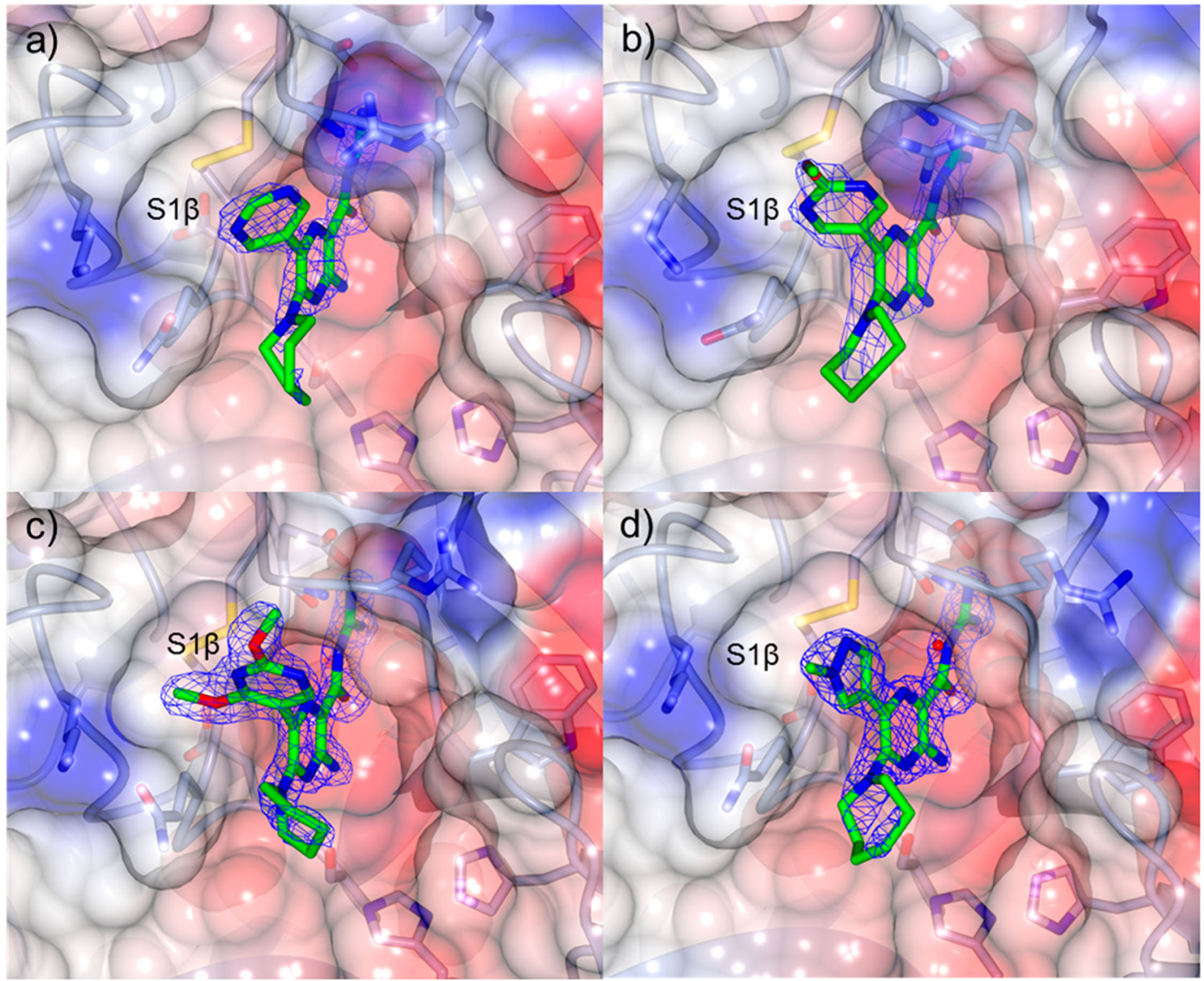

Figure 5.

X-ray cocrystal structures of (a) 25 (PDB code 5ZAG, $2.0 \AA$ ), (b) 26 (PDB code 5ZAH, 3.0 $\AA$ ), (c) 27 (PDB code 5ZAJ, $1.7 \AA$ ), and (d) 14 (PDB code 5ZA8, $1.9 \AA$ ) bound to uPA. Blue wire baskets represent $2 m F_{o}-D F_{C}$ maps contoured at $1.5 \sigma$. 


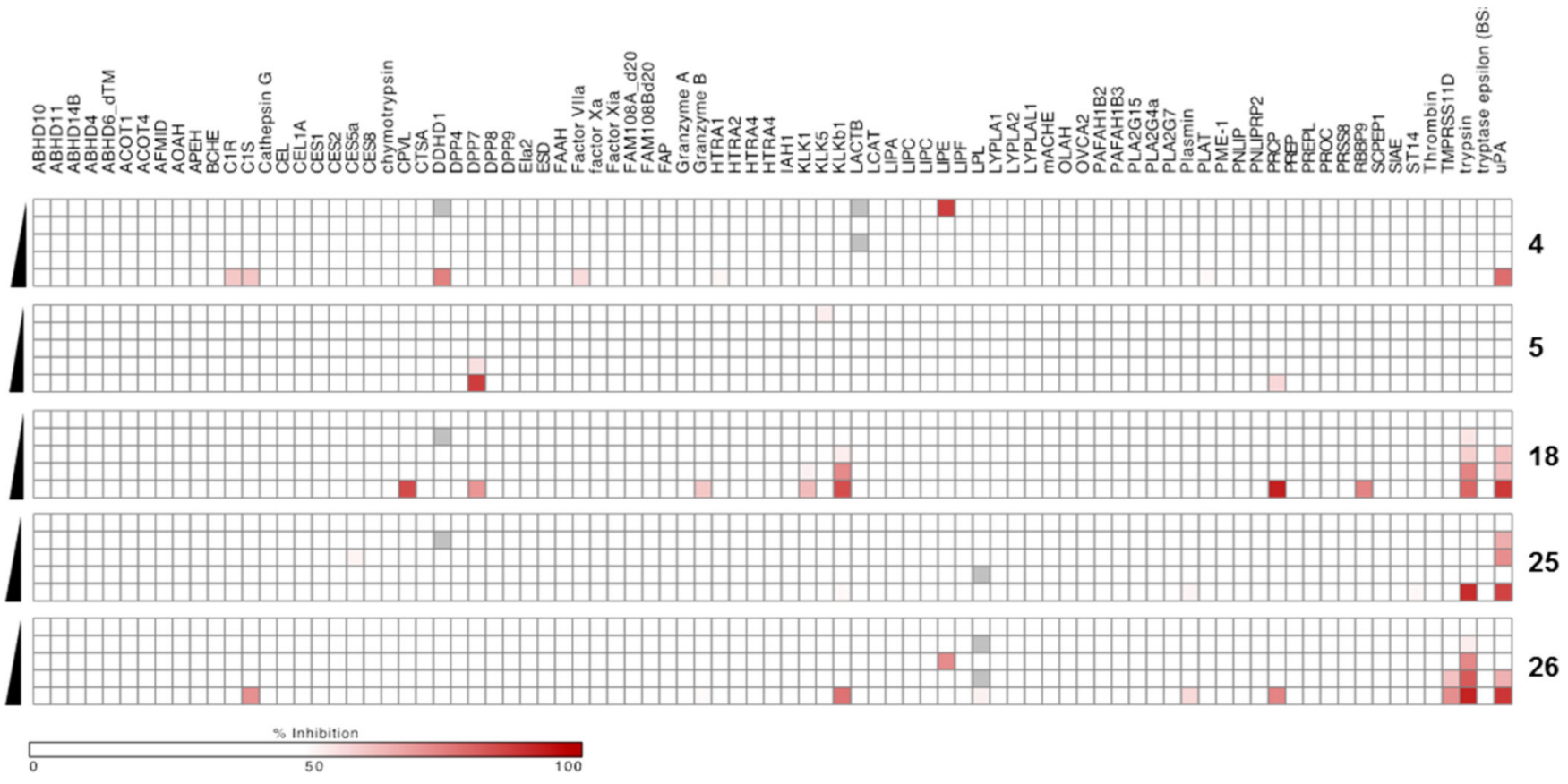

Figure 6.

EnPlex $^{54}$ screening of amiloride 4, HMA 5 and analogs 18, 25, and 26 for inhibition of 85 serine hydrolases. Gray boxes indicate wells in which no fluorescence was observed. The singular readouts suggesting activity of $\mathbf{4}$ and $\mathbf{2 6}$ against LIPE are artifacts. Black arrows indicate decreasing compound concentration from $33.3 \mu \mathrm{M}$ to $10 \mathrm{nM}$. 


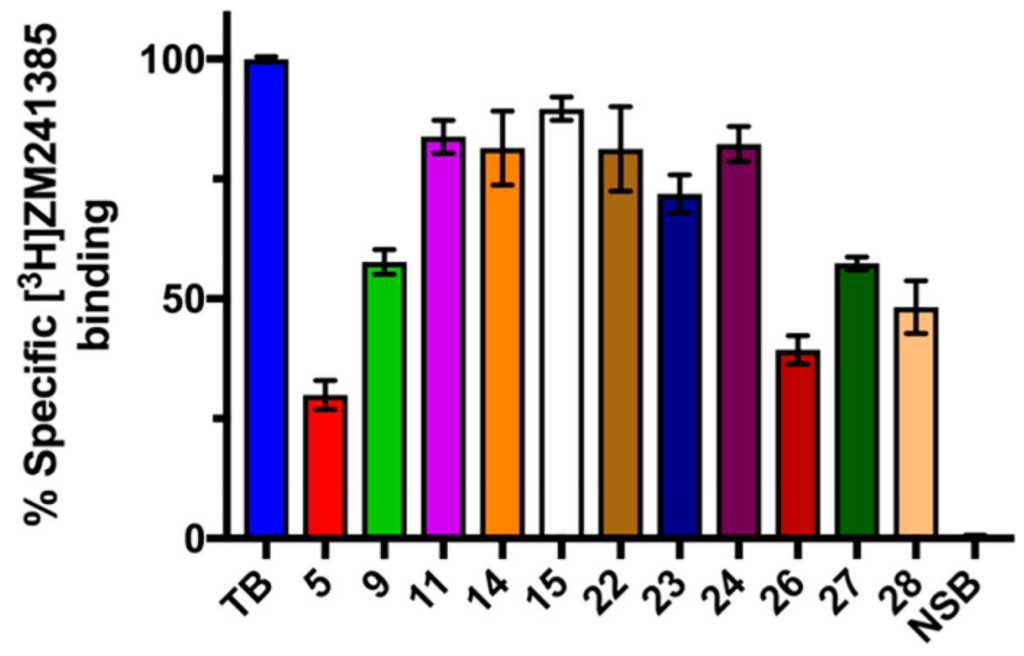

Figure 7.

Single concentration screening $(10 \mu \mathrm{M})$ of HMA 5 and 6-substituted HMA analogs as antagonists of the human adenosine $\mathrm{A}_{2 \mathrm{~A}}$ receptor $\left(\mathrm{h} \mathrm{A}_{2 \mathrm{~A}} \mathrm{AR}-\mathrm{WT}\right)$. Radioligand

$\left[{ }^{3} \mathrm{H}\right] \mathrm{ZM} 241385$ was present at $2.5 \mathrm{nM}$. Total binding (TB, 100\%) of $\left[{ }^{3} \mathrm{H}\right] \mathrm{ZM} 241385$ was determined in the absence of inhibitors. Nonspecific binding (NSB, 0\%) was determined in the presence of $100 \mu \mathrm{M} \mathrm{A}_{2 \mathrm{~A}}$ agonist $5^{\prime}$-( $N$-ethylcarboxamido)adenosine (NECA). Data represent the mean \pm SEM from at least three independent experiments conducted in duplicate. Methods were as described in ref 61. 


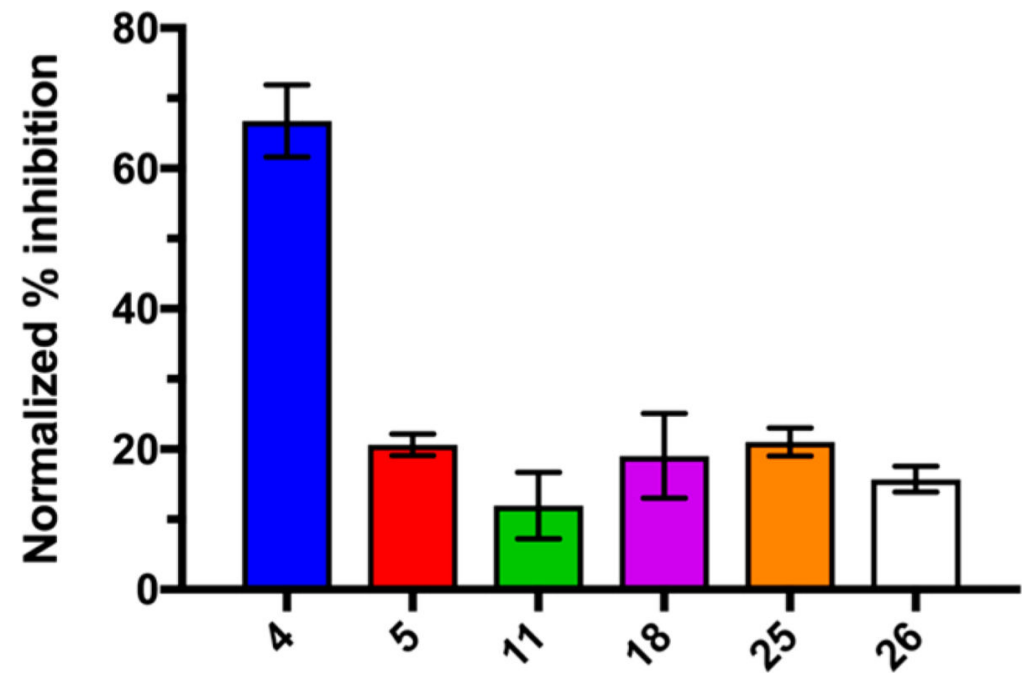

Figure 8.

Inhibition of ENaC activity by amiloride 4, HMA 5 and 6-substituted analogs 11, 18, 25, and $\mathbf{2 6}$ in transfected HEK-293 cells. All compounds were present at $10 \mu \mathrm{M}$. Data represent the mean $\pm \mathrm{SD}(n=4)$. 

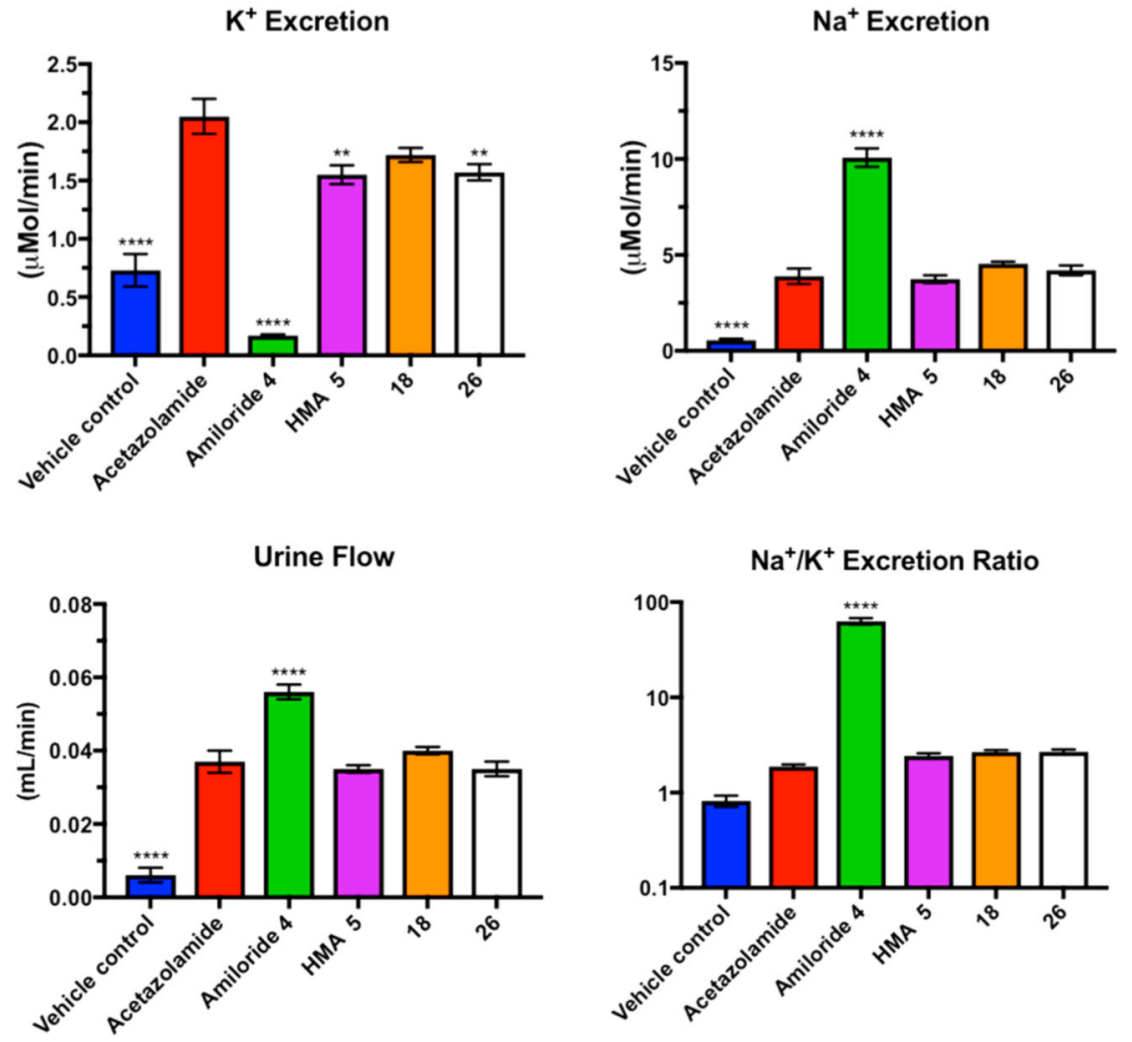

Figure 9.

Effects of amiloride 4, HMA 5, and 6-substituted analogs 18 and 26 on urine flow and excretion of $\mathrm{Na}^{+}$and $\mathrm{K}^{+}$in Sprague Dawley rats. Rats were dosed with $25 \mathrm{mg} / \mathrm{kg}$ acetazolamide prior to administering $1.5 \mathrm{mg} / \mathrm{kg}$ of test compound. Data represent the mean \pm SEM $(n=8):(* *) \mathrm{p}<0.001,(* * * *) p<0.0001$ relative to acetazolamide control. 


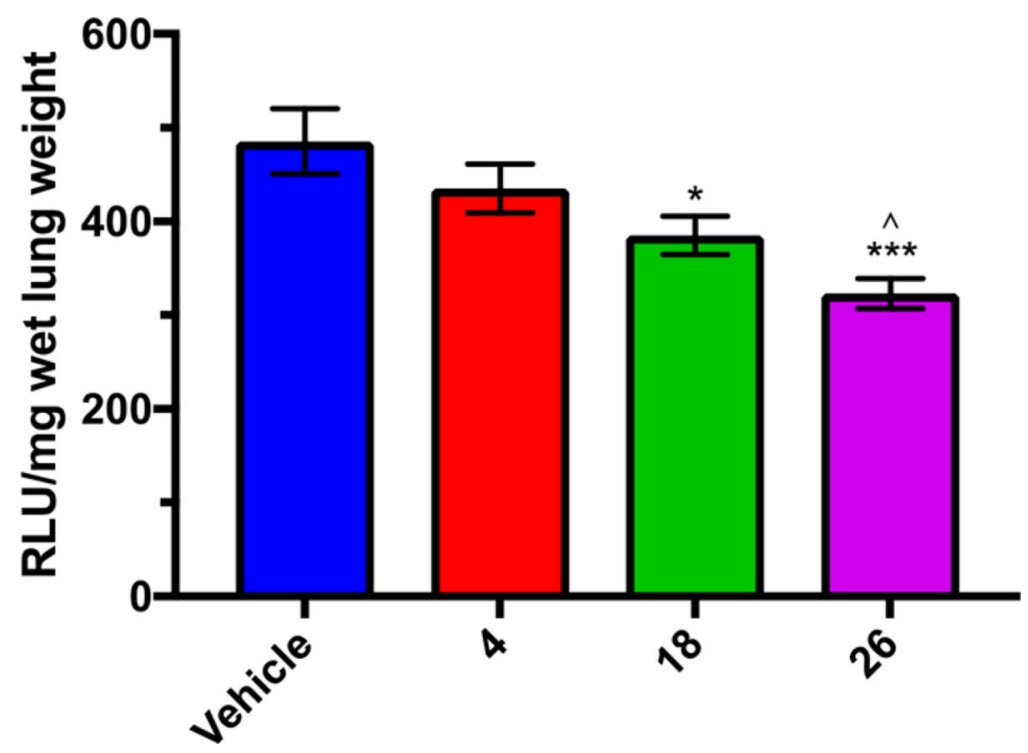

Figure 10.

Effects of amiloride 4, 18, and 26 in experimental mouse model of late-stage lung metastasis. All compounds were administered once daily at $7.5 \mathrm{mg} \mathrm{kg}^{-1} \mathrm{day}^{-1}$ for 21 days. Lung metastasis was quantified by end point luciferase assay of lung homogenates. Data represent the mean \pm SEM (18 and $26 n=6$; vehicle and $4 n=4)$ : (*) p < 0.03; (***) $p<$ 0.001 relative to vehicle control, $(\wedge) p<0.03$ relative to 4 . 
A)

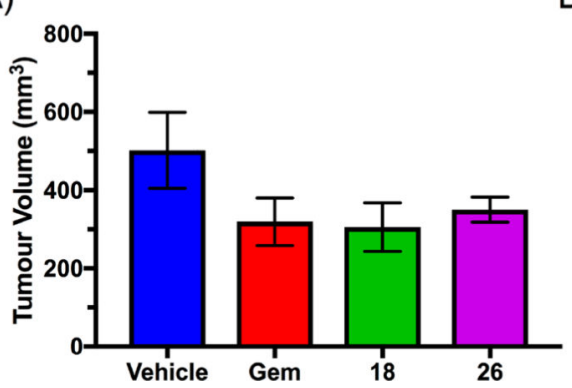

B)

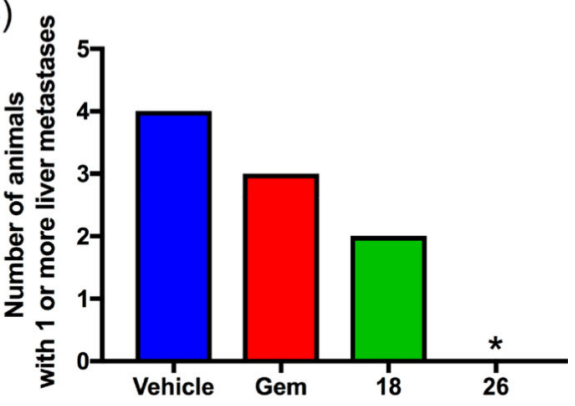

Figure 11.

(A) Effects of gemcitabine (Gem), 18, and $\mathbf{2 6}$ on end point primary tumor volume in an orthotopic xenograft pancreatic cancer model. Mice received oral gemcitabine $75 \mathrm{mg} / \mathrm{kg}$ twice weekly or daily intraperitoneal injections of 18 or $26(10 \mathrm{mg} / \mathrm{kg})$ for 28 days. Data represent the mean \pm SEM ( $n=4$ per group). (B) Number of mice with histologically confirmed liver metastases after receiving gemcitabine, 18, and $\mathbf{2 6}$ ( $n=4$ per group): (*) $p=$ 0.0285 vs vehicle, $\chi^{2}$ test. 


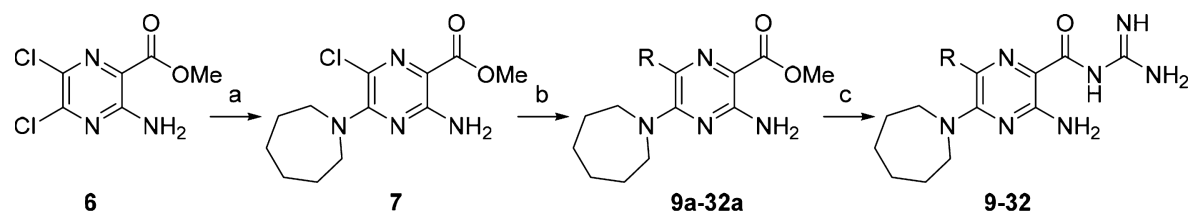

Scheme 1. Synthesis of 6-(Hetero)aryl HMA Analogs 9-32 ${ }^{\mathrm{a}}$

${ }^{a}$ Reagents and conditions: (a) $\left(\mathrm{CH}_{2}\right)_{6} \mathrm{NH}$, DIPEA, ${ }^{i} \mathrm{PrOH}$, reflux, $2 \mathrm{~h}, 86 \%$; (b) $\mathrm{R}-\mathrm{B}(\mathrm{OH})_{2}$ or $\mathrm{R}-\mathrm{B}$ (pin), $\mathrm{Pd}\left(\mathrm{PPh}_{3}\right)_{4}, \mathrm{~K}_{2} \mathrm{CO}_{3}$, toluene/MeOH (4:1), reflux, 0.5-18 h; (c) guanidine (2 $\mathrm{M}$ in $\mathrm{MeOH}), \mathrm{DMF}, \mathrm{rt}, 18 \mathrm{~h}$. 


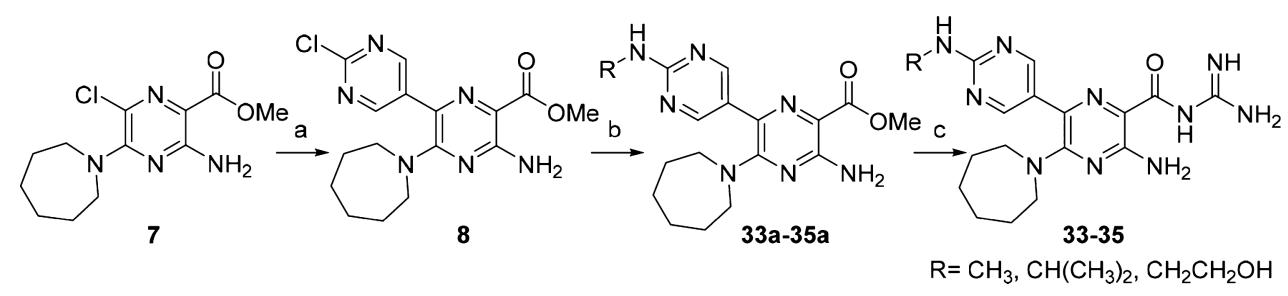

Scheme 2. Synthesis of 6-(2-(Alkylamino)pyrimidin-5-yl) Analogs 33-35a

${ }^{a}$ Reagents and conditions: (a) (2-chloropyrimidin-5-yl)boronic acid, $\mathrm{Pd}\left(\mathrm{PPh}_{3}\right)_{4}, \mathrm{~K}_{2} \mathrm{CO}_{3}$, toluene/MeOH (4:1), reflux, 18 h, 64\%; (b) (i) R-NH $\mathrm{NH}_{2}$, DIPEA, DMF, rt, 2 d or (ii) $\mathrm{R}-\mathrm{NH}_{2}$, DIPEA, $i$-PrOH, reflux, 4 h, 83-87\%; (c) guanidine (2 M in MeOH), DMF, rt, 18 h, $85-89 \%$. 


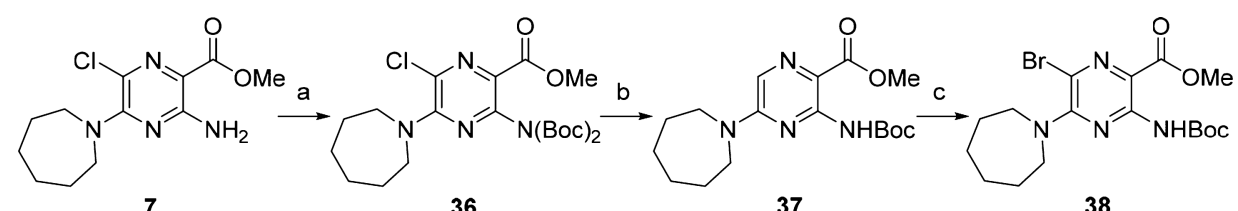

7

36

37

38

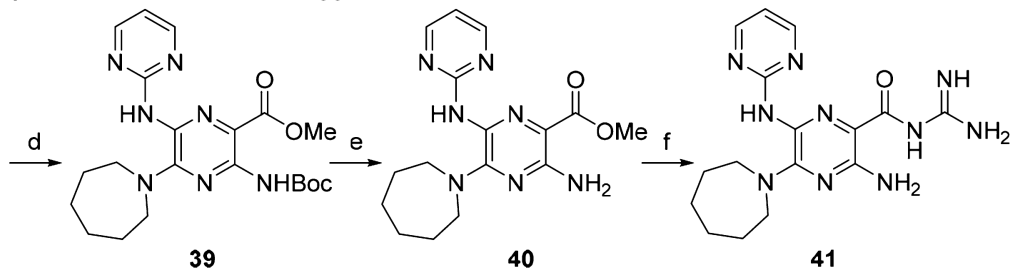

Scheme 3. Synthesis of 2-Aminopyrimidinyl Derivative $41^{\mathrm{a}}$

${ }^{a}$ Reagents and conditions: (a) (Boc) $)_{2} \mathrm{O}, \mathrm{CH}_{2} \mathrm{Cl}_{2}$, DIPEA, DMAP, rt, 16 h, 85\%; (b) $\mathrm{Pd} / \mathrm{C}, \mathrm{H}_{2}$ (1 atm), $\mathrm{MgO}, \mathrm{MeOH}, \mathrm{rt}, 48 \mathrm{~h}, 92 \%$; (c) $\mathrm{N}$-bromosuccinimide, $\mathrm{CH}_{2} \mathrm{Cl}_{2}, \mathrm{rt}, 5 \mathrm{~h}, 60 \%$; (d) 2aminopyrimidine, $\mathrm{Pd}_{2}(\mathrm{dba})_{3} / \mathrm{Xantphos,}$,4-dioxane, $\mathrm{Cs}_{2} \mathrm{CO}_{3}, 100{ }^{\circ} \mathrm{C}, 18 \mathrm{~h}, 30 \%$; (e) TFA, $\mathrm{CH}_{2} \mathrm{Cl}_{2}, \mathrm{rt}, 16 \mathrm{~h}, 98 \%$; (f) guanidine (2 $\mathrm{M}$ in $\mathrm{MeOH}$ ), DMF, rt, $35 \%$. 
Table 1.

Inhibition of uPA by 6-Substituted HMA Analogs ${ }^{a}$
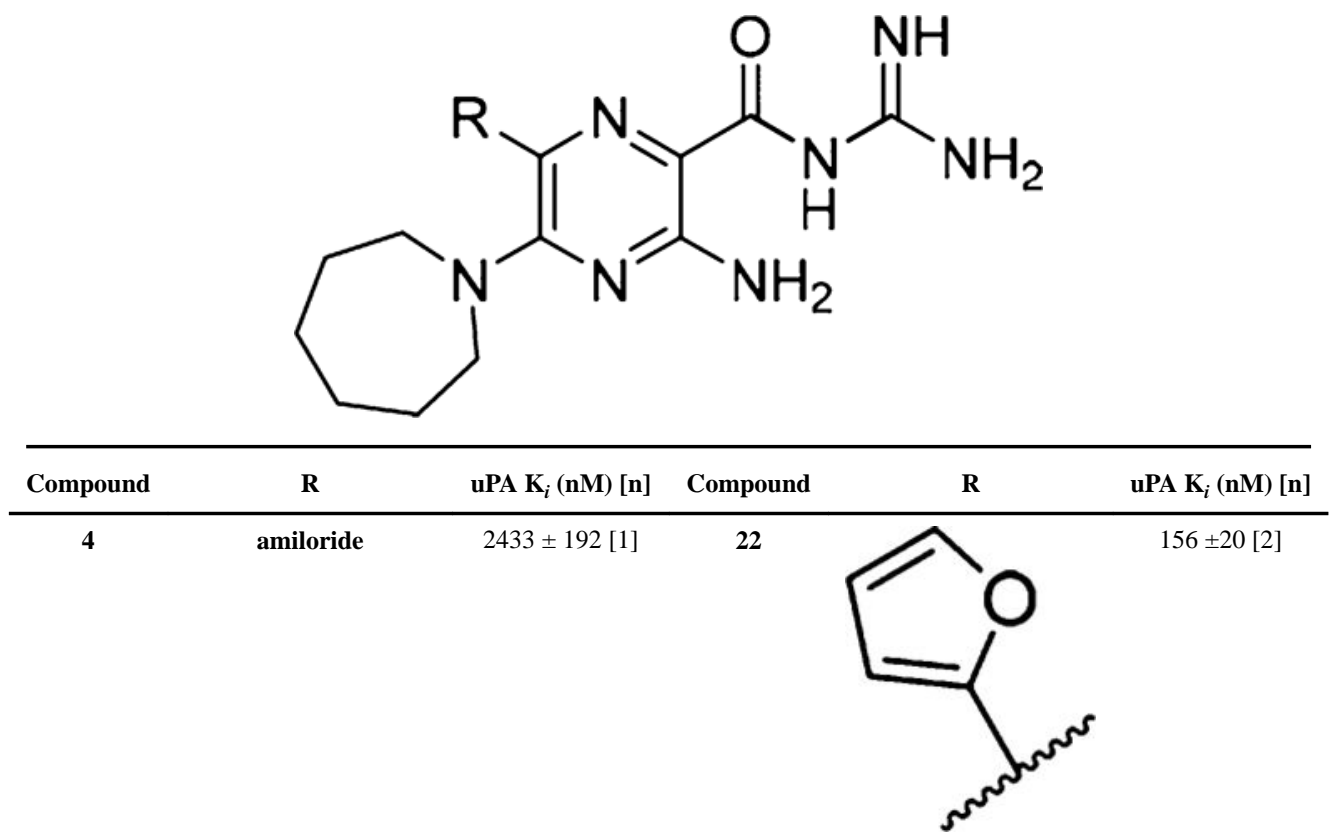

5<smiles>CC(Cl)C1CC1</smiles>

9<smiles>CC(C)(C)c1ccccc1</smiles>

$5020 \pm 1750[1]$

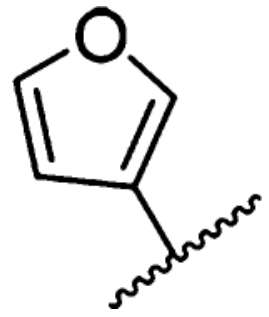

10

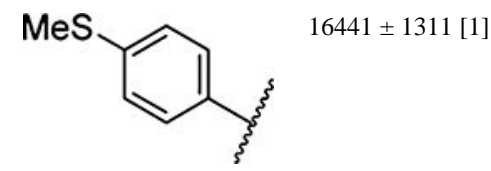

24<smiles>CC(C)c1cccnc1F</smiles>

25<smiles>CC(C)c1cncnc1</smiles>

23

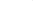

$166 \pm 34$ [2]

$1778 \pm 249[1]$

$108 \pm 17$ [2] 


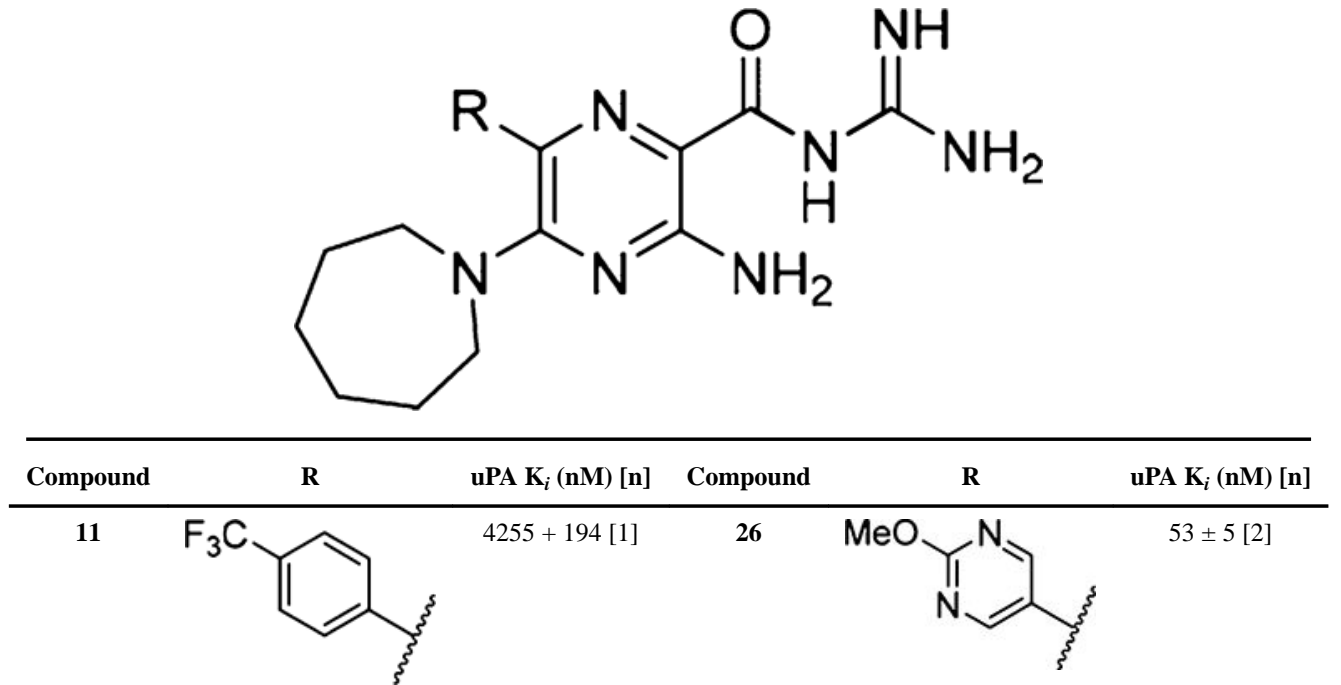<smiles>CC(C)(C)c1ccc2c(c1)OCO2</smiles>

27<smiles>COc1ncc(C(C)C)c(OC)n1</smiles>

$42 \pm 2[4]$

13<smiles>CC(C)c1cncc2ccccc12</smiles>

$3539 \pm 192$ [2]

28<smiles>CCCCc1ccc(OC)nc1OC</smiles>

$884 \pm 14$ [2]

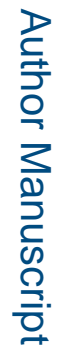

14

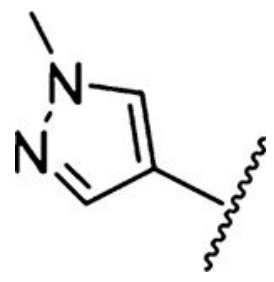

$508 \pm 9$ [2]

29<smiles>COc1ccc(C(C)C)c(OC)c1</smiles>

30<smiles>COc1cc(OC)cc(C(C)(C)C)c1</smiles>

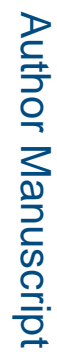

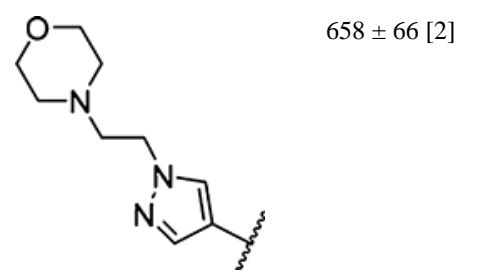

JMed Chem. Author manuscript; available in PMC 2019 September 27. 


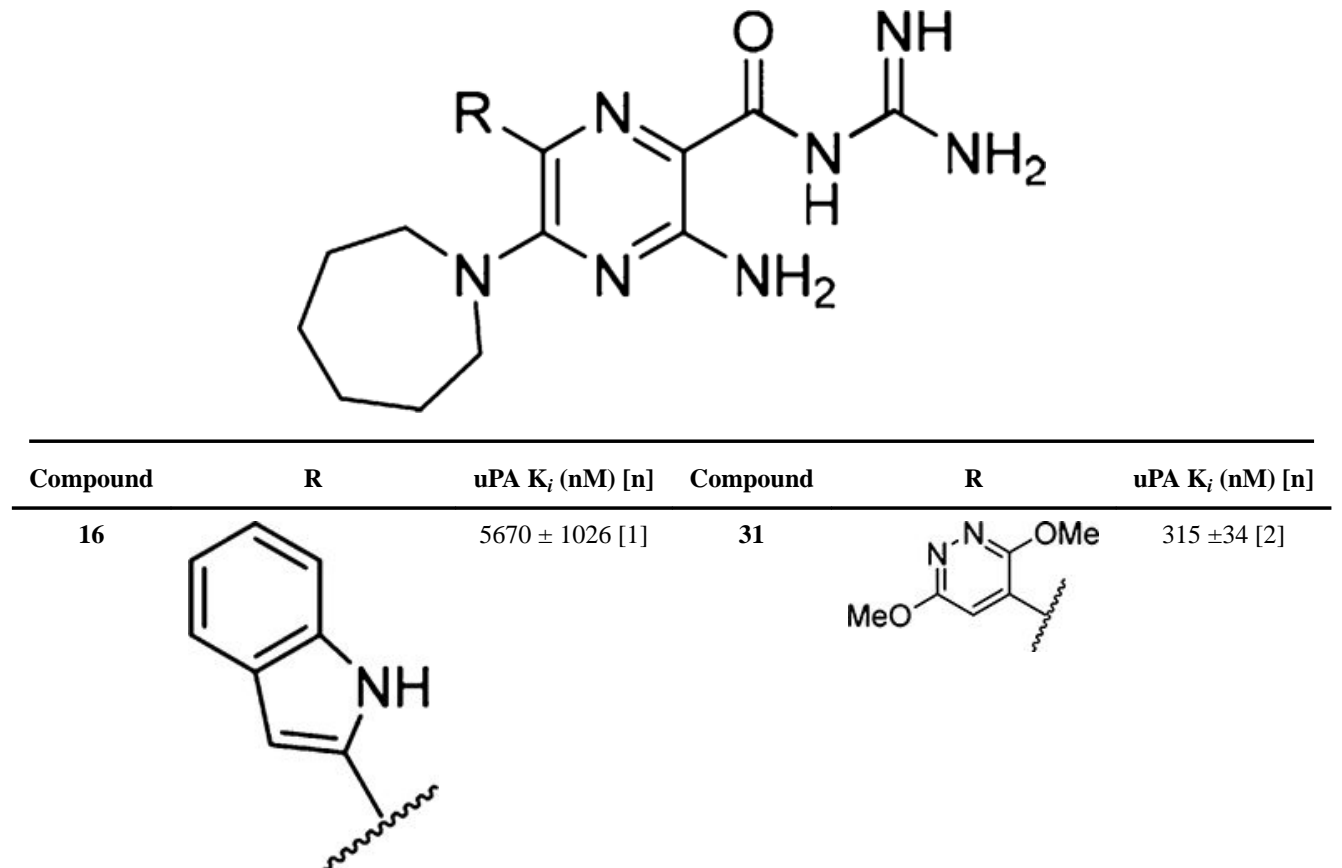

17<smiles>CC(C)c1cc2ccccc2s1</smiles>

18<smiles>CC(C)c1cc2ccccc2o1</smiles>

19<smiles>CC(C)c1cc2c(F)cccc2o1</smiles>

$3308 \pm 300[1]$

32<smiles>CC(C)c1cnc(N)nc1</smiles>

$183 \pm 10[4]$

33<smiles>CNc1ncc(C(C)C)cn1</smiles>

$88 \pm 33$ [2]

34<smiles>CC(C)Nc1ncc(C(C)C)cn1</smiles>

$21 \pm 3$ [2]

$43 \pm 4[2]$

$42 \pm 4[2]$ 


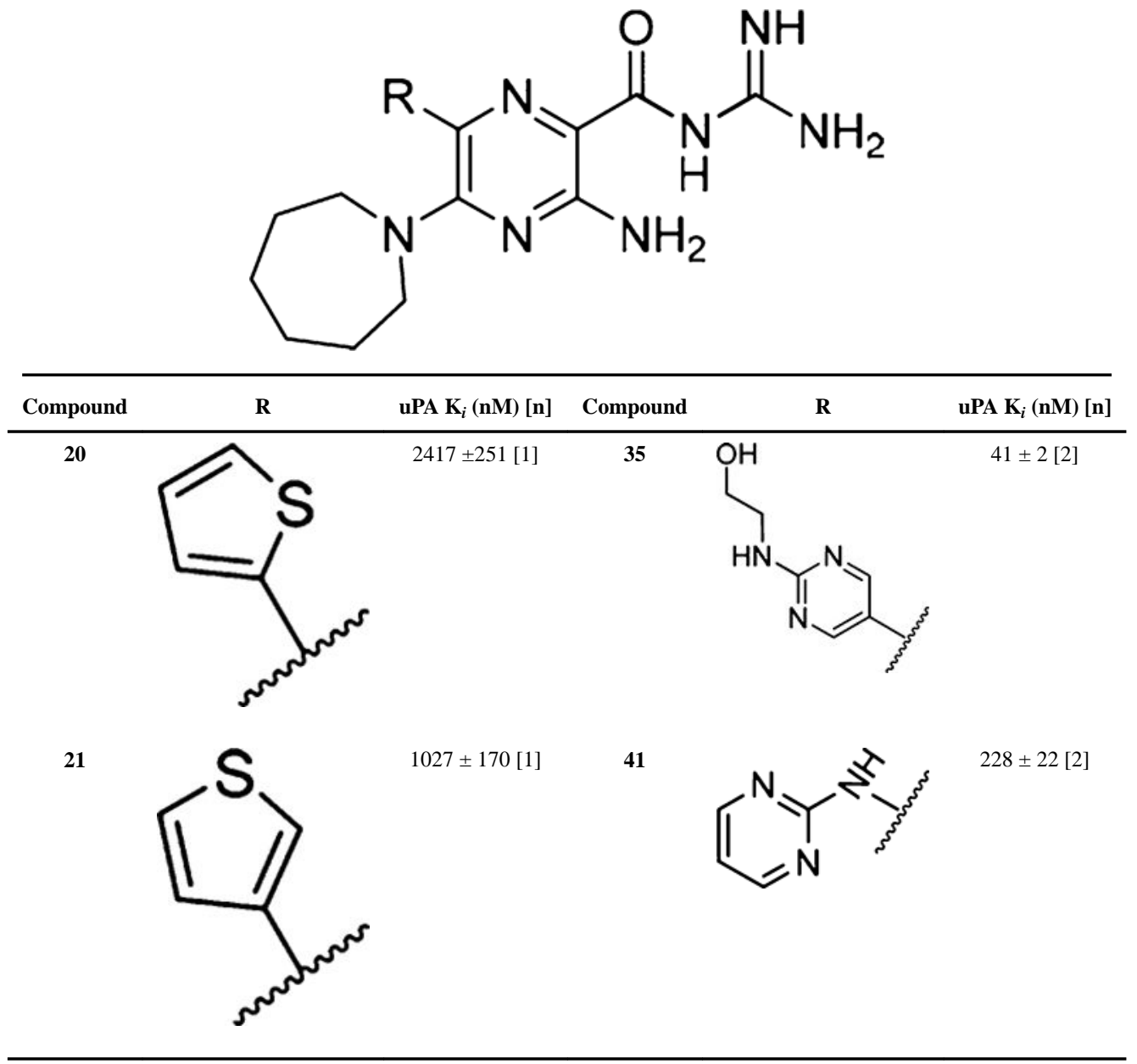

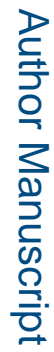

${ }^{a}$ For compounds where $n>1$, values represent the mean \pm SEM from identical repeat assays conducted on different days. $K_{\mathrm{i}}$ values were calculated from experimentally determined IC 50 values according to the methods of Cheng and Prussof. ${ }^{52}$ 


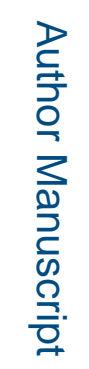

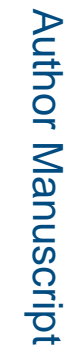

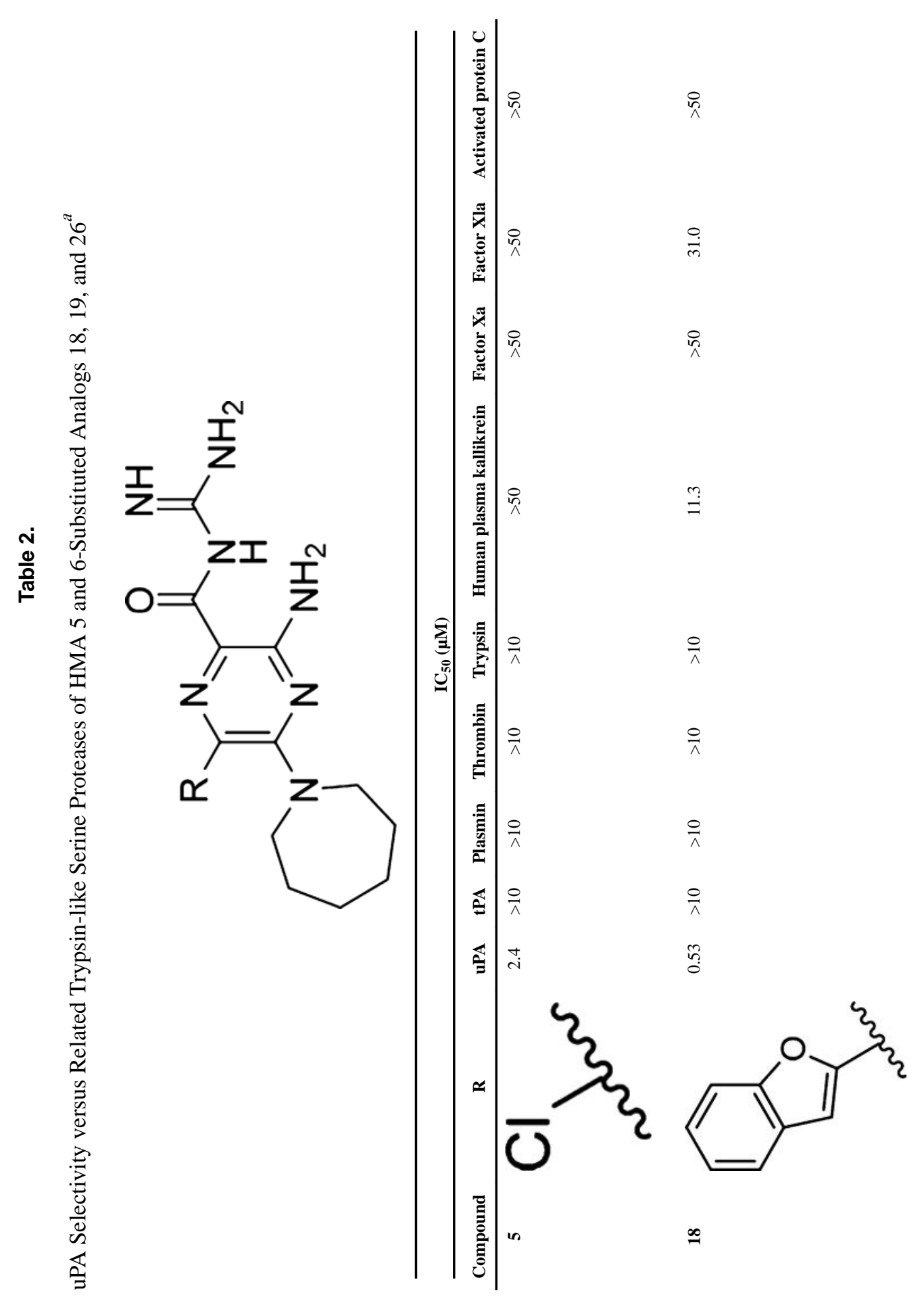

J Med Chem. Author manuscript; available in PMC 2019 September 27. 


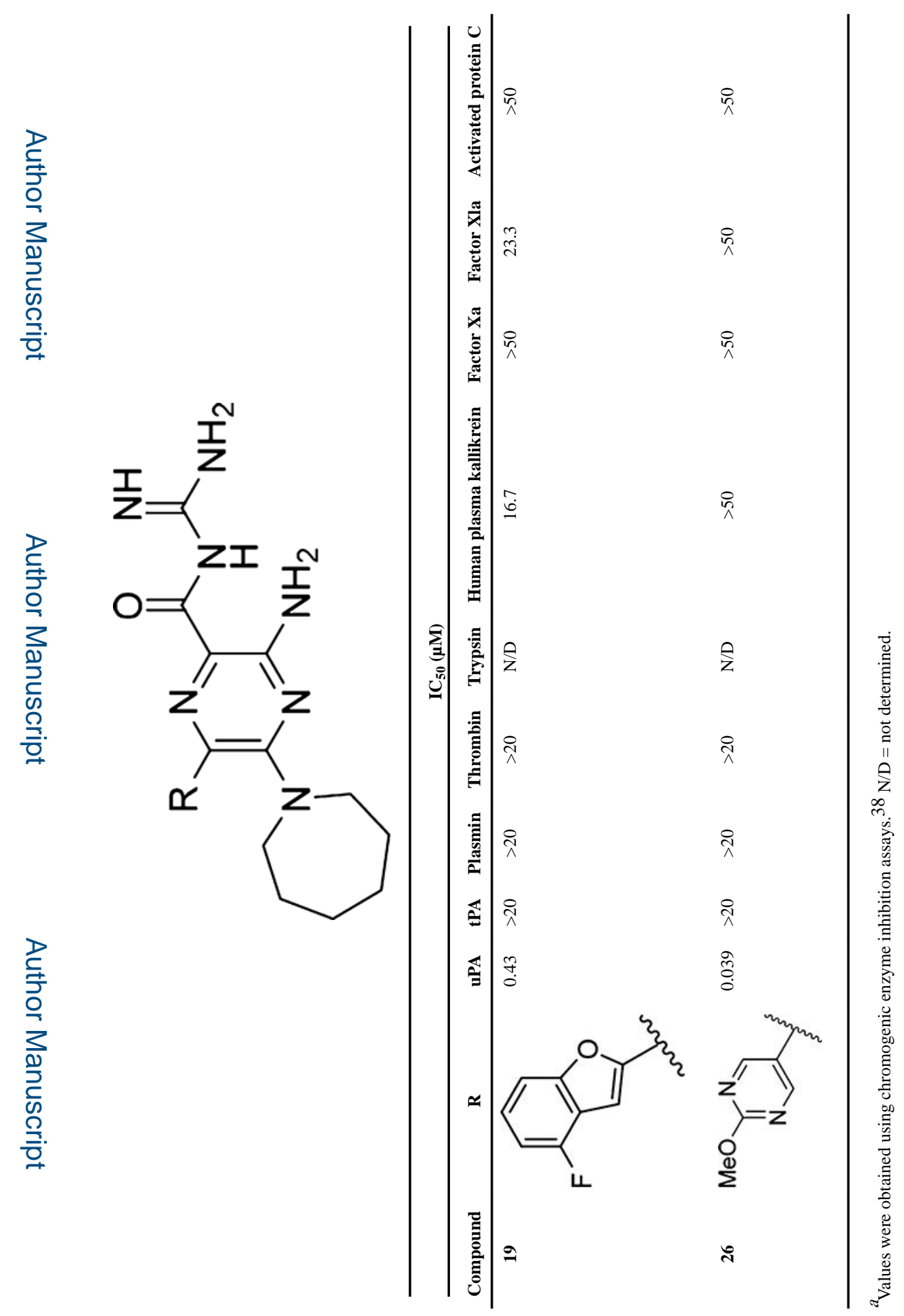

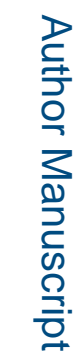


Table 3.

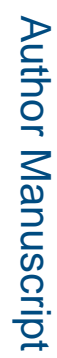

Effect of HMA and Selected Analogs on Human Cell Viability
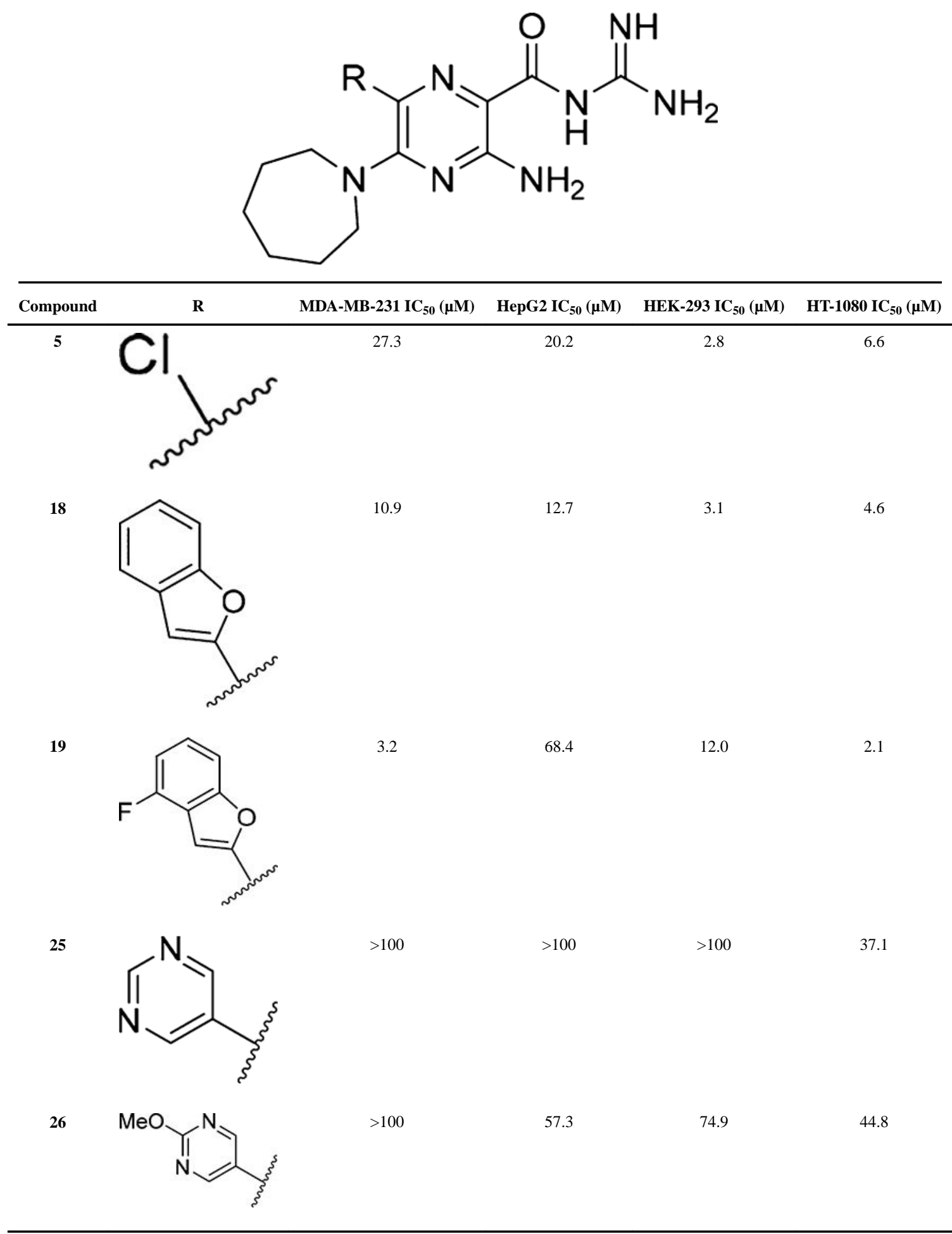

JMed Chem. Author manuscript; available in PMC 2019 September 27. 
Table 4.

Activities of Compounds 4, 5, 18, and 26 against Human and Mouse uPA

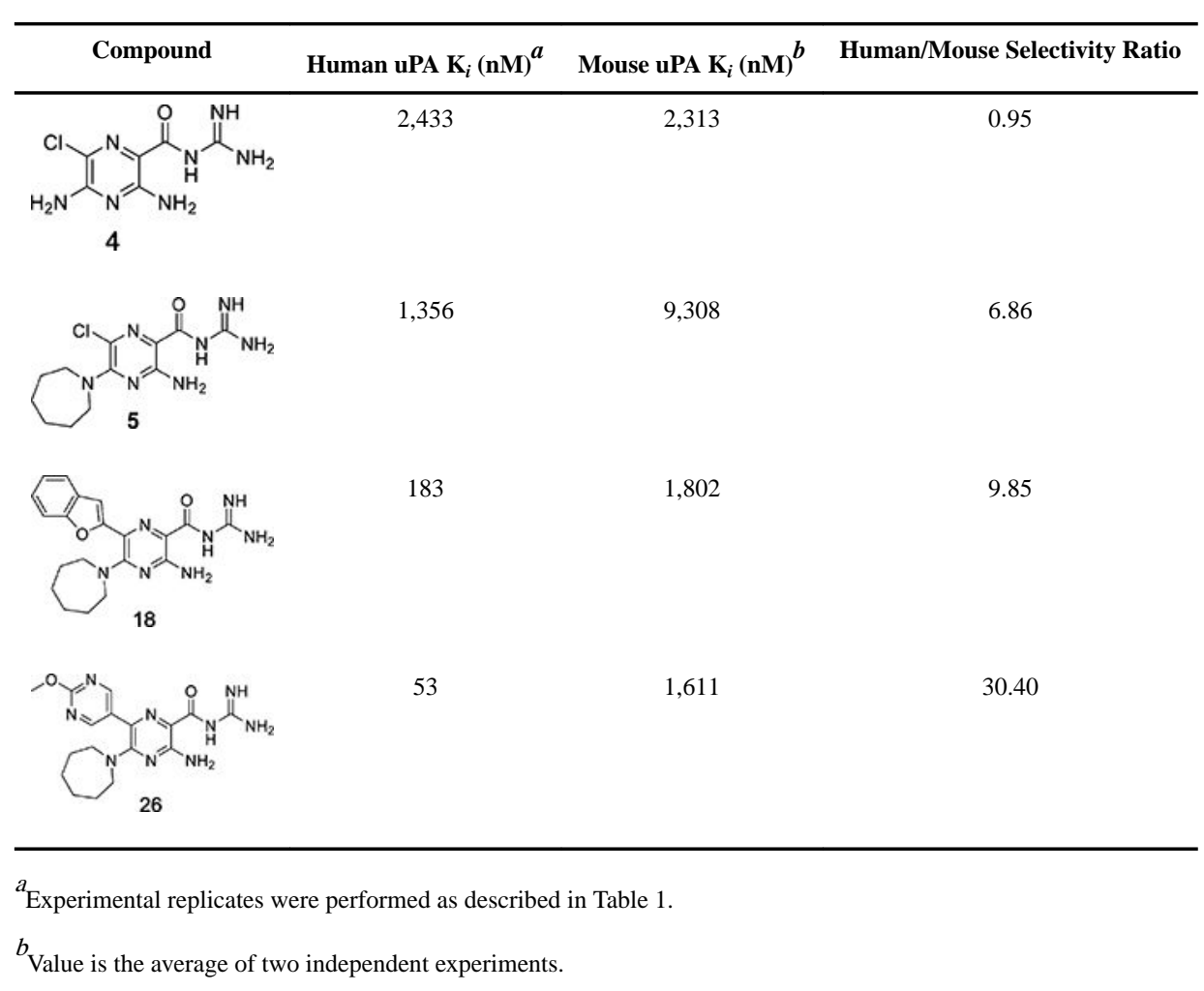

Experimental replicates were performed as described in Table 1

Value is the average of two independent experiments. 